\title{
ION/ION CHEMISTRY OF HIGH-MASS MULTIPLY CHARGED IONS
}

\section{Scott A. McLuckey, James L. Stephenson, Jr.}

Chemical and Analytical Sciences Division, Oak

Ridge National Laboratory, Oak Ridge, Tennessee

37831-6365

Received 1 October 1998; accepted 3 December 1998

I. Introduction

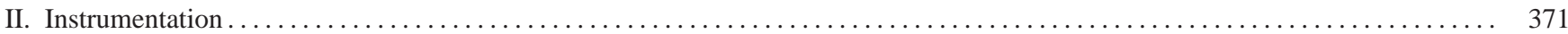

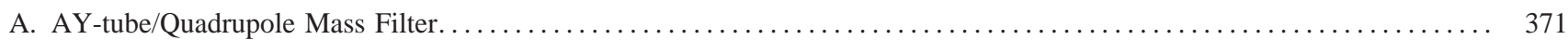

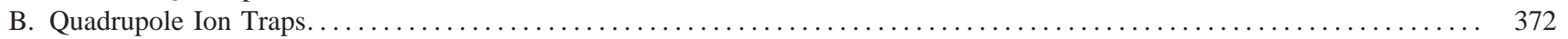

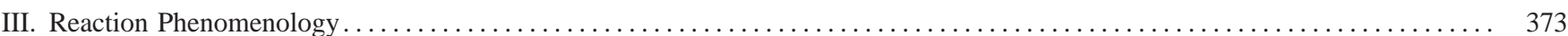

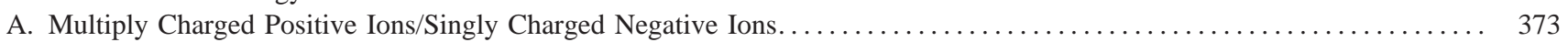

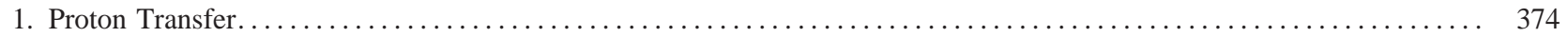

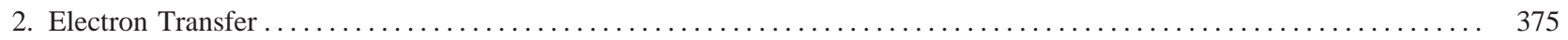

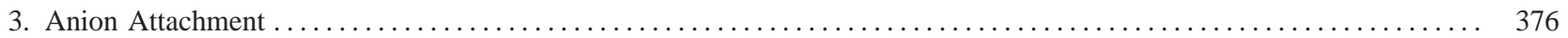

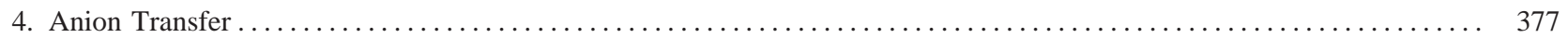

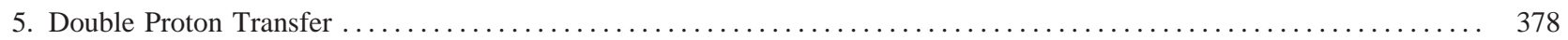

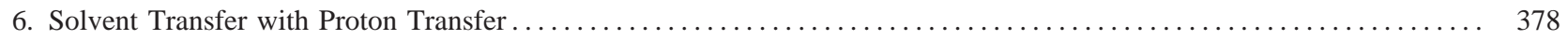

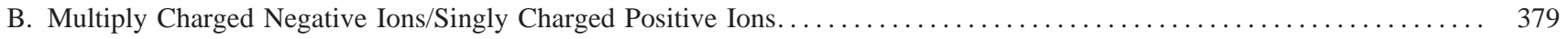

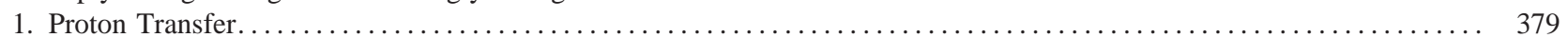

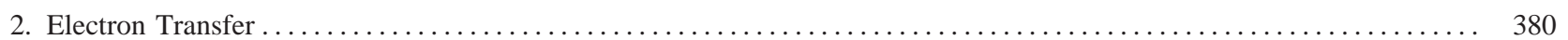

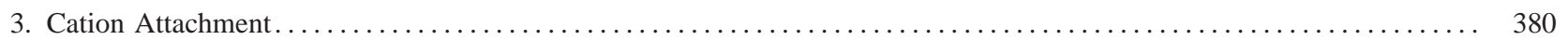

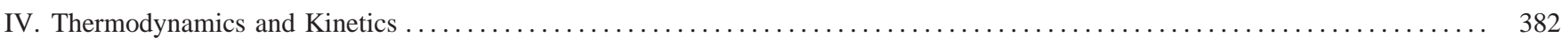

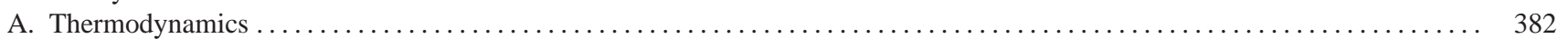

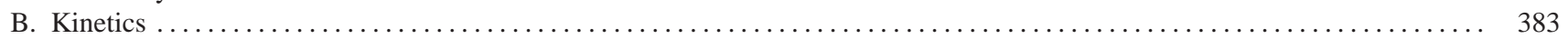

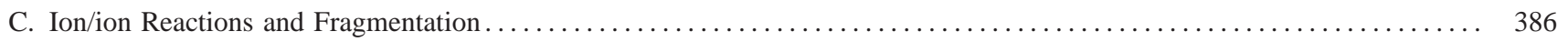

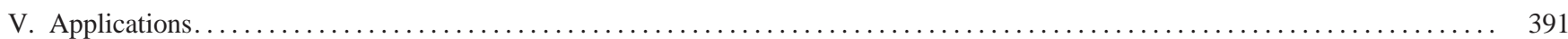

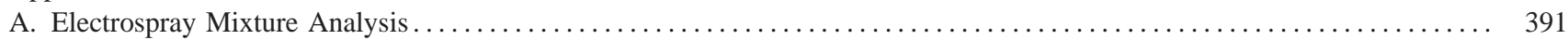

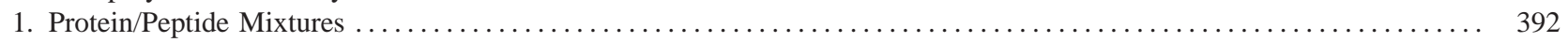

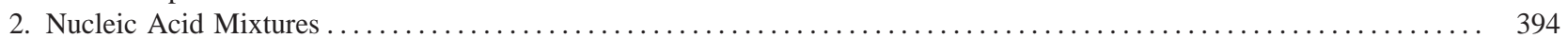

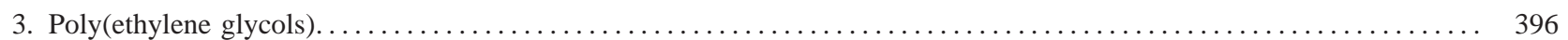

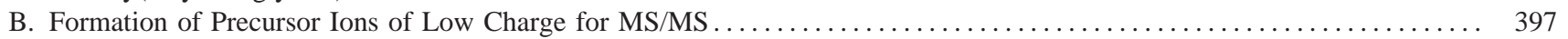

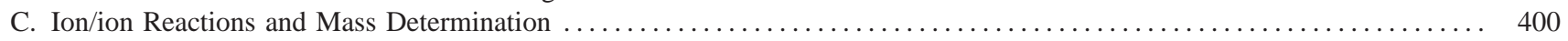

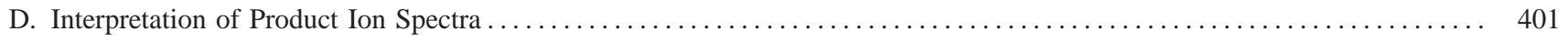

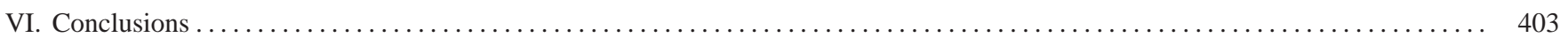

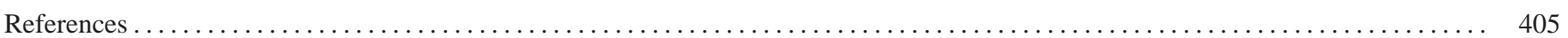

Electrospray ionization has enabled the establishment of a new area of ion chemistry research based on the study of the reactions of high-mass multiply charged ions with ions of opposite polarity. The multiple-charging phenomenon associated with electrospray makes possible the generation of multiply charged reactant ions

Correspondence to: Scott A. McLuckey

Contract grant sponsor: Division of Chemical Sciences, Office of Basic Energy Sciences, U.S. Department of Energy

Contract grant sponsor: National Institutes of Health that yield charged products as a result of partial neutralization due to ion/ion chemistry. The charged products can be readily studied with mass spectrometric methods, providing useful insights into reaction mechanisms. This review presents the research done in this area, all of which has been performed within the past decade.

Contract grant number: GM45372

Contract grant sponsor: Lockheed Martin Energy Research Corporation for the U.S. Department of Energy

Contract grant number: DC-AC05-96OR22464. 
Ion/ion chemistry has been studied at near-atmospheric pressure in a reaction region that leads to the atmospheric/vacuum interface of a mass spectrometer, and within a quadrupole ion trap operated with a bath gas at a pressure of 1 mtorr. Proton transfer has been the most common reaction type for high-mass ions, but other forms of "charge transfer," such as electron transfer and fluoride transfer, have also been observed. For some ion/ion reactions, attachment of the two reactants has been observed. Multiply charged ion/ion reactions are fast, due to the long-range Coulombic attraction, and they are universal in that any pair of oppositely charged ions is expected to react due to the high exothermicity associated with mutual neutralization. The kinetics of reaction for multiply charged ions, derived from the same molecule with a given singly charged reactant ion, follow a charge-squared dependence, at least under normal quadrupole ion trap conditions. This dependence suggests that reaction rates are determined by the long-range Coulomb attraction, and that the ions react with constant efficiency as a function of charge state. In the case of proton transfer reactions from polypeptides to even-electron perfluorocarbon anions, no fragmentation of the polypeptide product ions has, as yet, been observed. Electron transfer from small oligonucleotide anions to rare gas cations, on the other hand, results in extensive fragmentation of the nucleic acid product ions. The extent of fragmentation decreases as the size of the oligonucleotide anions increases, reflecting a decrease in fragmentation rates associated with an increase in the number of internal degrees of freedom of the oligonucleotide. When ion-cooling rates become competitive with dissociation rates, the initially formed product ions are stabilized and fragmentation is avoided. Collisional cooling, therefore, likely plays an important role in the relative lack of dissociation observed thus far as a result of ion/ion reactions for most high-mass ions. The observed dependence of ion/ion reaction rates on the square of the ion charge, the universal nature of mutual neutralization, and the relative lack of fragmentation that arises from ion/ion reactions, makes ion/ion chemistry a particularly useful means for manipulating charge states. This review emphasizes applications that take advantage of the unique characteristics of ion/ion proton transfer chemistry for manipulating charge states. These applications include mixture analysis by electrospray, precursor ion charge state manipulation for tandem mass spectrometry studies, and simplified interpretation of product ion spectra. (C) 1999 John Wiley \& Sons, Inc., Mass Spec Rev 17: 369-407, 1998

Keywords: electrospray; multiply charged ions; ion/ion reactions; ion chemistry

\section{INTRODUCTION}

The importance of the interactions of oppositely charged ions in the gas-phase was recognized over a century ago by Thomson and Rutherford (Thomson \& Rutherford, 1896), and the first experimental measurements designed to measure ion recombination rates were made by Rutherford shortly thereafter (Rutherford, 1897). Not surprisingly, those measurements and the bulk of almost all other studies of ion recombination since then have involved singly charged reactants. This consequence is due to the relevance of ion/ion reactions that involve singly charged ions to important areas of physical science (e.g., atmospheric chemistry, combustion, and plasma physics) and to the ease with which singly charged ions can be formed. The reader is referred to several reviews (Bates, 1985; Flannery, 1982a; Flannery, 1982b; Smith et al., 1976; Mahan, 1973) of ion/ion reactions that involve singly charged ions, wherein the discussion is largely devoted to reaction kinetics.

The advent of electrospray ionization (Fenn, et al., 1990; Smith, et al., 1990a; Cole, 1997; Gaskell, 1997) and its propensity to form multiply charged high-mass ions has made possible the study of the gas-phase chemistry of such species. By far, most attention has been, and continues to be devoted to the unimolecular (dissociation) chemistry of multiply charged ions derived from biopolymers, where dissociation is used principally to obtain primary structural information. (For a sampling of the large and growing literature on the dissociations of gaseous multiply charged biopolymers see Vékey, 1995; Price et al., 1996; Little et al., 1994; Senko, et al., 1994; Tang \& Boyd, 1992; McLuckey \& Vaidyanathan, 1997a.) Ion/molecule chemistry involving high- mass multiply charged ions has also become an active area of research. Heavy emphasis has been placed on proton transfer from multiply charged polypeptides to strong gaseous neutral bases (see, for example, McLuckey et al., 1990; Ogorzalek-Loo et al., 1992a; Ogorzalek-Loo \& Smith, 1995; Cassady et al., 1994; Williams, 1996; Green \& Lebrilla, 1997), and hydrogen/deuterium exchange reactions (Winger et al., 1992; Suckau et al.; 1993; Wood, et al., 1995; McLafferty et al., 1998). Covalent modification of gaseous oligonucleotides via nucleophilic substitution has also been reported (O'Hair \& McLuckey, 1997). Proton transfer and hydrogen/deuterium exchange reactions have been used to study fundamental properties of ions, such as their intrinsic basicities and gas-phase conformations. Ion/molecule proton transfer reactions have also been used as means for manipulating the charge states of high-mass ions for various purposes (McLuckey et al., 1991a; Smith et al., 1994; Hunter et al., 1994; McLuckey \& Goeringer, 1995a; Clemmer \& Jarrold, 1997; Ramsey \& McLuckey, 1997).

Although unimolecular and bimolecular ion/molecule chemistries of high-mass multiply charged ions will continue to play central and expanding roles in electrospray ionization research and applications, ion/ion chemistry that involves multiply charged high-mass ions with either singly charged or multiply charged ions of opposite polarity shows interesting promise as an area for development. Unlike ion/ion reactions involving two singly charged reactants, wherein all reaction products are neutral species, multiply charged ion/ ion reactions can yield charged products. This situation makes the study of multiply charged ion/ion chemistry far simpler than that of the ion/ion chemistry of singly charged ions, and allows for a much more direct means for determining mechanistic aspects of the reactions (e.g., proton transfer, electron transfer, anion transfer, etc.). The volume 
of work published in the area of multiply charged ion/ion chemistry is far smaller than either that for dissociation or for ion/molecule chemistry. Nevertheless, significant progress has been made in this area in recent years. This review summarizes the phenomenology that has been reported, highlights unique aspects of ion/ion reactions, and illustrates a number of applications of ion/ion chemistry, particularly as a tool for manipulating high-mass ion charge states.

\section{INSTRUMENTATION}

Published reports to date fall into one of two general sets of reaction conditions: (i) reactions that occur at near-atmospheric pressure prior to product ions (and unreacted ions) being sampled into the vacuum system of a mass spectrometer, and (ii) reactions that occur within the environment of a quadrupole ion trap operated with a bath gas comprised largely of room temperature helium at ca. 1 mtorr. Instrumentation associated with each set of reaction conditions is described in turn below. Although singly charged ion/electron recombination measurements have recently been reported (Malek \& Wanczek, 1996) and multiply charged ion/electron recombination has been described (Zubarev et al., 1998), both in the high-vacuum conditions of Fourier transform ion cyclotron resonance instruments, there are as yet no published reports of experiments involving multiply charged ion/ion reactions at pressures significantly below those reported in the quadrupole ion trap research.

\section{A. Y-tube/Quadrupole Mass Filter}

The first reports that described multiply charged ion/ion reactions (Ogorzalek-Loo et al., 1991; 1992b) involved merging ions of opposite polarity at near atmospheric pressure in a Y-tube arrangement prior to sampling ions into an atmosphere/vacuum interface. Ions of opposite polarity were introduced into separate arms of the Y-tube, where they merged in the flow that lead to the mass spectrometer inlet. A quadrupole mass filter was used to acquire the resulting mass spectra. A schematic diagram of the apparatus used in the later work is shown in Fig. 1 (Ogorzalek-Loo et al., 1992b).

The unique element in the apparatus of Fig. 1 is the Y-tube reactor. Glass and stainless steel versions of the Y-tube were both fabricated and used for ion/ion reaction studies. In the case of the glass Y-tube, each of the ends was made electrically conductive by depositing a silver coating such that the potential of each end could be defined independently. Droplet desolvation was effected by use of a counter-current flow of warm nitrogen and/or by heating the capillary. This Y-tube arrangement allowed for operation in three modes: (i) single electrospray (to record data in the absence of ion/ion reactions); (ii) dual electrospray, where

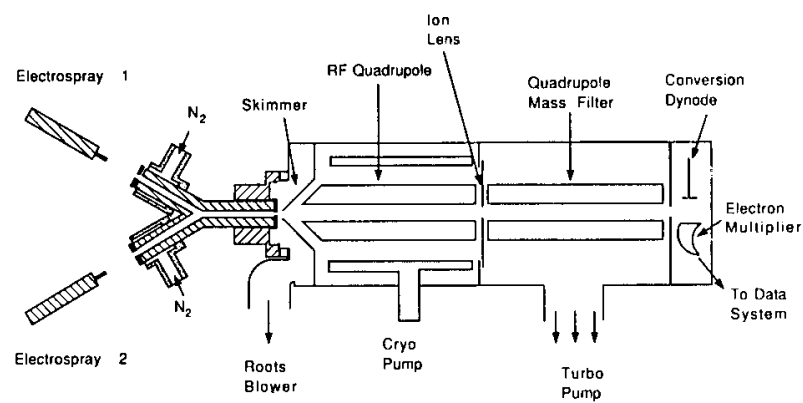

FIGURE 1. Instrument diagram of the apparatus that incorporates a Y-shaped capillary inlet/flow-reactor for ion/ion reaction studies. (Reprinted from J Am Soc.Mass Spectrom 1992, 3, 695-705, with permission from Elsevier Science, Inc. Copyright 1992 by the American Society for Mass Spectrometry.)

opposite ionization polarities were employed; and (iii) electrospray for multiply charged ion formation and atmospheric pressure discharge ionization for singly charged ion production. In the latter case, all three ion polarity combinations (viz., positive electrospray/negative discharge, negative electrospray/positive discharge, and positive electrospray/positive discharge) were studied.

The major advantages of the Y-tube apparatus for the study of ion/ion reactions are its relative simplicity and the ease with which it can be adapted to any kind of electrospray mass spectrometer. By carrying out the reactions external to the mass spectrometer, the characteristics of the mass analyzer itself place no limits on the kinds of ions that can be used as reactants. On the other hand, it was sometimes difficult to draw firm conclusions regarding the underlying factors that gave rise to observable changes in mass spectra acquired when both ion polarities were present vs. when only one ion source was being used. This complexity resulted from the limited ability to precisely define reaction conditions. A mixture of ions, solvent vapors, and atmospheric gases was typically present in the Y-tube. The mixture composition could vary according to ionization conditions and $\mathrm{Y}$-tube inlet aperture size. Furthermore, mass spectra were also often sensitive to the potential across the Y-tube outlet/skimmer region. In addition to uncertainties associated with the composition of the mixture of ions and neutral species in the reaction region, the limited mass-to-charge range of the quadrupole mass filter precluded the observation of high mass-to-charge products that were likely to be produced. For these reasons, some of the results described in the two published Y-tube studies raised a number of interesting questions that could not be answered with the apparatus of Fig. 1. Nevertheless, a variety of interesting and potentially useful phenomena were observed for the first time, including several chemical reactions that have yet to be observed under other reaction conditions (see below). 


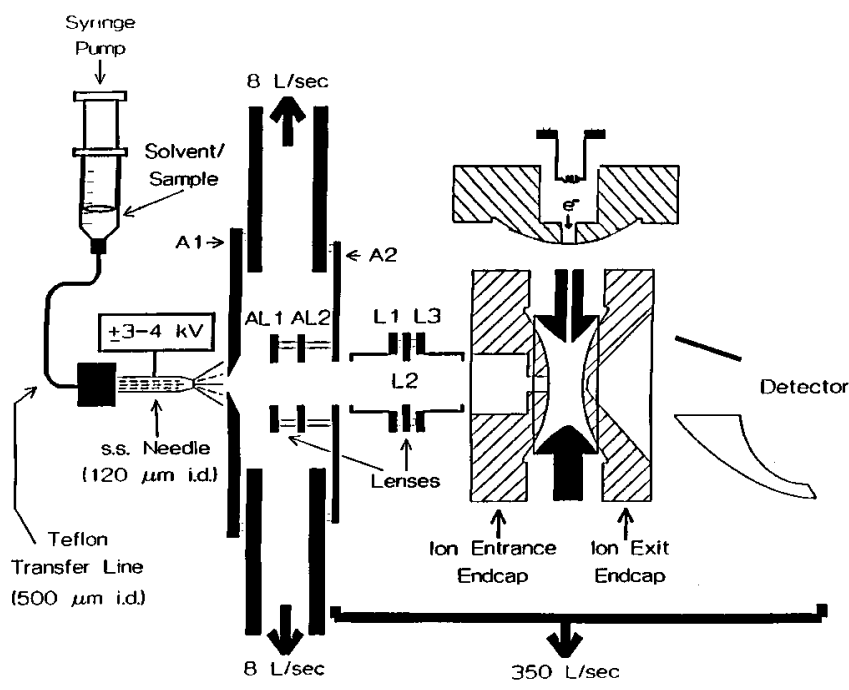

FIGURE 2. Side-view instrument schematic of a quadrupole ion trap that combines electrospray ionization and in situ ionization effected by injection of electrons through the ring-electrode.

\section{B. Quadrupole Ion Traps}

Recent work in our laboratory has taken advantage of the bipolar storage ability of the quadrupole ion trap (Mather \& Todd, 1980; Williams \& Cooks, 1993). This characteristic, and the fact that oppositely charged ion clouds overlap in space in the quadrupole ion trap environment, has facilitated the study of ion/ion chemistry in the dilute gas-phase. Furthermore, the "tandem-in-time" nature of the ion trap MS/MS and MS ${ }^{\mathrm{n}}$ experiments (Johnson et al., 1990; Louris et al., 1990; McLuckey et al., 1991b) is particularly advantageous in defining reaction conditions and in determining the genealogy of reaction products. Two approaches have thus far been used to form singly charged ions for reaction with multiply charged ions of opposite polarity, and each is described in turn below.

The first ion/ion reaction studies to be carried out in the quadrupole ion trap were conducted using an apparatus in which multiply charged negative ions were formed via electrospray and injected through an aperture in an end-cap electrode. Singly charged positive ions were formed within the ion trap via electron ionization of gaseous species admitted into the vacuum system (Herron et al., 1995a,b; Herron et al., 1996a, b; McLuckey et al., 1996a; McLuckey et al., 1997b). A side-view schematic diagram of this apparatus is shown in Fig. 2. Electron ionization was effected by drilling a 3-mm hole in the ring-electrode and mounting a filament to allow electrons to be injected into the ion trapping volume. Using this approach, positive ions formed directly via electron ionization could be used as reagents, or under the appropriate conditions for quadrupole ion trap chemical ionization (Creaser, 1995), protonated molecules could be generated to serve as the positive ion reagent. The typical experimental sequence involved (i) the introduction of multiply charged negative ions; (ii) an ion desolvation period (if necessary); (iii) an optional ion-isolation period; (iv) a short period in which electrons from the filament were gated into the ion trap; (v) a mutual storage period to allow ion/ion reactions to proceed; and (vi) mass analysis using resonance ejection (Kaiser et al., 1991). The major strength associated with this arrangement is the relative ease with which a source of positive ions (viz., positive ion electron ionization and positive ion chemical ionization) can be adapted to an electrospray/ion trap. However, the variety of species that can be formed in sufficient abundance to effect fast ion/ion reactions is limited to positive ions that can be derived from relatively volatile species. This arrangement is not well-suited to the study of multiply charged positive ions with singly charged negative ions, due to the inefficient nature of in situ negative ion formation using a heated filament (Berberich \& Yost, 1994).

All ion trap ion/ion reaction studies involving multiply charged positive ions and singly charged negative ions have been conducted with the apparatus shown schematically in Fig. 3 (Stephenson and McLuckey, 1997a). Multiply charged ions formed by electrospray are injected through an aperture in the entrance end-cap electrode, and singly charged ions formed in a glow discharge ionization source (McLuckey et al., 1988, Eckenrode et al., 1989) are injected through a 3-mm hole drilled in the side of the ring-electrode. The usual experimental sequence is similar to that for the electrospray/ heated filament ion trap described above, except that a period (typically $10-30 \mathrm{~ms}$ ) in which the glow discharge is initiated and maintained replaces the electron gate period mentioned above. The glow discharge source can be used to form either positive or negative ions, but the main motivation for coupling the glow discharge source to the ion trap was its ability to generate an intense beams of negative ions from species with relatively high cross-sections for electron capture (McLuckey et al., 1996b). Radial injection of ions into the ion trap (i.e., through a hole in the ring-electrode) is an inefficient process compared with axial ion injection (i.e., through a hole in an end-cap). The difference in ion injection efficiency is estimated to be about two orders of magnitude. Furthermore, the ions injected radially experience greater electric fields than those injected axially; that difference often leads to significantly greater degrees of fragmentation. The lower injection efficiency and higher propensity for fragmentation associated with radial injection combine to limit the range of singly charged ions that can be accumulated in high abundance within a few tens of milliseconds in the ion trap. Nevertheless, the apparatus of Fig. 3 has been useful in generating a variety of ion/ion combinations, and has allowed significant progress in understanding multiply charged ion/singly charged ion reaction chemistry in the dilute gas-phase.

The majority of multiply charged positive ion/singly charged negative ion studies in the ion trap to date have in- 


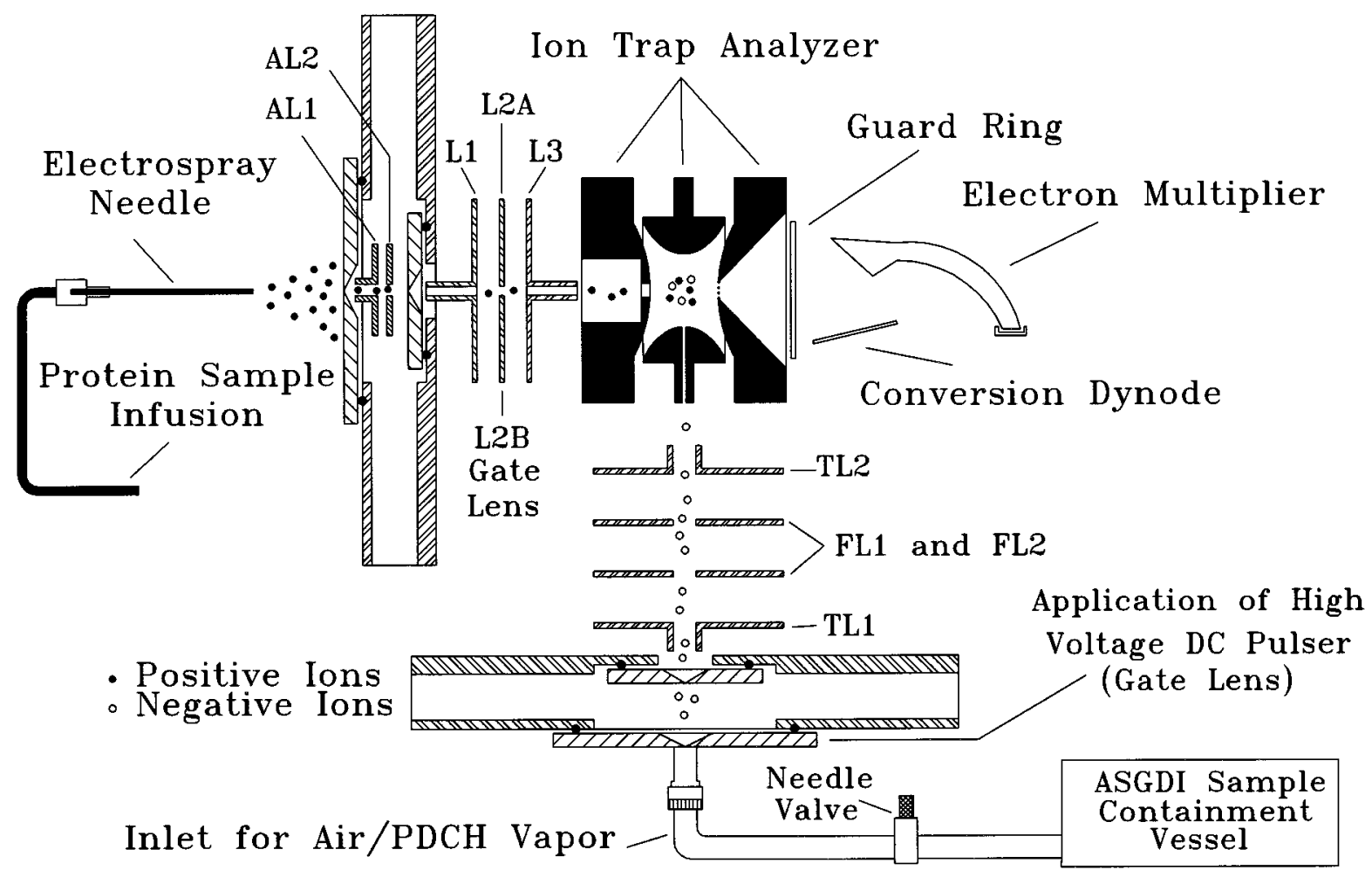

FIGURE 3. Side-view instrument schematic of a quadrupole ion trap that combines electrospray ionization (ions injected through an end-cap electrode) and glow discharge ionization (ions injected through the ringelectrode). (Reprinted from Int J Mass Spectrom Ion Proc 1997, 162, 89-106 with kind permission of Elsevier Science-NL, Sara Burgerhartstrasse 25, $1055 \mathrm{KV}$, Amsterdam, The Netherlands.)

volved negative ions formed from perfluorocarbons such as perfluoro-1,3-dimethylcyclohexane (PDCH). As elaborated upon further below, some of the negative ions derived from perfluorocarbons share several desirable characteristics for use in electrospray mixture analysis of species that form multiply charged ions. PDCH provides a good illustration of how strikingly different mass spectra can be using axial vs. radial injection. Figure 4 (Stephenson \& McLuckey, 1997a) compares mass spectra derived from the glow discharge ionization of PDCH using axial injection [Fig. 4(a)] vs. radial injection [Fig. 4(b)]. Ion accumulation times ca. $100 \times$ longer are required for radial injection than for axial injection to provide comparable total ion abundances in the mass spectra. Figure 4(a) shows that the molecular anion of PDCH dominates in the mass spectrum when the ions are injected axially, whereas Figure 4(b) shows that very few molecular anions survive the radial ion injection/ capture process. The negative ions of Fig. 4(b), or a subset thereof, comprise the anions from which much of the positive multiply charged ion/singly charged negative ion reaction data in the ion trap have been collected.

\section{REACTION PHENOMENOLOGY}

In singly charged ion/ion reaction studies, the mechanism for mutual neutralization is usually assumed (or not addressed), because the products are not identified. Obviously, an advantage of ion/ion reactions with multiply charged ions is that at least one of the reaction products retains charge, thereby allowing for its characterization by mass spectrometric approaches. Despite the relatively few combinations of positive ions and negative ions that have thus far been studied, a remarkably wide range of reaction phenomenologies has been noted. This section summarizes the generic reaction types with selected illustrative examples for multiply charged positive ions/singly charged negative ions and multiply charged negative ions/singly charged positive ions.

\section{A. Multiply Charged Positive Ions/Singly Charged Negative Ions}

Virtually all the multiply charged positive ions studied thus far have been comprised of a relatively high-mass polymer, such as a protein or a commercial polymer, with an excess of cations. The generic multiply charged positive ion is represented herein as $(\mathrm{M}+\mathrm{nX})^{\mathrm{n}+}$, where $\mathrm{X}$ is either $\mathrm{H}^{+}$or $\mathrm{Na}^{+}$, with the proton being by far most common. Anions are represented generically as $\mathrm{Y}^{-}$for even-electron species and $\mathrm{Y}^{-}$for odd-electron species. For cases in which part of the 


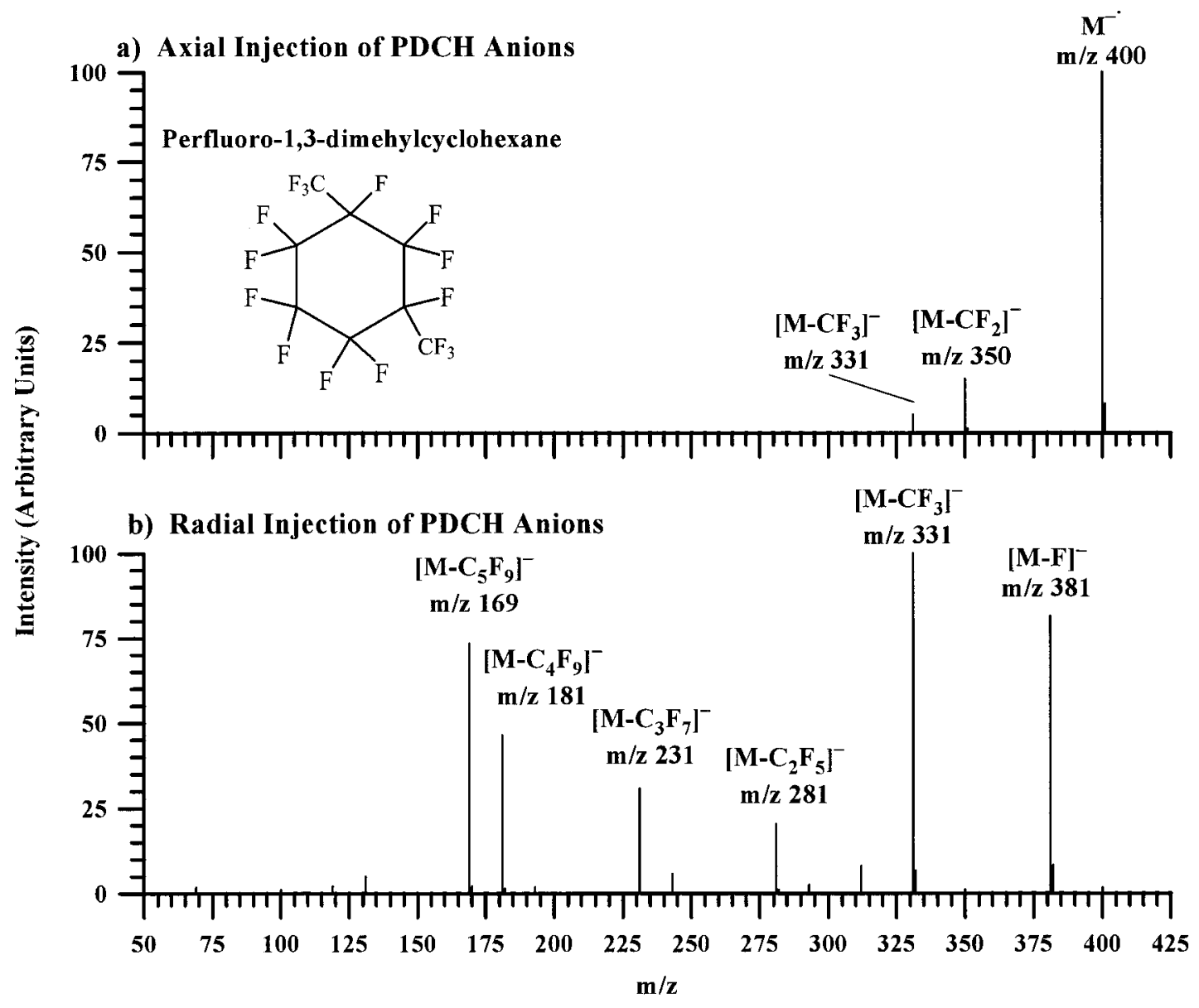

FIGURE 4. (a) Air/PDCH glow discharge mass spectrum obtained from axial injection of ions into a quadrupole ion trap. (b) Air/PDCH glow discharge mass spectrum obtained from radial injection of ions into a quadrupole ion trap. (Reprinted from Int J Mass Spectrom Ion Proc 1997, 162, 89-106 with kind permission of Elsevier Science-NL, Sara Burgerhartstrasse 25, 1055 KV, Amsterdam, The Netherlands.)

negative ion is transferred to the positive ion, the negative ion is represented as $\mathrm{YZ}^{-}$, where $\mathrm{Z}$ is the transferred fragment.

\section{Proton Transfer}

Proton transfer is, by far, the most commonly observed reaction for multiply protonated polypeptides. This reaction dominates, because all the ion trap studies focused on multiply protonated bioploymers in which even-electron perfluorocarbon anions served as the negative ion reactants (see, for example, Stephenson \& McLuckey, 1996a, b; Stephenson et al., 1997a-e; Stephenson \& McLuckey, 1998a-c). Proton transfer was probably a major reaction that took place in the Y-tube discharge studies as well (Ogorzalek-Loo et al., 1992b), although the extent to which proton transfer in conjunction with solvent transfer contributed to the charge state reduction in the Y-tube studies is unclear (see below). In the ion trap studies, it was known that unsolvated anions were the reactants.
The transfer of a proton to a singly charged negative ion is represented as:

$$
(\mathrm{M}+n \mathrm{H})^{n+}+\mathrm{Y}^{-} \rightarrow(\mathrm{M}+(n-1) \mathrm{H})^{(n-1)+}+\mathrm{HY} .
$$

The neutral product is neither analyzed nor detected. Although it is shown here as the conjugate acid of the negative ion reactant, fragmentation of the neutral product cannot be precluded. Fragmentation of the ionic product that results from proton transfer, on the other hand, would be apparent if it occurred to a measurable extent. No clear evidence has thus far been apparent in any published ion/ion studies of dissociative proton transfer whereby a high-mass positive ion dissociates as a result of proton transfer to a negative ion (see Section IV). Figure 5 provides an illustrative example of typical data that result from an ion trap ion/ion reaction experiment involving a small- to moderate-size multiply protonated protein (bovine ubiquitin) and the (M-F $\cdot)^{-}(\mathrm{m} / \mathrm{z}$ 381) and $\left(\mathrm{M}^{\left.-\mathrm{CF}_{3}\right)^{-}}{ }^{-}(\mathrm{m} / \mathrm{z}, 331)\right.$ negative ions derived from 

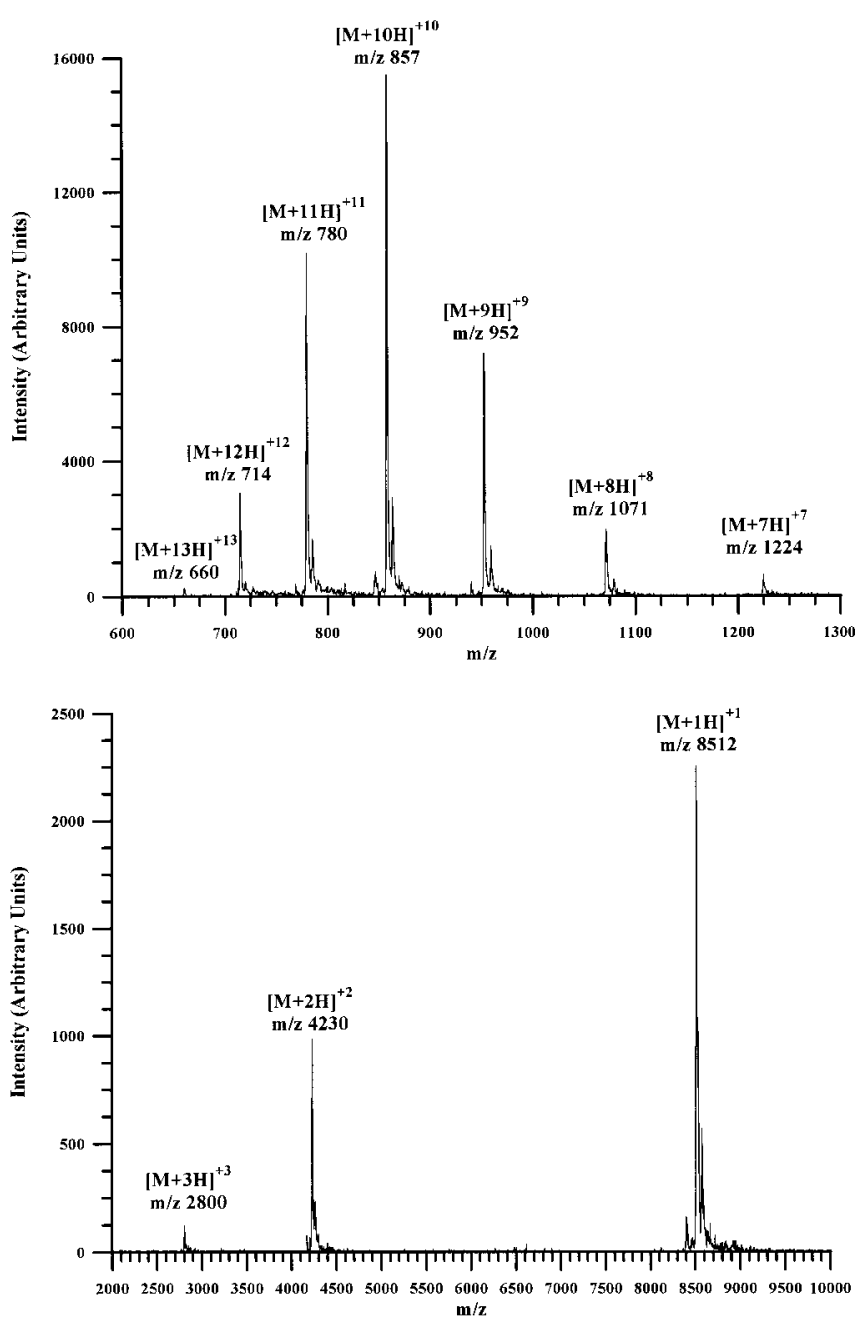

FIGURE 5. Electrospray mass spectrum of bovine ubiquitin (a) before admission of negative ions and (b) after admission of negative ions derived from PDCH and a mutual storage time of $305 \mathrm{~ms}$. (Reprinted with permission from J Am Chem Soc 1996, 118, 7390-7397, copyright 1996 American Chemical Society.)

PDCH [see Fig. 4(b)] (Stephenson \& McLuckey, 1996a). Figure 5(a) shows the normal electrospray mass spectru$\mathrm{m}$, and Fig. 5(b) shows the mass spectrum acquired after the ubiquitin ions of 5(a) were subjected to reaction with the PDCH anions for $330 \mathrm{~ms}$. (Similar results have been obtained with reaction times of ca. $100 \mathrm{~ms}$ as a result of optimization of the alignment of the discharge source with the hole in the ring-electrode to significantly improve anion accumulation performance.)

As with all multiply charged species thus far studied with the ion trap instruments, where the singly charged high-mass ion could be stored and analyzed, the ubiquitin ions can be reduced to unit charge (and completely neutralized at sufficiently long reaction times). As discussed in the following section, there appears to be no inherent limitation to neutralization. Ion/ion chemistry is a very effective means to reduce the charge of a multiply charged ion. Note also the absence of fragments in the spectrum of Fig. 5(b). Despite the fact that the ions that eventually become singly charged have undergone 6-12 consecutive highly exothermic proton transfer reactions, no measurable fragmentation is observed (see Section IV.C). By extension of the ion trap results, which were obtained using a bath gas pressure of about 1 mtorr, it is hardly surprising that no evidence was noted for fragmentation as a result of ion/ion reactions in the Y-tube studies conducted at near atmospheric pressure.

The signal levels in Fig. 5(b) are significantly lower than those of Figure 5(a), although there is little or no decrease in signal-to-noise ratio. The decrease in signal can arise from a number of possible factors, which include (i) differences in detector efficiency as a function of charge state (Axelsson et al., 1994; Loo \& Pesch, 1994); (ii) total neutralization; (iii) difference in effective scan speed for the two experiments; and (iv) ion losses that result from differences in ion storage efficiency as the $m / z$ ratio increases. Of these factors, difference in detector efficiency and scan speed are believed to play the most important roles in determining the differences in signal levels in the comparison of Fig. 5. For ions with $\mathrm{m} / \mathrm{z}$ values greater than about $3 \times 10^{4}$, ion storage losses are expected to become significant in the ion trap instruments described in Section II (Stephenson \& McLuckey, 1997a).

\section{Electron Transfer}

An electron transfer reaction that involves a multiply charged positive ion and a singly charged negative ion is indicated generically as:

$$
(\mathrm{M}+n \mathrm{X})^{n+}+\mathrm{Y}^{-\bullet} \rightarrow(\mathrm{M}+n \mathrm{X})^{(n-1)+\bullet}+\mathrm{Y}
$$

Reaction (2) indicates the transfer of an electron from the negative ion to the multiply charged positive ion. In the usual case, electrospray forms even-electron, closed-shell reactant ions; those reactions imply that the product ion is an oddelectron hypervalent species. Such species, derived from multiply protonated proteins via electron capture, have recently been reported (Zubarev, 1998). In contrast with electron capture, however, electron transfer must compete with cation transfer (usually proton transfer), as indicated in reaction (3):

$$
(\mathrm{M}+n \mathrm{X})^{n+}+\mathrm{Y}^{-\cdot} \rightarrow(\mathrm{M}+(n-1) \mathrm{X})^{(n-1)+}+\mathrm{XY}^{\bullet}
$$

Reaction (3) differs from reaction (1) in that the negative ion is an odd-electron species rather than an even-electron species. Electron transfer is expected to be more competitive with cation transfer if the negative ion is an odd-electron species, because it does not require electron unpairing as does electron transfer between even-electron species. Nev- 


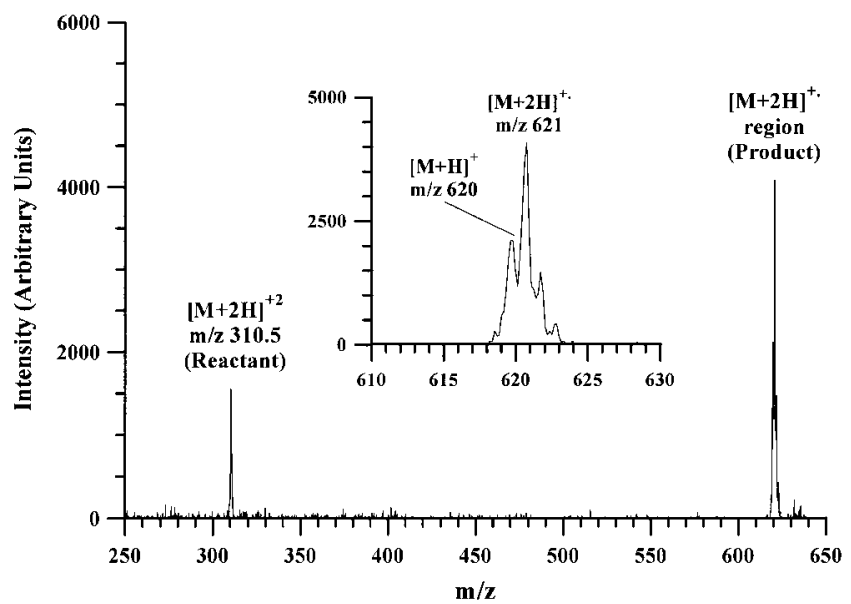

FIGURE 6. Post-ion/ion reaction spectrum obtained after doubly protonated meso- tetra(4-pyridyl) porphine was exposed to a population of negative ions formed from a sulfur dioxide glow discharge. The inset shows an expanded view of the product ion region with the signal at $\mathrm{m} / \mathrm{z} 621$ corresponding to the $(\mathrm{M}+2 \mathrm{H})^{+\bullet}$ ion. (Reprinted from Int J Mass Spectrom Ion Proc 1997, 162, 89-106 with kind permission of Elsevier Science- NL, Sara Burgerhartstrasse 25, $1055 \mathrm{KV}$, Amsterdam, The Netherlands.)

ertheless, proton transfer [reaction (3)] has been observed as the dominant reaction mechanism for most multiply charged positive ions, including the polypeptide ions that have been subjected to reaction with the limited set of odd-electron anions thus far studied.

Electron transfer [reaction (2)], however, has been observed with cations that are highly conjugated. Figure 6 , for example, shows the results of an ion/ion reaction experiment between mass-selected doubly protonated meso-tetra(4-pyridyl) porphine and negative ions that derived from a sulfur dioxide discharge (Stephenson \& McLuckey, 1997a). The major negative ions in the mixture of ions allowed to react with the dication were, in order of abundance, $\mathrm{SO}_{2}^{-}, \mathrm{SO}_{3}^{-}$, and $\mathrm{O}_{2}^{-\bullet}$. The inset shows that reaction types (2) and (3) both gave rise to products [viz., $(\mathrm{M}+2 \mathrm{H})^{+\bullet}$ and $(\mathrm{M}+\mathrm{H})^{+}$, respectively]. It was not determined whether each negative ion reacted exclusively by either reaction (2) or reaction (3), or whether one or more of the negative ions showed any competition between the two charge transfer mechanisms. Note that there is essentially no evidence for fragmentation in Fig. 6; that lack of fragmentation indicates that neither charge transfer mechanism resulted in sufficient product ion excitation to lead to dissociation under normal ion trap storage conditions.

\section{Anion Attachment}

Anion attachment involves a two-body to one-body reaction, in which the energy of condensation must be dissipated to avoid fragmentation. The reaction can be written generically as:

$$
(\mathrm{M}+n \mathrm{X})^{n+}+\mathrm{Y}^{-} \rightarrow(\mathrm{M}+n \mathrm{X}+\mathrm{Y})^{(n-1)+}
$$

Despite the high exothermicity associated with ion/ion reactions, we have found that some anions have a high propensity for attachment to multiply charged positive ions without leading to fragmentation in the ion trap environment (Stephenson \& McLuckey, 1997e; Stephenson \& McLuckey, 1998d). Iodide $\left(\mathrm{I}^{-}\right)$has been found to show the greatest propensity for attachment to multiply charged cations. Figure 7 , for example, shows the ion/ion reaction spectrum of the +9 charge state of ubiquitin after being stored with a population of $\mathrm{I}^{-}$for about $25 \mathrm{~ms}$ (Stephenson \& McLuckey,

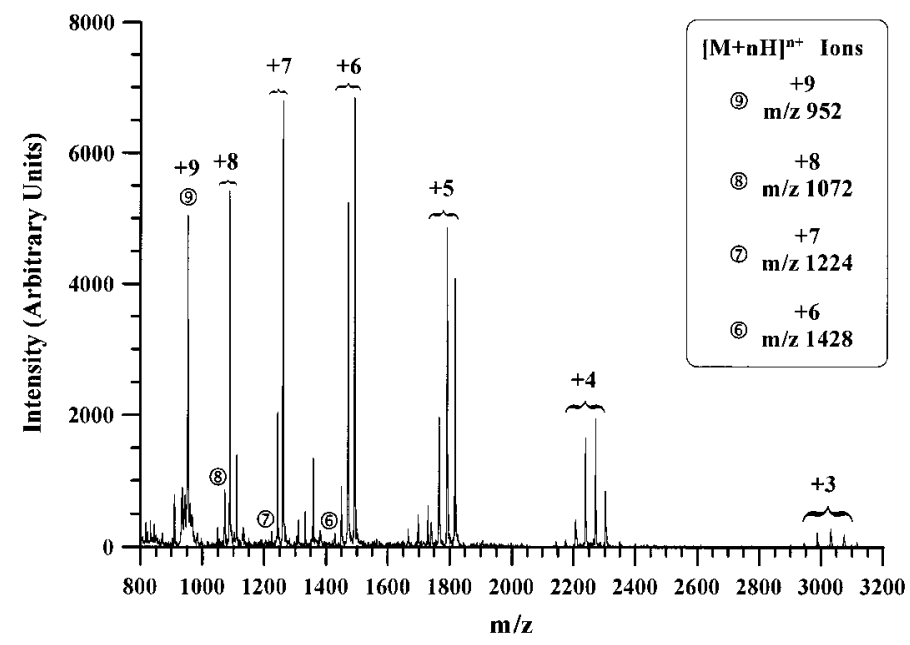

FIGURE 7. Post-ion/ion reaction spectrum obtained after the $(\mathrm{M}+9 \mathrm{H})^{9+}$ ion of bovine ubiquitin was exposed to a populations of $\mathrm{I}^{-}$for $25 \mathrm{~ms}$. The circled numbers indicate the $(\mathrm{M}+\mathrm{nH})^{\mathrm{n}+}$ ions with no $\mathrm{I}^{-}$attachment. (Reprinted with permission from J Am Chem Soc 1997, 119, 1688-1696, copyright 1997 American Chemical Society.) 
1997e). Note that most of the +9 charge state yields +8 products with a single $\mathrm{I}^{-}$attached, the +7 product distribution shows the attachment of two iodide anions, and the +6 product distribution shows the attachment of three iodide anions as the most abundant product. All the product charge states, however, show a distribution of products with varying numbers of $\mathrm{I}^{-}$ions attached. Further studies showed that the attachment species were intermediates to proton transfer to $\mathrm{I}^{-}$, wherein the initially formed intermediate was either cooled and trapped as the attachment product, or went on to give the proton transfer products anticipated by reaction (1). That is, in the first step of the reaction sequence, the +9 precursor ion yielded either the attachment product or the proton transfer product; i.e.,

$$
\begin{aligned}
&(\mathrm{M}+9 \mathrm{H})^{9+}+\mathrm{I}^{-} \rightarrow\left(\mathrm{M}+9 \mathrm{H}+\mathrm{I}^{-}\right)^{8+*} \rightarrow(\mathrm{M}+8 \mathrm{H})^{8+}+\mathrm{HI} \\
&-\mathrm{He} \rightarrow\left(\mathrm{M}+9 \mathrm{H}+\mathrm{I}^{-}\right)^{8+}
\end{aligned}
$$

where a third body $(\mathrm{He})$ is indicated as participating in the formation of the stabilized adduct. The two +8 products proceeded to react either by proton transfer or attachment, and so on.

The perfluorocarbon anions that react exclusively by proton transfer, such as those derived from $\mathrm{PDCH}$, also presumably proceed through a long-lived intermediate; however, such an intermediate has yet to be observed. In fact, the perfluorocarbon anions and ${ }^{\mathrm{I}-}$ comprise the two extreme cases for predominant proton transfer vs. predominant attachment. Most other negative ions show intermediate behavior when it comes to the competition between reaction (1) and reaction (4). The key underlying factor appears to be the lifetime of the intermediate, which, in turn, is largely determined by the binding strength of the conjugate acid of the negative ion with the cation attachment site. In the case of $\mathrm{I}^{-}$and multiply protonated polypeptides, it is the binding strength of hydroiodic acid with the various possible basic sites that largely determines the likelihood for attachment. Based on the ion/ion reaction observations, it was predicted and demonstrated that HI should attach to polypeptide cations with available neutral basic sites, and that the maximum number of HI molecules that attach usually equals the sum of the number of arginines, lysines, histidines, and N-termini, less the charge of the ion (Stephenson \& McLuckey, 1997e).

\section{Anion Transfer}

If the category of "charge transfer" is defined more broadly than being simply synonymous with "electron transfer," then cation and anion transfer are two other members of this group. Proton transfer has been a commonly observed
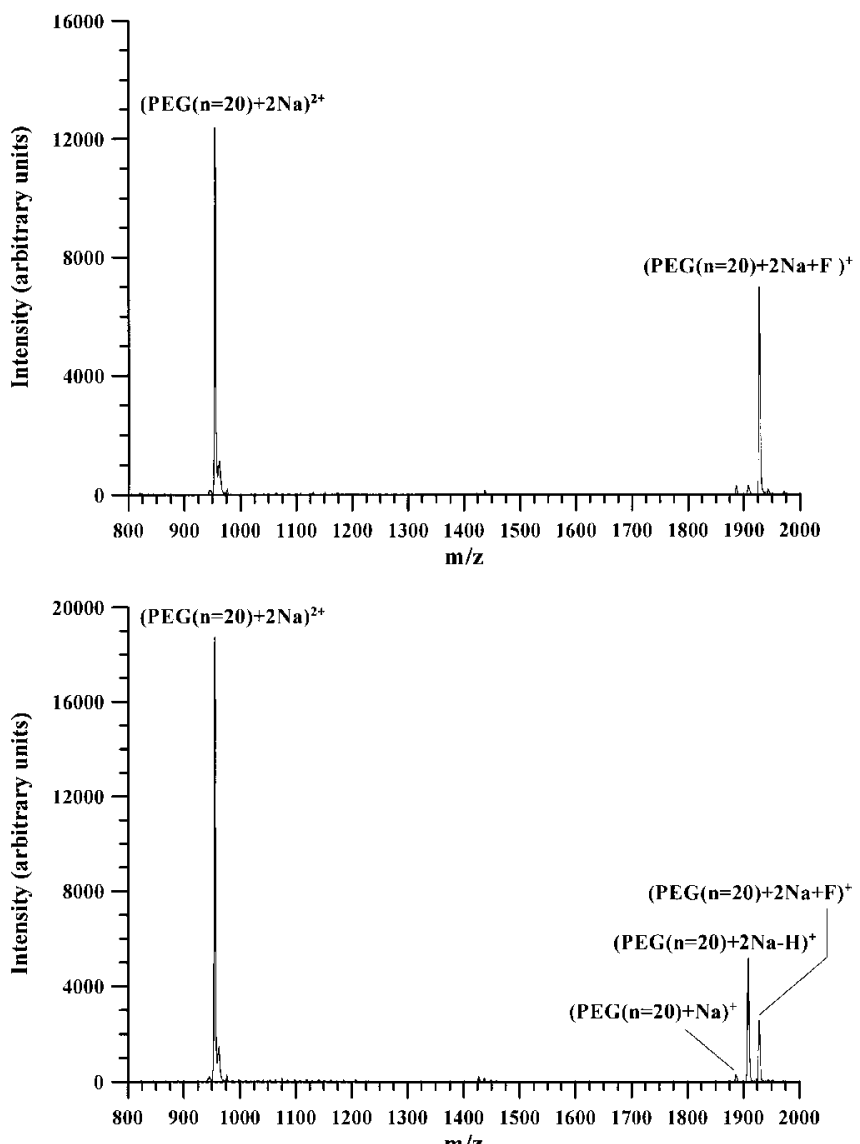

FIGURE 8. Post-ion/ion reaction spectra of the $(\mathrm{M}+2 \mathrm{Na})^{2+}$ of $\mathrm{PEG}_{20}$ exposed to (a) the $\left(\mathrm{M}-\mathrm{F}^{*}\right)^{-}$and $\left(\mathrm{M}-\mathrm{CF}_{3} .\right)^{-}$anions from $\mathrm{PDCH}$ and $(\mathrm{b})$ the $\left(\mathrm{M}-\mathrm{F}^{*}\right)^{-},(\mathrm{M}-\mathrm{CF} 3)^{-},\left(\mathrm{M}-\mathrm{C}_{4} \mathrm{~F}_{9 .}\right)^{-}$, and $\left(\mathrm{M}-\mathrm{C}_{5} \mathrm{~F}_{9 .}\right)^{-}$anions from PDCH. (Reprinted from the J Am Soc Mass Spectrom 1998, 9, 957-965, with permission from Elsevier Science, Inc. Copyright 1998 by the American Society for Mass Spectrometry.)

process, as described above, but anion transfer has also been observed (Stephenson \& McLuckey, 1998d). This reaction is written generically as:

$$
(\mathrm{M}+n \mathrm{X})^{n+}+\mathrm{YZ}^{-} \rightarrow(\mathrm{M}+n \mathrm{X}+\mathrm{Z})^{(n-1)+}+\mathrm{Y}
$$

The only examples in which anion transfer has been observed have involved the transfer of fluoride $\left(\mathrm{F}^{-}\right)$to positive ions that are not expected to readily undergo proton transfer. The most dramatic example observed thus far is illustrated in Fig. 8. Figure 8(a) shows the MS/MS spectrum of doubly charged poly(ethylene glycol) $20\left(\mathrm{PEG}_{20}\right)$ after reaction with the $\left(\mathrm{M}-\mathrm{F}^{\circ}\right)^{-}$and $\left(\mathrm{M}-\mathrm{CF}_{3}^{*}\right)^{-}$ions derived from $\mathrm{PDCH}$. The di-cation in this case, formally the $(\mathrm{M}+2 \mathrm{Na})^{2+}$ ion, reflects the usual observation that electrospray of PEGs results in cationization primarily by sodium ions (Wong et al., 1988). With the benefit of MS/MS, it was found that the high-mass perfluorocarbon anions from $\mathrm{PDCH}$, as well as the $\left(\mathrm{M}-\mathrm{F}^{*}\right)^{-}$ ion from perfluoro(methyldecalin), react with multiply 
charged PEG cations by fluoride transfer. It was also found that the $\left(\mathrm{M}-\mathrm{C}_{4} \mathrm{~F}_{9}^{*}\right)^{-}$and $\left(\mathrm{M}-\mathrm{C}_{5} \mathrm{~F}_{9}^{\circ}\right)^{-}$anions derived from $\mathrm{PDCH}$ reacted by removing a proton from PEG $(\mathrm{M}+\mathrm{nNa})^{\mathrm{n}+}$ cations. Interestingly, no perfluorocarbon anions were found to react to an appreciable extent by sodium ion transfer. When the mixture of PDCH anions indicated in Fig. 4(b) was allowed to react with PEG polycations, the mixture of resulting products reflected the contributions from fluoride and proton transfer. Figure 8(b) demonstrates this phenomenology with the MS/MS spectrum of the $\mathrm{PEG}_{20}$ di-cation after reaction with a mixture of low-mass and high-mass anions derived from PDCH.

\section{Double Proton Transfer}

The first Y-tube report principally described a reaction in which deprotonated molecules [viz., fluorescein and adenosine 5'-monophosphate (ATP)] formed by electrospray were reacted with multiply protonated polypeptides (viz., melittin, myoglobin, and cytochrome $c$ ) to produce the protonated fluorescein or ATP in the positive ion mass spectrum. This reaction, which was referred to as charge inversion, is written generically as:

$$
(\mathrm{M}+n \mathrm{H})^{n+}+\mathrm{Y}^{-} \rightarrow(\mathrm{M}+(n-2) \mathrm{H})^{(n-2)+}+\mathrm{YH}_{2}^{+}
$$

Figure 9 shows an example of positive ion data acquired when positive ion electrospray of horse heart myoglobin injected into one arm of the Y-tube was effected without the admission of negative ions (top), and when negative ion electrospray of fluorescein into the other arm of the Y-tube was effected simultaneously with positive ion electrospray of myoglobin (bottom). The dominant ion in the negative ion electrospray mass spectrum (not shown) was the deprotonated molecule $(\mathrm{M}-\mathrm{H})^{-}$at $\mathrm{m} / \mathrm{z}$ 331. Note the prominent product at $\mathrm{m} / \mathrm{z} 333$, which corresponds in mass-to-charge to protonated fluorescein, in the ion/ion positive ion mass spectrum (bottom panel of Fig. 9). This peak was essentially absent in the positive ion mass spectrum of myoglobin (top panel of Fig. 9). Similar observations were made with deprotonated ATP in reactions with highly charged protein positive ions. The charge inversion phenomenon, however, was not observed when the positively charged reactants were singly protonated positive ions nor for doubly protonated bradykinin. Based on these and a number of other observations not discussed here, a strong case was made that the most likely mechanism for the formation of protonated fluorescein and protonated ATP involved double proton transfer in a single encounter. This mechanism requires the formation of a relatively long-lived collision complex, whereby the first proton transfer presumably involves neutralization of the negative charge site followed by proton transfer from the highly charged protein to the newly formed neutral species.
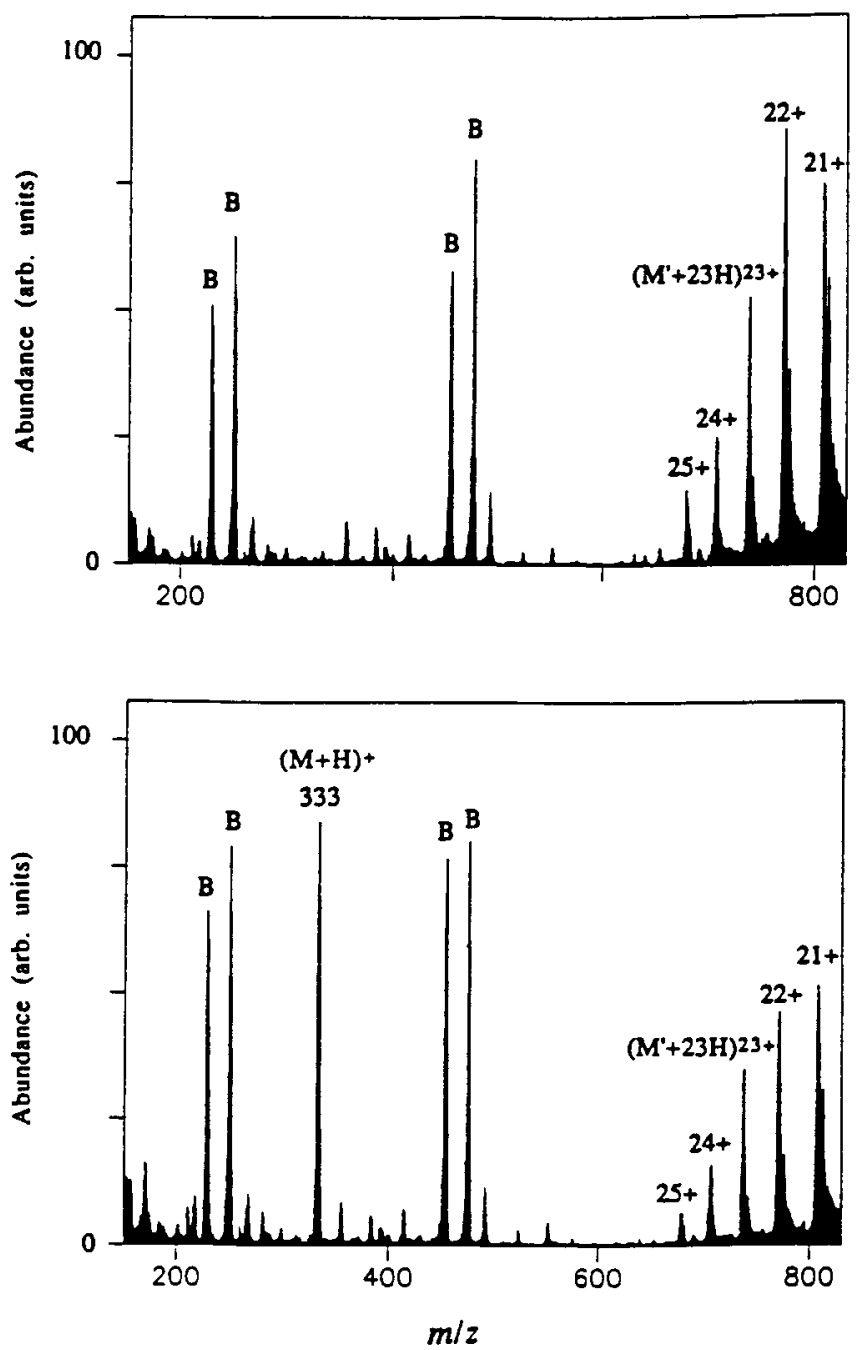

FIGURE 9. Positive ion electrospray mass spectra of horse heart myoglobin (top) before and (bottom) after negative ion electrospray of fluorescein (M) was effected in the opposing arm of the Y-tube reactor (B =background). (Reprinted with permission from J Phys Chem 1991, 95, 6412-6415, copyright 1991 American Chemical Society.)

No ion trap experiments have yet led to the observation of double proton transfer. None of the ion trap studies conducted to date has involved the use of negative ions that, once neutralized, are sufficiently basic to expect them to remove an additional proton via ion/molecule proton transfer. However, the demonstration that anion attachment can lead to the formation of a proton transfer intermediate (see Section II.A.3) shows that the formation of a relatively long-lived ion/ion intermediate is likely to play a role in the reaction dynamics of at least some types of ion/ion reactions.

\section{Solvent Transfer with Proton Transfer}

The second Y-tube study described an interesting observation, whereby the presence of negative ions in the 

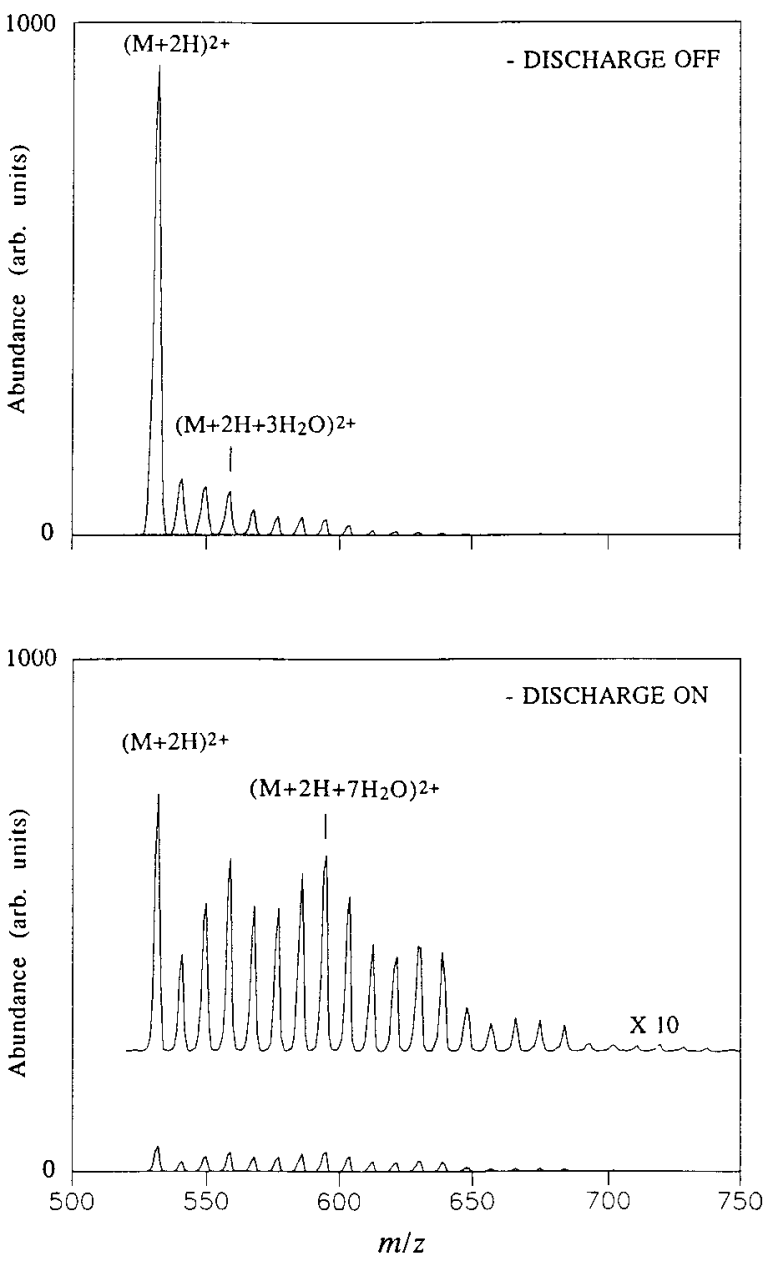

FIGURE 10. Positive ion electrospray mass spectra of bradykinin over the $m / z$ range of 500-750 without (top) and with (bottom) negative corona discharge operated in the opposing arm of the Y-tube reactor. (Reprinted from the J Am Soc Mass Spectrom 1992, 3, 695-705, with permission from Elsevier Science, Inc. Copyright 1992 by the American Society for Mass Spectrometry.)

Y-tube resulted in the partial reduction of protein positive ion charge states and in an increased relative degree of solvation of the protein ions. Figure 10 provides an example of bradykinin positive ions. Mass spectra are shown over the mass-to-charge region of the doubly charged ion with and without the operation of a negative discharge in the arm of the Y-tube not used for positive ion electrospray. There is clearly an increase in the relative abundances of doubly charged ions with 1-17 water molecules attached, when the negative ion discharge is in operation. The reduction in the $(\mathrm{M}+2 \mathrm{H})^{2+}$ signal could be attributed largely to reduction to singly charged ions. Much of the solvated doubly charged ion signal was attributed to proton transfer from, and solvent transfer to, the triply protonated bradykinin ions (not shown in Fig. 10). A single collision process that leads to this kind of observation might be written as:

$$
\begin{aligned}
(\mathrm{M}+n \mathrm{H})^{n+}+\mathrm{YS}_{m}^{-} \rightarrow(\mathrm{M}+(n-1) \mathrm{H})^{(n-1)-} \cdot( & (m-x) \mathrm{S} \\
& +\mathrm{YH}+x \mathrm{~S}
\end{aligned}
$$

where S represents a solvent molecule. This mechanism assumes that a solvated anion is one of the reactants [the major negative ion reactants that result from the discharge were $\mathrm{O}_{2}^{-}\left(\mathrm{H}_{2} \mathrm{O}\right)_{n}$ ions] and that solvent is transferred within the collision complex that leads to proton transfer. It is not clear from the Y-tube studies that a single collision mechanism is responsible for this observation. Reaction (8), therefore, remains to be demonstrated unambiguously. None of the ion trap experiments as yet can address this issue directly, because no solvated anions have thus far been used as negative ion reagents.

\section{B. Multiply Charged Negative lons/Singly Charged Positive Ions}

All the multiply charged negative ions studied thus far have been deprotonated species. The generic multiply charged negative ion is represented herein as $(\mathrm{M}-\mathrm{nH})^{\mathrm{n}-}$. Positive ion reactions have generally either been protonated molecules or radical cations, and are represented generically as $\mathrm{YH}^{+}$and $\mathrm{Y}^{+\bullet}$, respectively.

\section{Proton Transfer}

Relatively small multiply charged oligonucleotide anions and anions derived from oxidized bovine insulin Achain have been studied with the Y-tube reactor approach (Ogorzalek-Loo \& Smith, 1992) and with a quadrupole ion trap (Herron et al., 1995a; Herron et al., 1996b). In the former case, the oligonucleotide anions were reacted with a mixture of solvated protons (protonated water clusters, protonated methanol clusters, and mixed solvent clusters) produced by an atmospheric pressure discharge. In addition, anions derived from oxidized bovine insulin A-chain were reacted with multiply protonated myoglobin ions. For the latter case, protonated pyridine formed by self-chemical ionization in the ion trap served as the cationic reactant for the oligonucleotide and polypeptide anions. In both cases, the major reaction type was observed to be proton transfer, written generically as:

$$
(\mathrm{M}-n \mathrm{H})^{n-}+\mathrm{YH}^{+} \rightarrow(\mathrm{M}-(n-1) \mathrm{H})^{(n-1)-}+\mathrm{Y}
$$

There was evidence for the production of a fragment ion from anions of $5^{\prime}-\mathrm{d}(\mathrm{pAAA})-3^{\prime}$ in the Y-tube study. This product might have resulted from the exothermicity of the proton transfer reaction. However, firm conclusions on the origin of the possible fragment ion cannot be drawn from the Y-tube studies. No clear indication of fragmentation of the negative ions as a result of proton transfer reactions with 
protonated pyridine was evident in the ion trap MS/MS studies. However, different cationic reagents were used and the oligonucleotide was $5^{\prime}$-d(AAAA)- $3^{\prime}$. In the ion trap studies, all the even-electron anions studied thus far appear to react exclusively with protonated pyridine via proton transfer. The only instances of identifiable fragmentation that result from proton transfer from protonated pyridine to multiply charged anions were in the cases of doubly deprotonated 2-hydroxynaphthalene-3,6-disulfonic acid (Herron et al., 1996a), and the doubly deprotonated dye Direct Red 81 (Herron et al., 1995b). As discussed in more detail in Section IV, ion/ion reaction-induced fragmentation is most likely to be observed in the ion trap with relatively small ions; that finding is consistent with the fragmentation that was observed for the latter two anions, but not for the larger oligonucleotide and polypeptide anions.

\section{Electron Transfer}

Although electron transfer reactions that involve multiply protonated positive ions have rarely been observed thus far, electron transfer reactions that involve multiply charged closed-shell negative ions are straightforward to effect (Herron et al., 1996; McLuckey et al., 1997; Stephenson et al., 1997). This reaction can be effected by selecting positive ion reagents that cannot react readily by proton or other cation transfer. In most studies to date, rare-gas cations have been used to study electron transfer reactions. The reaction can be written generically as:

$$
(\mathrm{M}-n \mathrm{H})^{n-}+\mathrm{Y}^{+\cdot} \rightarrow(\mathrm{M}-n \mathrm{H})^{(n-1)-\cdot}+\mathrm{Y}
$$

Extensive dissociative electron transfer has been observed for relatively small polyanions by using rare gas cations. The combination of high reaction exothermicities (see Section IV) and relatively small ion size maximizes the probability for observing fragmentation. Too little is known in general about the relative kinetic stabilities of radical anions and closed shell anions of the same charge state derived from the same precursor molecule to conclude whether the inherent stabilities of electron transfer products also play a role in the observation of dissociative electron transfer ion/ion reactions.

Figure 11 shows the ion/ion MS/MS spectrum that results from the storage of mass-selected $(\mathrm{M}-2 \mathrm{H})^{2-}$ ions derived from $5^{\prime}$-d(AAA)-3' with xenon cations for $200 \mathrm{~ms}$. This spectrum illustrates the extent to which dissociative electron transfer can occur with relatively small oligonucleotide anions that react with rare gas cations. In this case, the initially formed singly charged radical anion $(\mathrm{M}-2 \mathrm{H})^{-}{ }^{\bullet}$, which results from transfer of an electron to a xenon cation, is not reflected in the spectrum (see the dashed vertical line that indicates where this product should be located on the

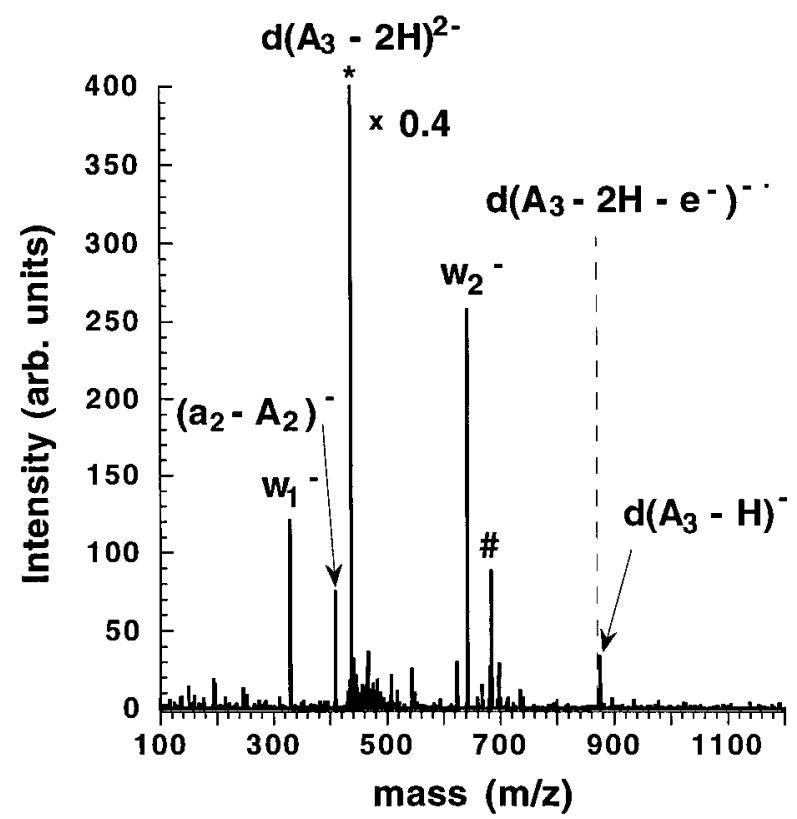

FIGURE 11. Ion/ion electron transfer spectrum arising from interaction of the $(\mathrm{M}-2 \mathrm{H})^{2-}$ ion of $5^{\prime}-\mathrm{d}(\mathrm{AAA})-3^{\prime}$ with xenon cations for $200 \mathrm{~ms}$. (Reprinted with permission from J Am Chem Soc 1995, 117, 11555-11562, copyright 1995 American Chemical Society.)

mass-to-charge scale). Some singly charged even-electron ions, $(\mathrm{M}-\mathrm{H})^{-}$, are observed, which result from a competing ion/molecule proton transfer reaction that arises from residual neutral acidic species present that are in the vacuum system. As has been observed for a number of relatively small anions, essentially all the initially formed radical anion products fragment as a result of the relatively high exothermicity of the electron transfer reaction. As the size of an ion becomes larger, however, the extent of dissociative electron transfer decreases, as reflected in Fig. 12. The spectrum of Fig. 12 shows the result of an MS/MS spectrum that involves the reaction of a population of $6-$ anions (viz., $\left(\mathrm{M}-6 \mathrm{H}^{6-}\right.$, $(\mathrm{M}-7 \mathrm{H}+\mathrm{Na})^{6-}$, and $\left.(\mathrm{M}-8 \mathrm{H}+2 \mathrm{Na})^{6-}\right)$ derived from $5^{\prime}-$ d(GTCTTAGCGCTAAGAC)-3' with xenon cations. In contrast with the smaller oligonucleotide anions, very little dissociative electron transfer is noted. As discussed further in the next section, the extent to which dissociative electron transfer occurs is determined by the kinetic stability of the initially formed ion/ion reaction product, the ion/ion reaction exothermicity, and the size of the product ion. Dissociation tends to be minimized as reaction exothermicity decreases and as product ion size increases. The data in Figs. 11 and 12 illustrate the general trend that fragmentation tends to decrease as the size of the product ion increases.

\section{Cation Attachment}

In analogy with anion attachment to multiply charged positive ions, anion attachment to multiply charged negative 


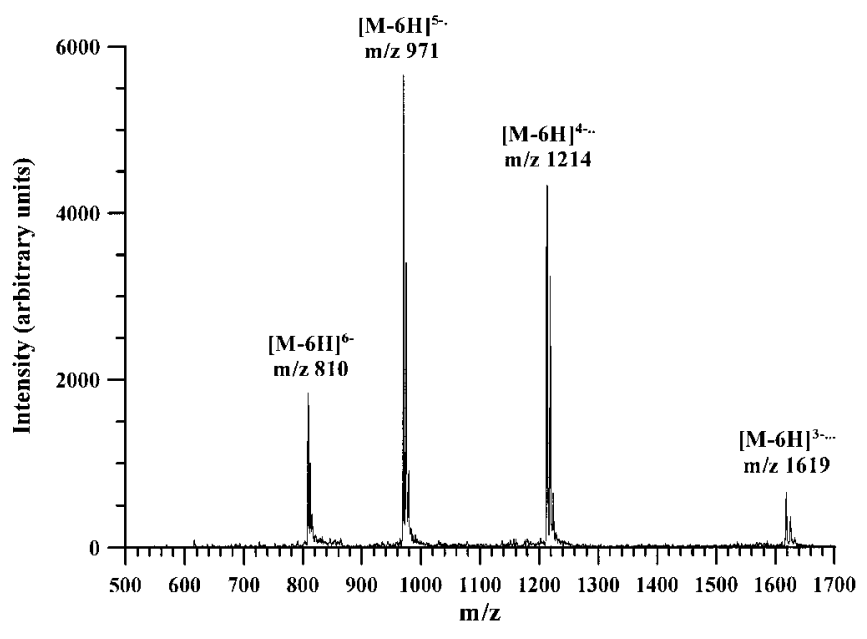

FIGURE 12. Post-ion/ion reaction spectrum (negative ion) obtained after the -6 ions derived from $5^{\prime}$-d(GTCTTAGCGCTAAGAC)-3' were reacted with xenon cations for $60 \mathrm{~ms}$. (Reprinted from Rapid Commun Mass Spectrom 1997, 11, 875-880 with permission. Copyright 1997 John Wiley \& Sons, Ltd.)

ions has been observed. The reaction can be written generically as:

$$
(\mathrm{M}-n \mathrm{H})^{n-}+\mathrm{Y}^{+} \rightarrow(\mathrm{M}-n \mathrm{H}+\mathrm{Y})^{(n-1)-}
$$

In the course of studying the proton transfer reactions that involve pyridine and deprotonated negative ions derived from oxidized bovine insulin, the first observation of cation attachment to a multiply charged negative ion was made (McLuckey et al., 1996a). Figure 13 shows the negative ion electrospray mass spectrum of oxidized bovine insulin Achain before and after reaction with a mixture of cations formed from the ion trap self-chemical ionization of pyridine. The peaks labeled in Fig. 13(b) as $\alpha$ are ion/ion attachment products comprised of the polypeptide with a cation of empirical formula $\mathrm{C}_{9} \mathrm{H}_{8} \mathrm{~N}^{+}$. The peaks labeled as $\beta$ are products comprised of the polypeptide with two cations of empirical formula $\mathrm{C}_{9} \mathrm{H}_{8} \mathrm{~N}^{+}$. It was noted that when the low $\mathrm{m} / \mathrm{z}$ cutoff of the ion trap was held above $\mathrm{m} / \mathrm{z}$ 52, the only major cation observed was protonated pyridine, and the only ion/ion reaction observed was proton transfer [reaction (9)]. However, when the low $\mathrm{m} / \mathrm{z}$ cutoff was held below $\mathrm{m} / \mathrm{z}$ 52 , an abundant cation at $\mathrm{m} / z, 130$ was observed; this cation reacted by attachment to the polypeptide anions. MS/MS studies confirmed that this ion originated from the previously described ion/molecule reaction (Lifshitz et al., 1981):

$$
\mathrm{C}_{4} \mathrm{H}_{4}^{+\cdot}+\mathrm{C}_{5} \mathrm{H}_{5} \mathrm{~N} \rightarrow \mathrm{C}_{9} \mathrm{H}_{8} \mathrm{~N}^{+}+\mathrm{H}^{\cdot}
$$

Interestingly, the isomeric $\mathrm{C}_{9} \mathrm{H}_{8} \mathrm{~N}^{+}$ion derived from protonation of quinoline reacted exclusively by proton transfer with the oligopeptide anions. Collision-induced dissociation experiments indicated that the two forms of $\mathrm{C}_{9} \mathrm{H}_{8} \mathrm{~N}^{+}$were distinct. The different ion/ion reactivities of these two forms of $\mathrm{C}_{9} \mathrm{H}_{8} \mathrm{~N}^{+}$were interpreted as being due to structural differences, such that a barrier to proton transfer was present in the energy surface involved in the attachment of the ion/ molecule reaction product that was absent in the case of protonated quinoline. Although the absolute structure of the $\mathrm{C}_{9} \mathrm{H}_{8} \mathrm{~N}^{+}$ion that reacted by attachment was not determined, the formation of an $\mathrm{N}$-alkylated pyridinium ion, such as $\mathrm{HC} \equiv \mathrm{C}-\mathrm{CH}=\mathrm{CH}-\mathrm{N}^{+} \mathrm{C}_{5} \mathrm{H}_{5}$, is consistent with the collision-induced dissociation data and with a study by $\mathrm{Yu}$ et al (Yu et al., 1993).
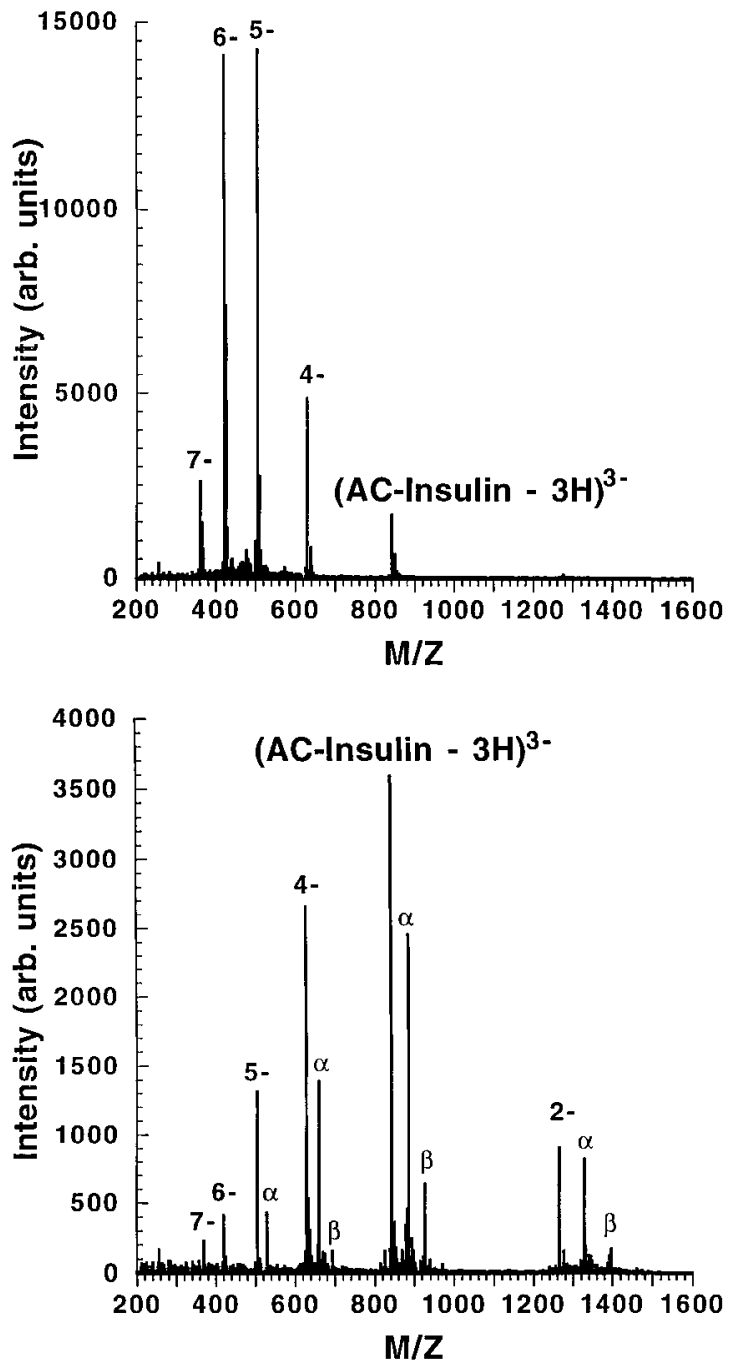

FIGURE 13. Negative ion electrospray mass spectra of oxidized bovine insulin A-chain (a) before and (b) after storage with cations derived from electron ionization of pyridine. Under the reaction conditions used to obtain (b), protonated pyridine and the $\mathrm{C}_{9} \mathrm{H}_{8} \mathrm{~N}^{+}$ion/molecule reaction product were the most abundant cations in the ion trap. (Reprinted from $\mathrm{J}$ Mass Spectrom 1996, 31, 1093-1100 with permission. Copyright 1996 John Wiley \& Sons, Ltd.) 

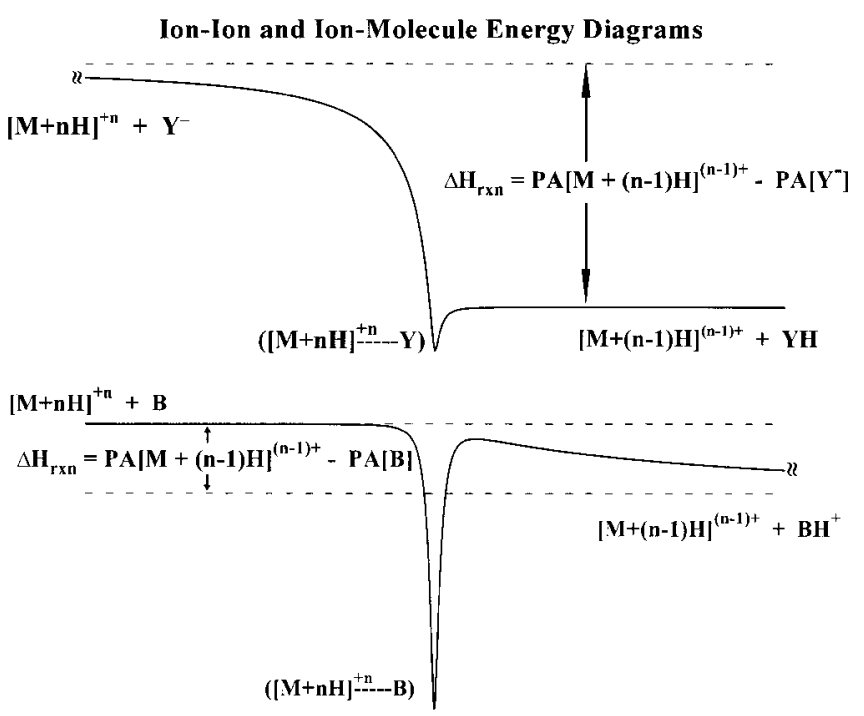

FIGURE 14. Generic energy diagrams (top) for an ion/ion proton transfer reaction and (bottom) for an ion/molecule proton transfer reaction. (Adapted with permission from J Am Chem Soc 1996, 118, 7390-7397, copyright 1996 American Chemical Society.)

\section{THERMODYNAMICS AND KINETICS}

\section{A. Thermodynamics}

To provide a context for discussing the kinetics and thermodynamics of multiply charged ion/ion reactions, it is useful to compare and contrast the major features of the energy surfaces associated with ion/ion reactions and multiply charged ion/molecule reactions. Proton transfer that involves multiply protonated positive ions is used to illustrate the general phenomenology for all "charge transfer" reactions. Figure 14 shows generic energy diagrams for the ion/ion proton transfer reaction [see Reaction (1)] and for the ion/molecule proton transfer reaction:

$$
(\mathrm{M}+n \mathrm{H})^{n+}+\mathrm{B} \rightarrow(\mathrm{M}+(n-1) \mathrm{H})^{(n-1)+}+\mathrm{BH}^{+}
$$

where B represents a strong neutral gaseous base. Both diagrams are drawn in analogy with the Brauman diagram (Brauman, 1995), whereby the zero impact parameter trajectories for the entrance and exit channels are plotted in the same plane, and the reaction coordinate proceeds from left (entrance) to right (exit). The entrance channel for the ion/ ion reaction is dominated by the long-range attractive $-\mathrm{Z}_{1} \mathrm{Z}_{2} e^{2} / \mathrm{r}$ potential, where $e$ is the electron charge, $\mathrm{Z}_{1}$ and $Z_{2}$ are the unit charges of the cations and anions, respectively, and $r$ is the interparticle separation. Assuming that one of the products is completely neutralized, the exit channel is dominated by the much shorter range ion-dipole and ion-induced dipole potentials.

The entrance channel for a multiply charged ion/mole- cule proton transfer reaction is governed at long range by relatively short-range attractive polarization forces, whereas the exit channel (in which two products of like charge are formed) is dominated by a repulsive $+\mathrm{Z}_{1} \mathrm{Z}_{2} e^{2} / \mathrm{r}$ potential at long range. This Coulombic repulsion creates a barrier in the exit channel, the magnitude of which is determined by the strength of the Coulomb field at the transition state. This situation has important implications in the ion/molecule chemistry associated with multiply charged ions, and has been discussed within the context of the chemistry of small multiply charged ions (for example, see Tonkyn \& Weisshaar, 1986; Roth \& Freiser, 1991; Petrie et al.; 1993; Baranov \& Bohme, 1997) and high-mass multiply charged ions (for example, see Williams, 1996; Cassady \& Carr, 1996; McLuckey et al., 1995b).

It is clear that the shapes of the energy surfaces of ion/ion and ion/molecule reactions that involve multiply charged ions are very different. The entrance channel of the ion/ion reaction is dominated by an attractive Coulomb potential, and the exit channel looks like that of an ion/molecule reaction. Although there may be a chemical barrier in the exit channel of an ion/ion reaction, as proposed to explain the attachment of the ion/molecule reaction product $\mathrm{C}_{9} \mathrm{H}_{8} \mathrm{~N}^{+}$to deprotonated oxidized bovine insulin discussed in Section III.B.3, there is no Coulomb barrier. The entrance channel for ion/molecule proton transfer, on the other hand, is dominated by polarization potentials, whereas the exit channel is dominated by a repulsive Coulomb potential at $r$ values greater than that of the transition state. Another major difference in the appearance of the Brauman diagrams for the two reaction types arises from the significantly different energetics associated with them. In the case of the ion/ion reaction, the free energy of the reaction is determined by the difference in the gas-phase basicity (GB) of $[\mathrm{M}+(\mathrm{n}-1) \mathrm{H}]^{(\mathrm{n}-1)+}$ and the GB of $\left[\mathrm{Y}^{-}\right]$(or its equivalent, the gas phase acidity of $\mathrm{YH})$, i.e.:

$$
\Delta \mathrm{G}_{r x n}=\mathrm{GB}\left[(\mathrm{M}+(n-1) \mathrm{H})^{(n-1)+}\right]-\mathrm{GB}\left[\mathrm{Y}^{-}\right]
$$

and the enthalpy of the reaction is determined by the difference in the relevant proton affinities (PA), i.e.:

$$
\Delta \mathrm{H}_{r x n}=\mathrm{PA}\left[(\mathrm{M}+(n-1) \mathrm{H})^{(n-1)+}\right]-\mathrm{PA}\left[\mathrm{Y}^{-}\right]
$$

Although relatively few quantitative measurements of proton affinities and/or gas-phase basicities of cations have been made (see Schnier et al., 1995; Kaltashov \& Fenselau, 1995) (such measurements using ion/molecule reactions, for example, are complicated by the Coulomb barrier in the exit channel), deprotonation of even the strongest of gaseous bases by singly charged anions is expected to be highly exothermic, because a significant gap exists in energy between the highest proton affinities of neutral bases and the 
lowest proton affinities of anions. The effect of multiple protonation is to reduce the stabilization that is associated with the addition of each successive proton. Therefore, deprotonation via reaction (1) is expected to be exothermic for every value of $\mathrm{n}$, usually by $100 \mathrm{kcal} / \mathrm{mol}$ or greater.

In the case of ion/molecule proton transfer reactions, reaction enthalpy is determined by the difference between the proton affinities of $(\mathrm{M}+(\mathrm{n}-1) \mathrm{H})^{(\mathrm{n}-1)+}$ and $\mathrm{B}$, viz.:

$$
\Delta \mathrm{H}_{r x n}=\mathrm{PA}\left[(\mathrm{M}+(n-1) \mathrm{H})^{(n-1)+}\right]-\mathrm{PA}[\mathrm{B}]
$$

Because $\mathrm{PA}\left[\mathrm{Y}^{-}\right] \gg \mathrm{PA}[\mathrm{B}]$ in general, reaction (1) is always expected to be far more exothermic than reaction (13). In fact, reaction (13) may well be endothermic even for strong neutral bases in reaction with low charge states of moderateto-large proteins. Furthermore, the energy surface associated with the ion/molecule reaction may also reduce the rate of reaction (13) by making it endoergic, even if it is slightly exothermic overall.

\section{B. Kinetics}

Considerable effort has gone into development of theories that predict rates of reaction of singly charged ions of opposite polarity over a wide pressure range. The reviews mentioned in the introduction are largely devoted to theoretical aspects of reaction kinetics of singly charged ions (Bates, 1985; Flannery, 1982a; Flannery, 1982b; Smith et al., 1976; Mahan, 1973). The first model used to predict the dependence of reaction rate on charge state for multiply charged ion/ion reactions was developed in analogy with derivations for ion/molecule orbiting collisions. That is, it is assumed that the rate constant for reaction is equal to the rate constant for forming a stable orbiting complex, $\mathrm{k}_{\mathrm{c}}$. Assuming the long range attractive potential to be the Coulomb potential, $\mathrm{k}_{\mathrm{c}}$ is:

$$
k_{c}=\nu \pi\left[\mathrm{Z}_{1} \mathrm{Z}_{2} e^{2} /\left(\mu \nu^{2}\right)\right]^{2}
$$

where $\nu$ is the relative velocity of the oppositely charged ions, $\mu$ is the reduced mass of the collision partners, and $\mathrm{Z}_{1}$ and $Z_{2}$ are the charges of the cations and anions, respectively. This picture makes the assumption that the high exothermicity of the reaction ensures that the oppositely charged ions will react once they form a complex. This picture is not totally satisfying, because, as a result of the long range $1 / \mathrm{r}$ attraction, a multiply charged ion and an oppositely charged ion can form a stable orbiting complex at values of $r$ that is significantly larger than typical distances over which a proton or electron can jump. Furthermore, "recombination" of the ions as with anion or cation attachment often requires much closer interaction than the calculated capture radius. Once the ions are in orbit, some mech- anism for reducing $r$ to the point where chemical reaction can occur is required, and this factor is not made explicit in Eq. (17). Collisions with the background helium present at roughly 1 mtorr in the ion trap are believed to serve an important role in facilitating ion/ion reactions. A collision of one of the ions that comprises an orbiting complex with a third body can either destroy the collision complex, or it can serve to reduce the relative velocity of the collision partners; thus, the orbiting radius is reduced. The low mass of helium results in minimal scattering of significantly higher mass collision partners, so that the net effect of using a low mass bath gas is to reduce ion velocities upon collision without leading to significant changes in their trajectories. For this reason, helium is expected to be a particularly efficient third body for promoting ion/ion chemistry.

Use of Eq. (17) to model ion/ion reaction rate constants implicitly assumes that all ions that undergo ion/ion capture eventually react. This assumption requires that the bath gas be $100 \%$ efficient at bringing ions into sufficiently close proximity for reaction. Although making rate measurements as functions of bath gas pressure and identity might shed light on the validity of this assumption, such measurements have not been made and are difficult to make, due to the important roles that the bath gas plays in other parts of the ion trap experiment. Furthermore, the measurement of absolute rate constants is hampered by uncertainty in the ion densities associated with the experiment; thus, quantitative comparisons between measurements and predictions of Eq. (17) are difficult to make. However, it is straightforward to admit a large excess of singly charged ions so that pseudofirst order kinetics are observed. Under such conditions, measurement of reaction rates is straightforward. Figure 15 shows a plot of ion/ion proton transfer rate as a function of the square of the charge of multiply charged ubiquitin ions. These data were collected by isolating a specific charge state of multiply protonated ubiquitin, subjecting the ions to reaction with a large excess of PDCH anions (to ensure pseudo-first order kinetics), and deriving the rate from the slope of the plot of the negative logarithm of the $\mathrm{I}_{\mathrm{t}} / \mathrm{I}_{0}$ intensity ratio vs. time. These data confirm the prediction of Eq. (17) that ion/ion reaction rates follow a charge-squared dependence. It is also worth noting that, in those cases in which assessments of rates vs. charge could be made, a similar dependence of reaction rate upon multiply charged ion charge has been observed for all reaction types observed in ion trap experiments. Such an observation is expected for reactions where the formation of an orbiting complex is rate-limiting.

It is instructive to compare and contrast the kinetics for proton transfer from multiply protonated ions to negative ions (viz., ion/ion reactions), vs. proton transfer from multiply protonated ions to strong neutral bases (viz., ion/molecule reactions). Figure 16 shows a plot of proton transfer rate to anions derived from PDCH (filled squares) and to $\mathrm{N}, \mathrm{N}, \mathrm{N}^{\prime}, \mathrm{N}^{\prime}$-tetramethyl-1,4,-diaminobutane (filled circles) 


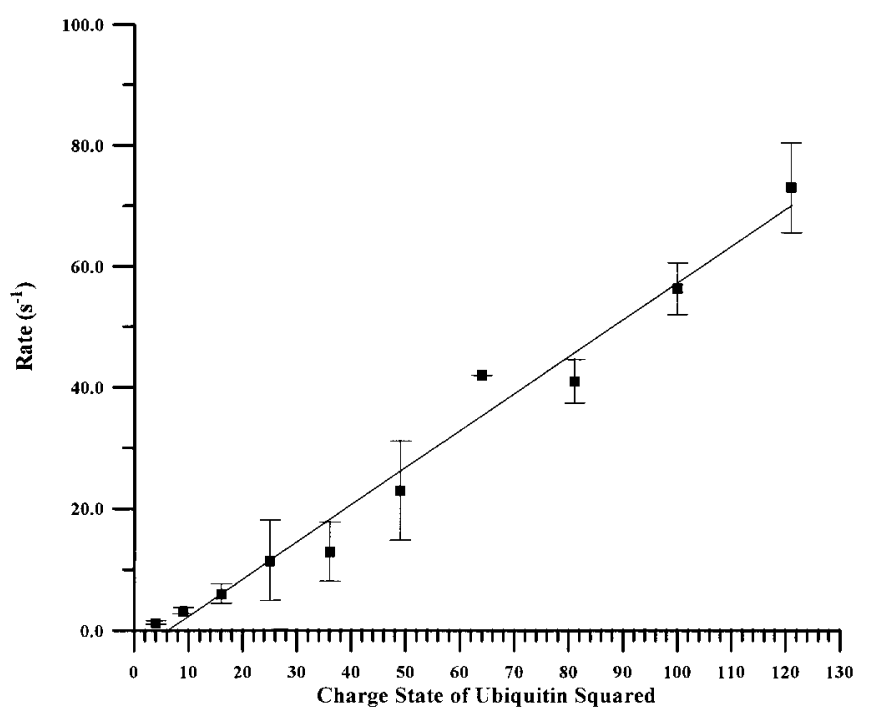

FIGURE 15. Plot of ion/ion proton transfer rate vs. the square of the charge of the bovine ubiquitin cations. (Reprinted with permission from J Am Chem Soc 1996, 118, 7390-7397, copyright 1996 American Chemical Society.)

from multiply charged ubiquitin ions as a function of ubiquitin charge. The ion/molecule reaction data were taken from the research of Cassady et al. (Cassady et al., 1994). The ion/molecule reaction results were originally reported as rate constants, but were converted here to rates by multiplying the rate constants by $6.48 \times 10^{9} \mathrm{~cm}^{-3}$, the highest number density of the neutral base reported to be used in the ion cyclotron resonance mass spectrometry studies. This conversion was done to allow for a direct comparison of the ion/ion and ion/molecule reaction results. Clearly, the rate vs. charge state behavior of the ion/molecule and ion/ion reactions are qualitatively very different. For example, the greater-than-linear decrease in rate as charge state decreases for the ion/molecule reactions reflects a decrease in reaction efficiency, which is defined as the fraction of ion/molecule collisions that lead to reaction. If it is assumed that each charge site of the ubiquitin ions reacts with equal efficiency, then the ion/molecule reaction rates should be linear with charge (Ikonomou \& Kebarle, 1992). However, the energy surface of the ion/molecule reaction is affected by the Coulomb field strength via its influence on the gas-phase basicities of the ions and on the magnitude of the Coulomb barrier (see Fig. 14). For this reason, it is possible that ion/molecule proton transfer can be exoergic at high charge states and endoergic at low charge states.

Ion/ion reaction rates also decrease with decreasing charge state; but, the shape of the curve is very different and reflects the dependence of the rate on $Z^{2}$ (see Fig. 15). The linear dependence of ion/ion proton transfer rate on $\mathrm{Z}^{2}$, as predicted by Eq. (17), suggests that the ion/ion reaction efficiency is constant for all charge states, in contrast with the ion/molecule reaction results. Such a result is to be expected given the highly exoergic nature of ion/ion reactions, and suggests that ion/ion proton transfer is a more reliable means to reduce charge states to low values than is ion/molecule proton transfer. It is noteworthy that each ubiquitin charge state appeared to react with a single rate constant in the ion/ion reaction studies. Cassady et al. have observed that ubiquitin ions of several charge states display reaction kinetics that are a convolution of two or more reacting populations (Cassady et al., 1994; Cassady \& Carr, 1996), depending upon the strength of the base. These observations have been interpreted as arising from isomeric forms of the protein. For a given charge state, different conformations can give rise to different Coulomb fields, which result in different energy surfaces for reaction. Based on the preceding arguments regarding the thermodynamics and energy surfaces associated with ion/ion vs. ion/molecule proton transfer reactions, ion/ion reaction rates are expected to be insensitive to ion structural differences.

The $\mathrm{Z}^{2}$ dependence of ion/ion reaction rates has important implications for the use of ion/ion reactions in the analysis of mixtures subjected to electrospray (McLuckey et al., 1998). First, it implies that multiply charged ions of similar mass-to-charge ratio but very different mass and charge will react at much different rates when subjected to the same population of oppositely charged ions. Therefore, overlapping charge state distributions that arise from mixtures of ions of dissimilar charge can be separated from one another by using short reaction times. Figure 17 illustrates this effect with electrospray data of a mixture of bradykinin and horse skeletal muscle apomyoglobin. Figure 17(a) shows the normal electrospray mass spectrum, wherein doubly pro-

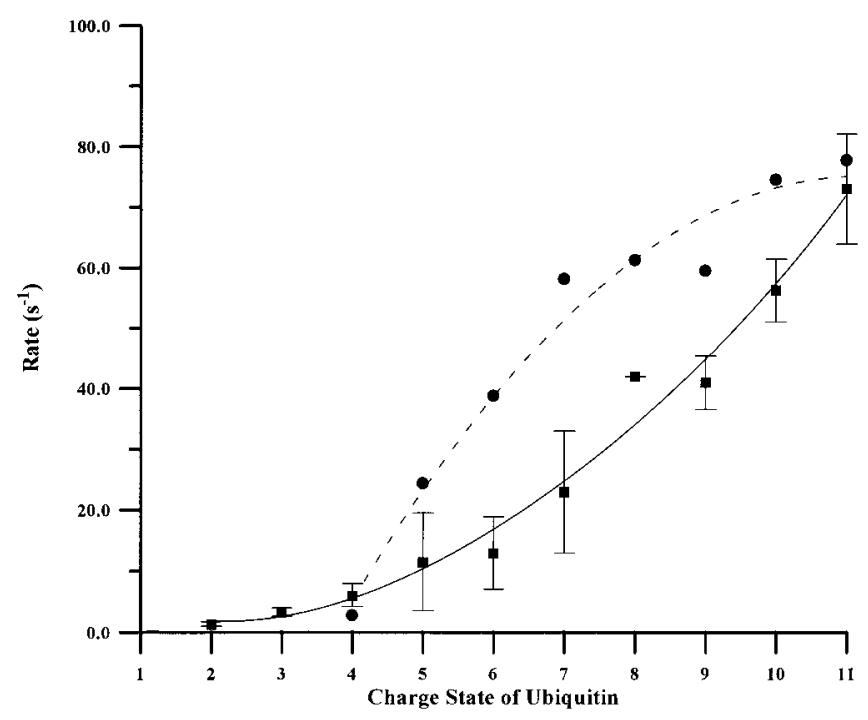

FIGURE 16. Plot of measured proton transfer rates vs. bovine ubiquitin charge state. Filled circles are ion/molecule reaction data taken from (Cassady et al., 1994) and filled squares are ion/ion reaction data. (Reprinted with permission from J Am Chem Soc 1996, 118, 7390-7397, copyright 1996 American Chemical Society.) 

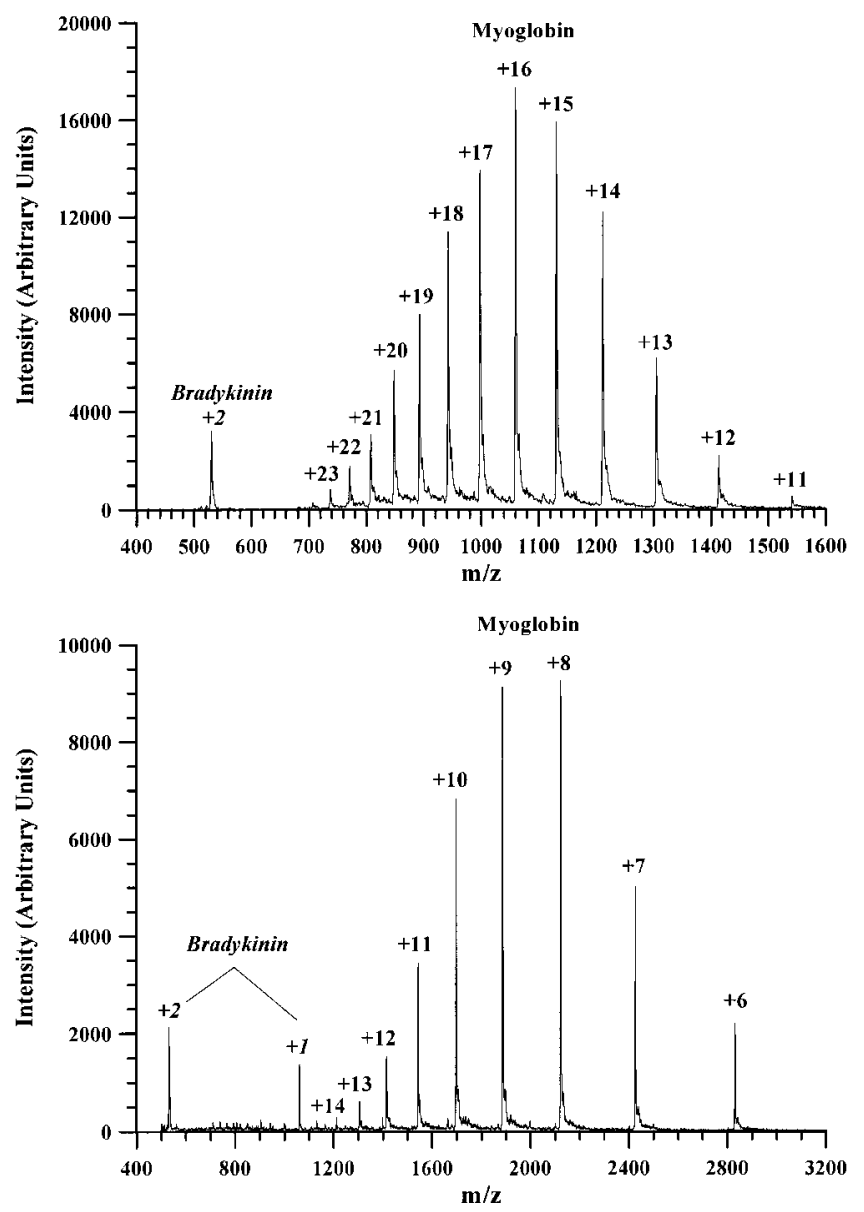

FIGURE 17. (a) Positive ion electrospray mass spectrum of a mixture of bradykinin (charge states italicized) and horse skeletal muscle apomyoglobin. (b) Positive ion electrospray mass spectrum obtained after subjecting the ions of (a) to reaction with PDCH anions for $30 \mathrm{~ms}$. (Reprinted with permission from Anal Chem 1998, 70, 1198-1202, copyright 1998 American Chemical Society.)

tonated bradykinin appears at $\mathrm{m} / \mathrm{z}$ 531, and myoglobin charge states that range from +11 to +23 appear over the $\mathrm{m} / \mathrm{z}$ range of $700-1600$. Singly protonated bradykinin, if present, is buried under the signal associated with the +16 charge state of myoglobin. Figure 17(b) shows the spectrum acquired after the mixture of ions of Figure 17(a) were subjected for $30 \mathrm{~ms}$ to ion/ion proton transfer reactions with the high mass ions derived from PDCH. In this case, doublyand singly-protonated bradykinin are both clearly distinct from the myoglobin charge-state distribution. In comparing the intensities of the signals associated with doubly protonated bradykinin prior to and after reaction, it is clear that less than half of the initial doubly charged bradykinin population was depleted by ion/ion reactions. In contrast, essentially all the myoglobin ions of charge +15 and higher are completely depleted over the same reaction period. For this depletion to occur, multiple sequential proton transfer reactions involving the highest charge states of myoglobin must go to comple- tion. Obviously, the rates of reaction of the high charge states of myoglobin are far greater than that of doubly protonated bradykinin. For this reason, the myoglobin charge-state distribution can be shifted to lower charge states very quickly, whereas the charge state distribution of bradykinin changes much more slowly.

A second, and perhaps more important, consequence of the $\mathrm{Z}^{2}$ dependence of the ion/ion reaction rates is that ions of very different initial charge states are completely neutralized over very similar time frames. Therefore, ions of a wide range of charges can be converted to low charge states by using a single ion/ion reaction period. This important consequence is illustrated in Figs. 18 and 19. Given the highly exothermic nature of mutual neutralization, ion/ion reactions can be regarded as irreversible. Therefore, it is straightforward to simulate the time evolution of the product cations via differential equations that describe a set of consecutive irreversible pseudo-first-order reactions. Under conditions in
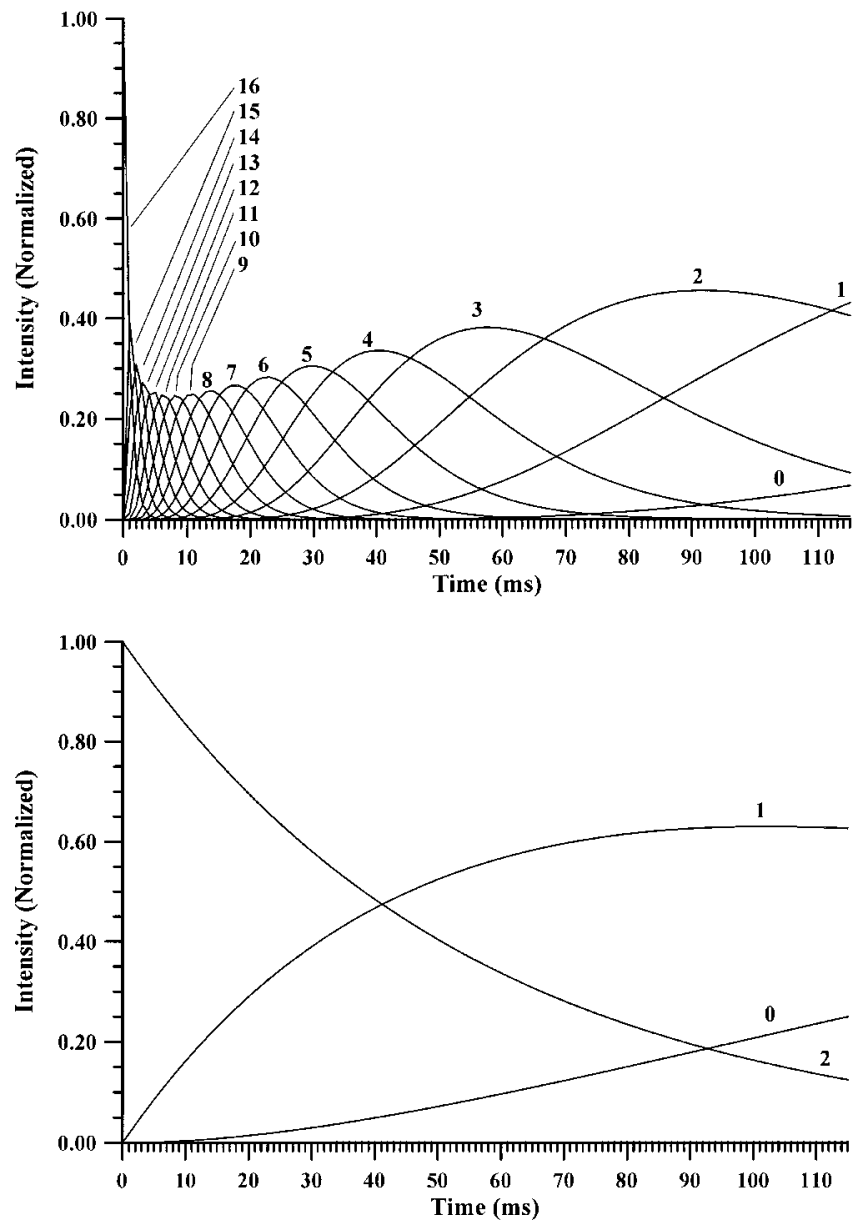

FIGURE 18. (a) Simulated ion abundance vs. reaction time curves for the $(\mathrm{M}+16 \mathrm{H})^{16+}$ ion of apomyoglobin and its products. (b) Simulated ion abundance vs. reaction time curves for the bradykinin $(\mathrm{M}+2 \mathrm{H})^{2+}$ ion and its products $(\mathrm{M}+\mathrm{H})^{+}$and $\mathrm{M}$. (Reprinted with permission from Anal Chem 1998, 70, 1198-1202, copyright 1998 American Chemical Society.) 

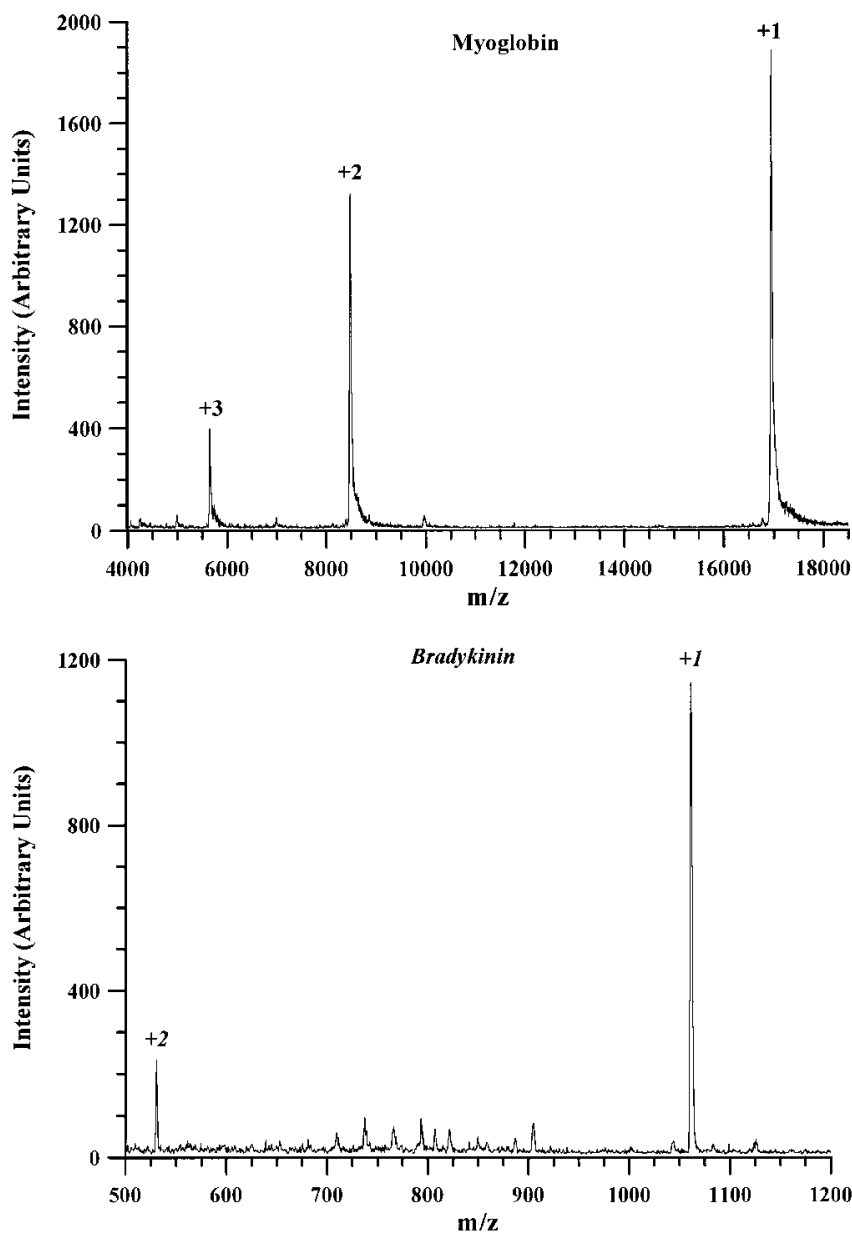

FIGURE 19. (a) Post-ion/ion spectrum $(\mathrm{m} / \mathrm{z}$ range $=4,000-18,500)$ obtained after the ions of Fig, 17(a) were subjected to reaction with PDCH anions for $115 \mathrm{~ms}$. (b) Same as (a), except the plotted $\mathrm{m} / \mathrm{z}$ range is 500-1200. (Reprinted with permission from Anal Chem 1998, 70, $1198-$ 1202, copyright 1998 American Chemical Society.)

which pseudo-first-order kinetics prevail, the time evolution of the positive ion reactants and products can be fit with a single rate, $\mathrm{R}$, that corresponds to that of the singly charged positive ion that reacts with the singly charged negative ion. The reaction rates for the higher cation charge states can simply be taken as $n^{2} R$, where $n$ is the cation charge. Figure 18 compares simulated relative abundance curves, including those of the totally neutralized species, for the +16 apomyoglobin ion and its products [Fig. 18(a)] and doubly-protonated bradykinin [Fig. 18(b)] and its products as a function of reaction time. In each case, consecutive irreversible reactions were assumed with the reaction rates for each cation taken as $n^{2} R$, where $R$ is $4.5 \mathrm{~s}^{-1}$. The very rapid growth and depletion of the higher intermediate charge states of apomyoglobin are obvious in Fig. 18(a). As the charge states decrease, the changes in the abundances of the products take place much more slowly. Under the conditions of this simulation, roughly $7 \%$ of the +16 myoglobin ions are totally neutralized after $115 \mathrm{~ms}$ of reaction. Despite the fact that bradykinin starts with an initial charge state of +2 , only about $24 \%$ of the initial ion population is totally neutralized after $115 \mathrm{~ms}$ of reaction time. As a result of the $\mathrm{Z}^{2}$ reaction rate dependence, the more highly charged apomyoglobin ions can nearly catch up to the bradykinin ions, in terms of charge state distribution, prior to total neutralization. Figure 19 shows experimental data collected from the bradykinin/ apomyoglobin mixture, using an ion/ion reaction time of 115 ms. The experimental data confirm that the +1 species from each mixture component can be the most abundant product ion in the spectrum using a single reaction time, despite the fact that one of the components was initially present largely in the +2 charge state, whereas the other component was initially present with charges that range from $+11-+23$.

\section{Ion/Ion Reactions and Fragmentation}

The preceding sections set the stage for addressing the observation, or lack thereof, of fragmentation of multiply charged ions as a result of ion/ion reactions. All ion/ion reactions are highly exothermic relative to most ion/molecule reactions. In the case of the charge transfer reactions (e.g., cation transfer, anion transfer, and electron transfer), the reaction exothermicity is partitioned between the relative translation of the products and the internal modes of the products. In the case of the attachment reactions, all the reaction exothermicity must initially reside in the ion attachment product. In this section, fragmentation associated with proton transfer and electron transfer reactions is addressed, followed by a brief discussion of unique considerations for attachment reactions.

In general, the factors that determine the extent to which fragmentation of product ions initially formed as a result of an ion/ion reaction in the quadrupole ion trap environment are: (i) reaction exothermicity; (ii) the partitioning of reaction exothermicity among all available degrees of freedom; (iii) the kinetic stability of the initially formed product ion, as determined by the enthalpic and entropic requirements for fragmentation; (iv) the number of degrees of freedom of the initially formed product ion; and (v) the rate at which excess product-ion internal energy is removed by ion/bath gas collisions and emission. The collisional cooling rate dominates under typical ion-trap conditions. Collectively, these factors determine the lifetime of the initially formed product ion and the time available for fragmentation before sufficient internal energy is removed by the cooling mechanisms to avert dissociation. The following examples and discussion illustrate the range of phenomenologies observed, and how they relate to the factors that underlie the ion/ion reaction-induced dissociation in the ion trap.

Figure 20 compares positive ion mass spectra of bovine cytochrome $c$ prior to and after reaction with the high-mass anions derived from PDCH. This comparison is in many 

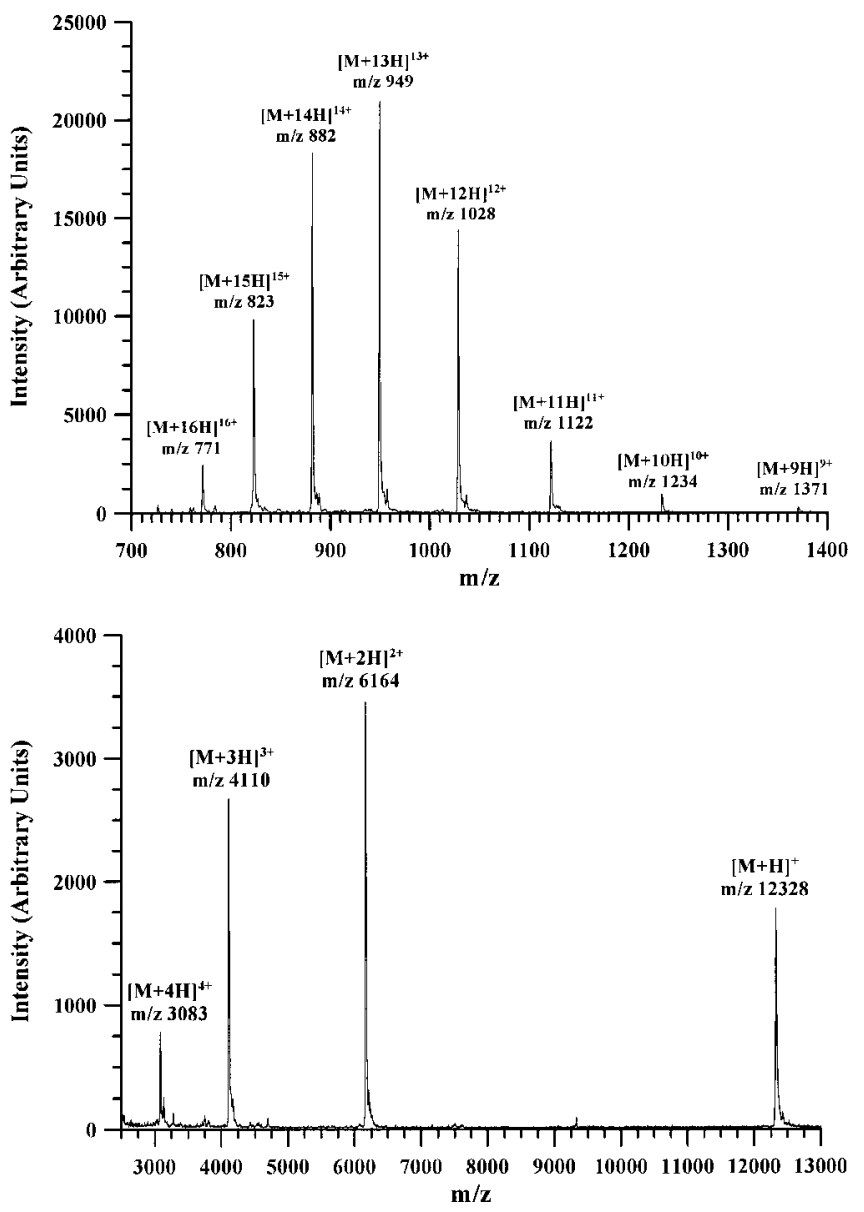

FIGURE 20. Positive ion electrospray mass spectra of bovine cytochrome c (a) before and (b) after reaction with anions derived from $\mathrm{PDCH}$ for a period of sufficient duration to produce +4 to +1 products.

ways typical for all multiply protonated polypeptides that react with perfluorocarbon anions. All charge states can be readily reduced to the +1 charge state and can be totally neutralized by use of long reaction times. Furthermore, there is no evidence for fragmentation in the post-ion/ion reaction data, despite the high exothermicity of the reactions. Figure 21 shows a plot of $\Delta \mathrm{G}_{r x n}$ as a function of cytochrome $c$ charge state, using the $\Delta \mathrm{G}_{\text {acid }}$ value for $\mathrm{C}_{6} \mathrm{~F}_{5} \mathrm{H}$ as an estimate of the gas-phase basicities of the PDCH anions and the gas-phase basicity values for multiply charged cytochrome $c$ ions as reported by Schnier et al. (Schnier et al., 1995). This plot shows that reaction exothermicity increases with the charge state of the multiply charged ion, as expected, and that even for the +3 ion, which is the lowest charge state for which a value was reported, the $\Delta \mathrm{G}_{r x n}$ is expected to be ca. $130 \mathrm{kcal} / \mathrm{mol}$. The singly charged cytochrome $c$ ions reflected in Fig. 20 were, therefore, formed from a series of consecutive proton transfer reactions, each of which ranged in $\Delta \mathrm{G}_{r x n}$ from ca. $200 \mathrm{kcal} / \mathrm{mol}$ to over $100 \mathrm{kcal} / \mathrm{mol}$. Nevertheless, there is no evidence for fragmentation of the protein.
Although there has been very little evidence observed, thus far, for fragmentation of covalent bonds of a protein ion as a result of ion/ion reactions with anions derived from $\mathrm{PDCH}$, it is perhaps even more remarkable that noncovalently bound positive ion complexes such as the myoglobin-heme complex (Stephenson et al., 1997b) and clusters of oligonucleotide single strands (Stephenson \& McLuckey, 1997c) also survive ion/ion proton transfer reactions. For example, Fig. 22 shows the results of an ion/ion proton transfer experiment that involved the +9 charge state of horse skeletal muscle holomyoglobin and the anions derived from PDCH (reaction time $=110 \mathrm{~ms}$ ) (Stephenson et al., 1997b). Loss of the noncovalently bound heme group from holomyoglobin is known to be a more facile process in gas-phase experiments than fragmentation of the covalent bonds of either the protein or the heme group (Ramsey \& McLuckey, 1994; Gross et al.,1997; Chen et al., 1998). Nevertheless, no evidence for loss of the heme group during the period associated with eight consecutive ion/ion proton transfer reactions that leads to the singly protonated complex is evident. (Loss of heme would be expected to give rise to a product ion at $\mathrm{m} / \mathrm{z} 16,951$.)

At the opposite extreme in behavior is the ion/ion electron transfer results of Fig. 11, as discussed in Section III.B.2, whereby essentially all the initially formed oddelectron oligonucleotide product anion fragmented. In the case of electron transfer [see reaction (10)], reaction enthalpies are determined by the difference between the electron affinity (EA) of $(\mathrm{M}-\mathrm{nH})^{(n-1)-\bullet}$ and the recombination energy of $\mathrm{Y}^{+\bullet}$, viz.:

$$
\Delta \mathrm{H}_{r x n}=\mathrm{EA}\left[(\mathrm{M}-n \mathrm{H})^{(n-1)-\cdot}\right]-\mathrm{RE}\left[\mathrm{Y}^{+\bullet}\right]
$$

For ground state reactants and products, the $\mathrm{RE}$ of $\mathrm{Y}^{+\bullet}$ is

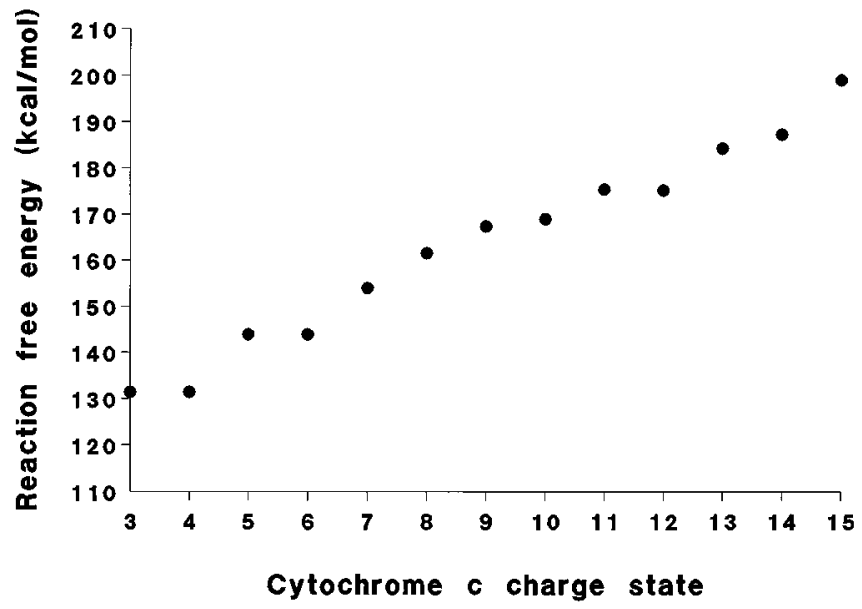

FIGURE 21. Free energy of reaction $\left(\Delta \mathrm{G}_{r x n}\right)$ as a function of cytochrome c charge state for proton transfer to $\mathrm{C}_{6} \mathrm{~F}_{5-}$. Cytochrome $\mathrm{c}$ gas-phase basicities taken from (Schnier et al., 1995). 

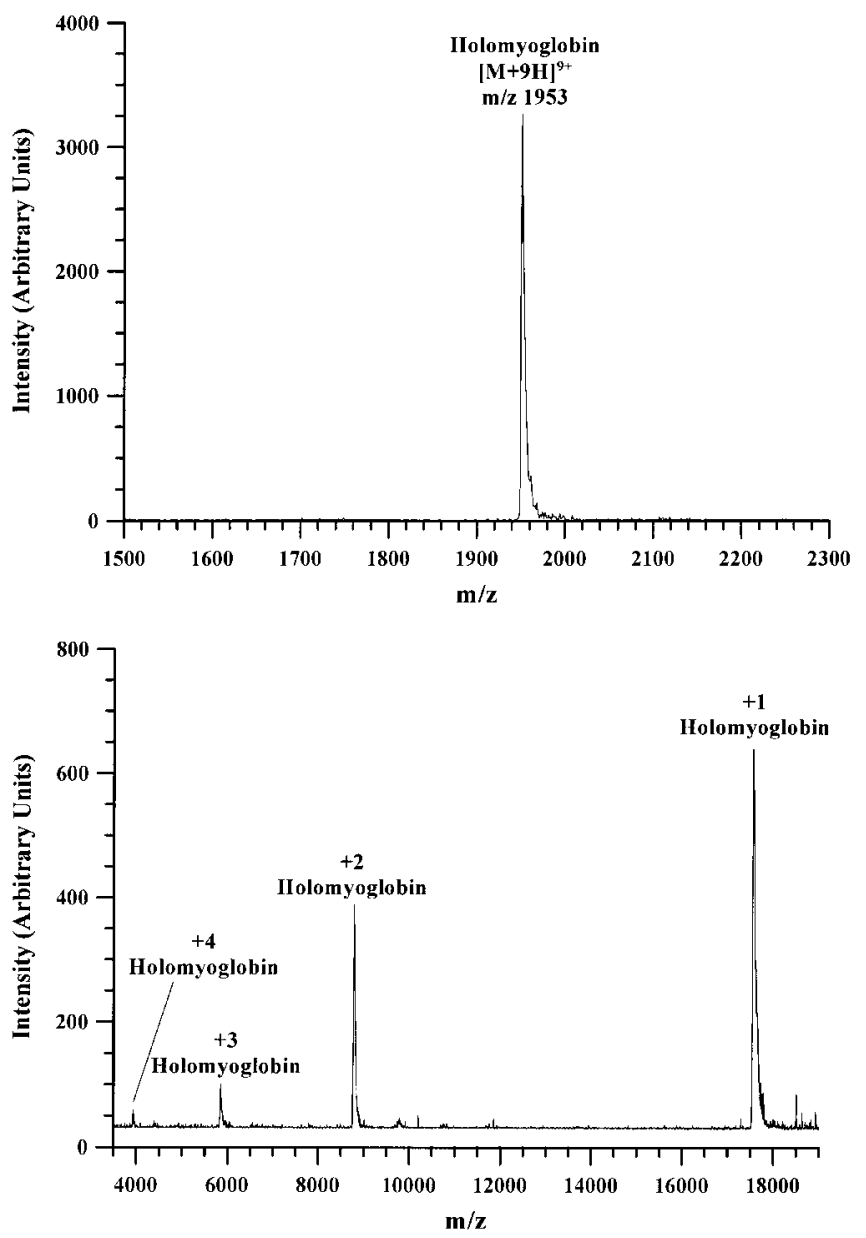

FIGURE 22. (a) Isolation of the $(\mathrm{M}+9 \mathrm{H})^{9+}$ ion of horse skeletal muscle holomyoglobin. (b) Post-ion/ion reaction positive ion mass spectrum after the $(\mathrm{M}+9 \mathrm{H})^{9+}$ ion was subjected to reaction with PDCH anions for $110 \mathrm{~ms}$. (Reprinted from the J Am Soc Mass Spectrom 1997, 8, 637-644, with permission from Elsevier Science, Inc. Copyright 1997 by the American Society for Mass Spectrometry.)

equivalent to the adiabatic ionization energy (IE) of $\mathrm{Y}$. Although electron affinities of radical anions have not been measured, they are not expected to exceed those of neutral molecules with high electron affinities (e.g., $2 \mathrm{eV}$ ) due to the destabilizing effect of the Coulomb field (Scheller at al., 1995). The IE of xenon is $12.1 \mathrm{eV}$. Therefore, the reaction that leads to Fig. 11 is expected to be exothermic by at least $10 \mathrm{eV}(230 \mathrm{kcal} / \mathrm{mol})$. This value is a higher reaction exothermicity than most proton transfer reactions due to the relatively high IE of xenon. Figure 23 compares post-ion/ion reaction data for the reaction of the $(\mathrm{M}-3 \mathrm{H})^{3-}$ anion derived from $5^{\prime}$-d(AAAA)- $3^{\prime}$ with $\mathrm{Xe}^{+\bullet}$ [Fig. 23(a)] and $\mathrm{CCI}_{3}^{+}$ [Fig. 23(b)]. In both cases, fragmentation of the initially formed $(\mathrm{M}-3 \mathrm{H})^{2-}$ production is observed, but it is far more extensive with $\mathrm{Xe}^{+\bullet}$ than with $\mathrm{CCI}_{3}^{+}$. Note that almost no $(\mathrm{M}-3 \mathrm{H})^{2-\cdot}$ signal is observed in Fig. 23(a), whereas the most abundant ion/ion reaction product in Fig. 23(b) is the formed $(\mathrm{M}-3 \mathrm{H})^{2-\bullet}$ ion. The major difference between the two experiments is that the reaction exothermicity of the $(\mathrm{M}-3 \mathrm{H})^{3-} / \mathrm{CCI}_{3}^{+}$experiment is expected to be significantly lower than that of the $\left.(\mathrm{M}-3 \mathrm{H})^{3-}\right|^{\mathrm{Xe}+\bullet}$ experiment (IE(Xe) $=12.1 \mathrm{eV} ; \mathrm{IE}\left(\mathrm{CCI}_{3}^{\circ}=7.8 \mathrm{eV}\right)$. The estimated $4.3 \mathrm{eV}$ lower exothermicity of the reaction when $\mathrm{CCI}_{3}^{+}$is used as the cationic reagent vs. when $\mathrm{Xe}^{+\bullet}$ is used highlights the role of reaction exothermicity in ion/ion reaction-induced dissociation.

The comparison of Figs. 11 and 12, which show data acquired from the reaction of a 3-mer oligonucleotide anion with ionized xenon (Fig. 11) and the reaction of a series of multiply charged 16-mer oligonucleotides (Fig. 12) highlights the role of ion size in the likelihood for fragmentation. The reaction exothermicities for these reactions are not likely to differ significantly, because most of the reaction exothermicity is derived from the recombination energy of
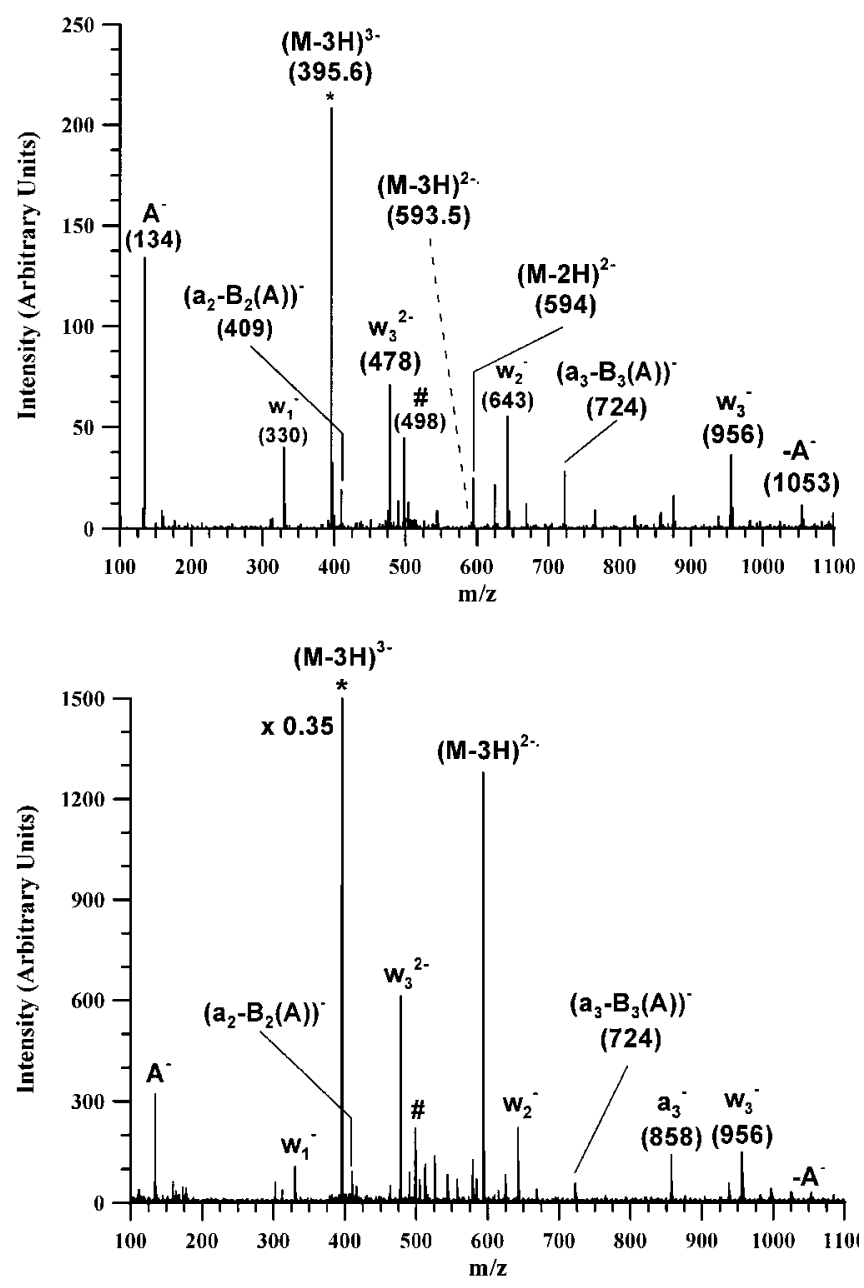

FIGURE 23. Electron transfer MS/MS spectra that results from the reaction of the $(\mathrm{M}-3 \mathrm{H})^{3-}$ anion of 5-d(AAAA)-3' with $\mathrm{Xe}^{+\bullet}$ (a) and with $\mathrm{CCl}_{3}^{+}$(b). The mass-selected precursor anion is indicated by an asterisk. A product ion that arises from loss of the $3^{\prime}$ adenine and cleavage across the $3^{\prime}$ sugar is indicated with a $£$ sign. (Reprinted from the J Am Soc Mass Spectrom 1997, 8, 148-154, with permission from Elsevier Science, Inc. Copyright 1997 by the American Society for Mass Spectrometry.) 
the cation. Rather, it is more likely that the lifetimes of the initially formed $16-$ mer ion/ion reaction products were sufficiently long for ion/helium collisions to avert dissociation. Similar arguments, as developed more fully below, can also be made with regard to the cytochrome $c$ and holomyoglobin experiments described above, and for any ion/ion reaction that involves relatively large molecules.

A key factor in minimizing fragmentation as a result of an ion/ion reaction is the possibility for collisional cooling of the initially formed excited product ions. This factor is believed to be largely responsible for the lack of a cumulative effect of multiple consecutive ion/ion reactions on energy deposition into reaction products. It is also believed to be important in inhibiting the fragmentation of product ions from a single ion/ion reaction that might otherwise dissociate within the time-frame of the ion trap experiment in the absence of a bath gas. With respect to the issue of energy deposition from multiple consecutive proton transfer reactions, the holomyoglobin experiment that led to the data of Fig. 22 can serve as an illustrative case. Using the assumption of consecutive irreversible reactions, with each charge state reacting at a rate of $n^{2} R$, where $R$ is the rate of the singly charged positive ion that reacts with the negative ions, the best fit to the time evolution of the various charge states of holomyoglobin, beginning with $(\mathrm{M}+9 \mathrm{H})^{9+}$ and leading to the spectrum of Fig. 22(b), was obtained with $\mathrm{R}=5.26 \mathrm{~s}^{-1}$. This rate, and the assumptions that the anion number density is constant and that rates scale with $\mathrm{n}^{2}$, can be used to determine the approximate average time between successive proton transfer reactions. For example, at a rate of $5.26 \mathrm{~s}^{-2}$, singly charged holomyoglobin ions, on average, undergo a collision with an anion every $190 \mathrm{~ms}$. At the other extreme, the $(\mathrm{M}+9 \mathrm{H})^{9+}$ ion reacts at a rate of $426 \mathrm{~s}^{-1}$ to yield an average time between ion/ion collisions of $2.3 \mathrm{~ms}$. Figure 24 shows a plot of the average time between ion/ion collisions as a function of cation charge state. Assuming an average collision cross-section for a holomyoglobin ion of ca. 1600 $\AA^{2}$ (Covey \& Douglas, 1993), the ion/helium bath gas collision rate is ca. $7 \times 10^{5} \mathrm{~s}^{-1}$. There are, therefore, several thousand thermalizing ion/helium collisions between proton transfer reactions for the most highly charged ions associated with the experiment that led to Fig. 22. Increasingly longer average storage times between ion/ion collisions are in effect as the cation charge state decreases such that there are, on average, 36,000 ion/helium collisions after the formation of the doubly charged ion and prior to the subsequent ion/ion collision.

Ion/ion proton transfer exothermicities are expected to be highest for the most highly charged ions (see, for example, Fig. 21) and the most highly charged ions are expected to undergo the highest rates of ion/ion collisions (see Fig. 24). It, therefore, follows that, if no fragmentation results in the first few ion/ion collisions, then it is unlikely that fragmentation will occur from the lower charge state ions, unless

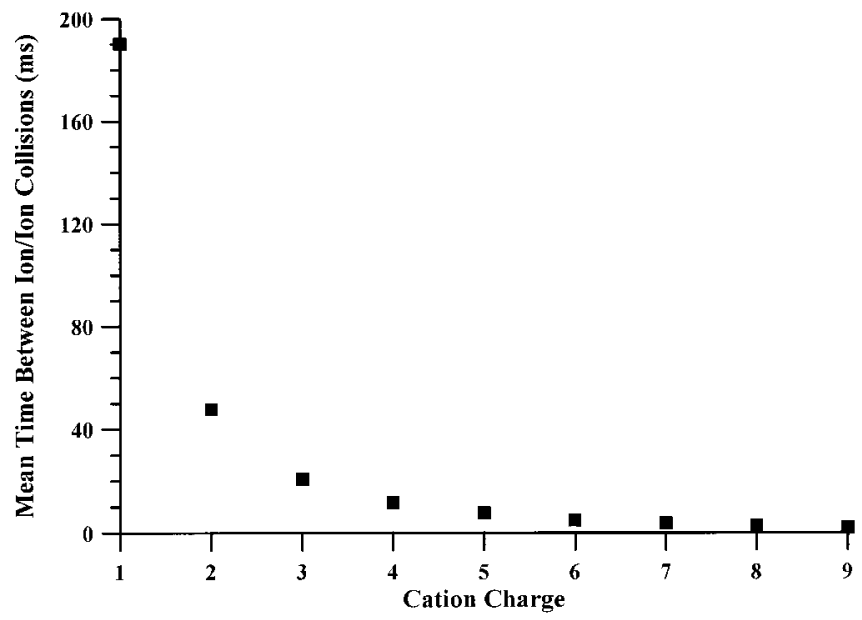

FIGURE 24. Mean time between ion/ion collisions as a function of positive ion charge state for reactions of $(\mathrm{M}+9 \mathrm{H})^{9+}$ holomyoglobin and its products with anions of PDCH. (Reprinted from the J Am Soc Mass Spectrom 1997, 8, 637-644, with permission from Elsevier Science, Inc. Copyright 1997 by the American Society for Mass Spectrometry.)

some structural change associated with the loss of charge reduces the lowest critical energy for fragmentation. In the case of holomyoglobin, the binding of the heme group in the gas phase is sufficiently strong for each charge state to survive storage in the presence of room-temperature helium for at least tens of ms, and possibly much longer.

Recent simulations of collisional relaxation of internally excited high-mass $(<1 \mathrm{kDa})$ ions under typical quadrupole ion storage conditions (Goeringer \& McLuckey, 1998) have particular relevance to the issue of fragmentation as a result of ion/ion reactions. Two models were used that are expected to bracket the range of cooling rates expected under normal ion-trap operating conditions. A diffuse scattering model (Chen et al., 1997), which makes the assumption that each ion/helium collision is highly efficient at removing excess internal energy from the ion, was used to determine the highest cooling rates that might be expected. Alternatively, the exponential model for inefficient colliders (Tardy \& Rabinovitch, 1977), which employs a relatively small energy down-step size, was used to estimate the lowest cooling rates that might be expected. For the multiple alanine-glycine (AG) polypeptide ions (Griffin \& McAdoo, 1993) modeled in the study [viz., $(\mathrm{AG})_{8}-(\mathrm{AG})_{32}$ ], the collisional cooling rates of $200-2000 \mathrm{~s}^{-1}$ that were obtained with the diffuse scattering model give cooling rates about a factor of three greater than the inefficient colliders model for the same ion. Rice-Ramsperger-Karplus-Marcus (RRKM) calculations were also conducted for these ions to determine unimolecular dissociation rates under the same energy conditions used to determine collisional cooling rates. Figure 25 compares plots of unimolecular dissociation rates, $\mathrm{k}_{\mathrm{uni}}$ (dashed line), and apparent cooling rates derived from the inefficient colliders model, $\mathrm{k}_{\text {app }}$ (solid line), for singly charged 


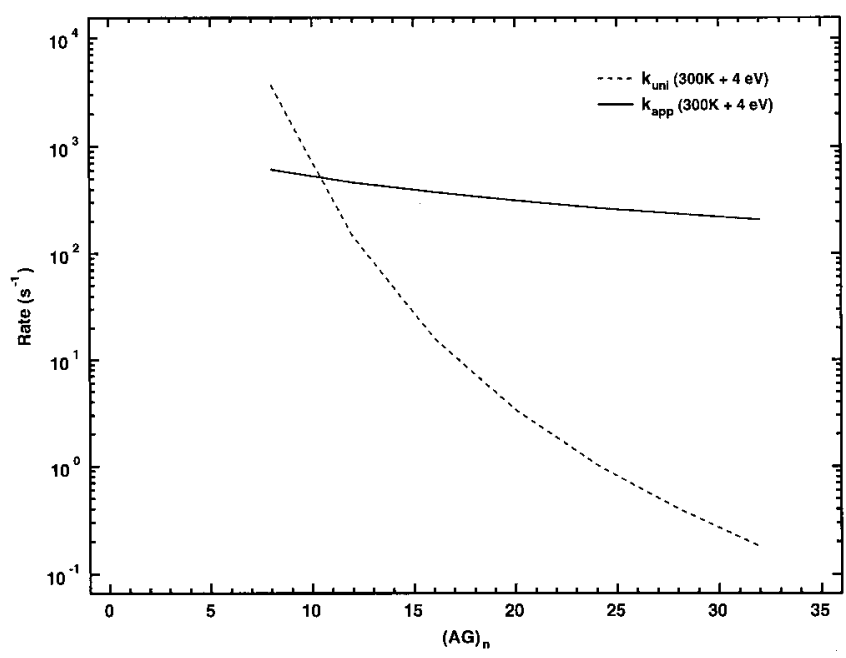

FIGURE 25. Comparison of the unimolecular dissociation rate, $\mathrm{j}_{\text {uni }}$, and the cooling rate, $\mathrm{k}_{\mathrm{app}}$, for $(\mathrm{AG})_{\mathrm{n}}$-mers that have an internal energy equal to the average internal energy at $300 \mathrm{~K}$ plus $4 \mathrm{eV}$. (Reprinted from the Int J Mass Spectrom, to appear, with kind permission of Elsevier Science-NL, Sara Burgerhartstrasse 25, $1055 \mathrm{KV}$, Amsterdam, The Netherlands.)

$(\mathrm{AG})_{8}-(\mathrm{AG})_{32}$ ions that have an internal energy that is equal to the average internal energy at $300 \mathrm{~K}$ plus $4 \mathrm{eV}$. The value of $4 \mathrm{eV}$ was chosen to correspond in energy to a $308-\mathrm{nm}$ photon. However, this simulation applies to any situation in which an ion experiences a relatively rapid input of internal energy, such as might occur in an ion/ion reaction. Note that apparent cooling rates decrease only marginally with ion size, whereas the unimolecular dissociation rates decrease by orders of magnitude in going from $(\mathrm{AG})_{8}$ to $(\mathrm{AG})_{32}$. For any system with a unimolecular dissociation rate less than the cooling rate, no fragmentation would be expected, because the excess internal energy would be removed before reaction can occur.

Even for ions with an initial $\mathrm{k}_{\text {uni }}$ somewhat greater than the cooling rate, the competing process of collisional cooling can prevent the dissociation of some ions that might otherwise dissociate. This situation is illustrated in Fig. 26 with a random walk simulation of $(\mathrm{AG})_{8}$ ions formed initially with average energy at $300 \mathrm{~K}$ plus $4 \mathrm{eV}$ in the presence of $1 \mathrm{mtorr}$ helium, which includes dissociation and ion cooling. Figure 26 shows a plot of the fraction of undissociated $(\mathrm{AG})_{8}$ ions (diamonds) and the fraction of ion internal energy that remains in the $(\mathrm{AG})_{8}$ ions (solid line) as a function of time. At $\mathrm{t}=0, \mathrm{k}_{\text {uni }}$ is $3700 \mathrm{~s}^{-1}$, and $\mathrm{k}_{\text {app }}=610 \mathrm{~s}^{-1}$. By $100 \mu \mathrm{s}, \mathrm{k}_{\text {uni }}$ drops to $1800 \mathrm{~s}^{-1}$ as a result of energy lost via collisions and, by $500 \mu \mathrm{s}, \mathrm{k}_{\text {uni }}$ drops to $790 \mathrm{~s}^{-1}$. By $1 \mathrm{~ms}$, virtually all ions that will fragment have done so, over $50 \%$ of the initial $(A G)_{8}$ ion population is left intact. In the absence of a cooling mechanism, only $2-3 \%$ of the initial ion population would survive to $1 \mathrm{~ms}$. This simulation illustrates how ion/ bath gas collisions can suppress the fragmentation of excited ions that are formed by ion/ion reactions. As a general rule, if fragmentation rates of a high-mass ion, excited by a rapid input of internal energy, do not exceed ca. $10^{4} \mathrm{~s}^{-1}$, then the ion-cooling simulation studies suggest that $1 \mathrm{mtorr}$ helium at room temperature can prevent a significant number of the ions from dissociating. This effect is believed to be an important factor in the relative lack of dissociation associated with ion/ion reactions of relatively high-mass ions in the ion trap.

Relatively little in detail can be said about energy partitioning and its possible role in the extent of fragmentation of high-mass product ions that arise from charge transfer reactions, because the experiments reported to date provide no information on the translational energies of the products nor on the fate of the neutral product. However, the attachment reactions are enlightening in this regard. The attachment of iodide anions to protein cations provides a clear example of how an excited noncovalently bound complex can be stabilized before it dissociates, when all the reaction exothermicity is present in the complex. Figure 27 shows a hypothetical energy diagram for the attachment of $\mathrm{I}^{-}$to a multiply protonated protein. This diagram is qualitatively very similar to that shown for ion/ion proton transfer [Fig. 14 (top)], because the attachment product is known to be an intermediate for proton transfer. That is, the most facile fragmentation of the attachment product is loss of HI. Perhaps the major difference between the energy diagram associated with $(\mathrm{M}+\mathrm{nH})^{\mathrm{n}+} / \mathrm{I}^{-}$and those associated with $(\mathrm{M}+\mathrm{nH})^{\mathrm{n}+} / \mathrm{PDCH}$ anions (a combination that yields $100 \%$ proton transfer and no adduct formation) is the well-depth

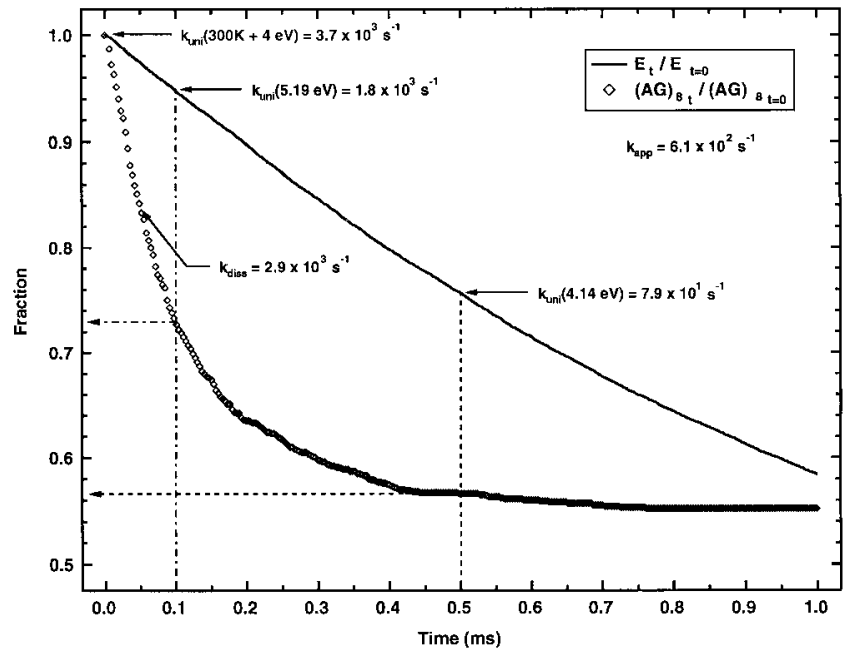

FIGURE 26. Composite cooling curve and corresponding fraction of surviving $(\mathrm{AG})_{8}$ ions as a function of time, following the input of $4 \mathrm{eV}$ into a $300 \mathrm{~K}$ ion. The phenomenologic cooling rate, $\mathrm{k}_{\text {app }}$, is $610 \mathrm{~s}^{-1}$ and the observed dissociation rate, $\mathrm{k}_{\text {diss }}$, is $2900 \mathrm{~s}^{-1}$. Unimolecular dissociation rates, $\mathrm{k}_{\mathrm{uni}}(\mathrm{E})$ are also indicated for the initial $\mathrm{E}(300 \mathrm{~K}$ ion plus $4 \mathrm{eV})$ and for energies at cooling times of $100(5.19 \mathrm{eV})$ and $500(4.14 \mathrm{eV}) \mu \mathrm{s}$. (Reprinted from the Int J Mass Spectrom 1998, to appear, with kind permission of Elsevier Science-NL, Sara Burgerhartstrasse 25, $1055 \mathrm{KV}$, Amsterdam, The Netherlands.) 


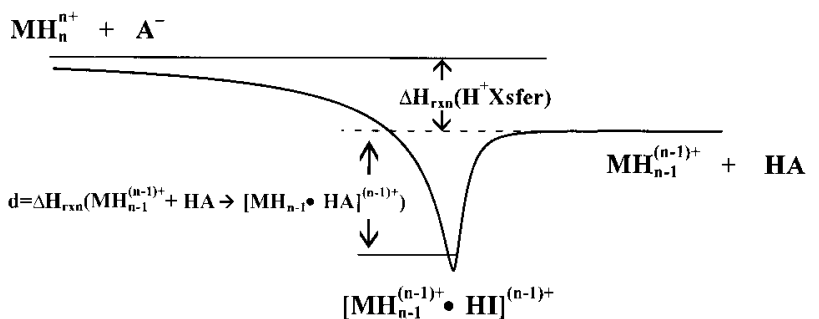

FIGURE 27. Hypothetical energy diagram for ion/ion proton transfer from a multiply protonated protein, $(\mathrm{M}+\mathrm{nH})^{\mathrm{n}+}$, to $\mathrm{I}^{-}$. (Reprinted with permission from J Am Chem Soc 1997, 119, 1688-1696, copyright 1997 American Chemical Society.)

(d) associated with the adduct. Deeper wells lead to greater kinetic stability of the proton transfer intermediate and to a greater likelihood for collisional stabilization of the intermediate before it can proceed to products. Cation and anion attachment reactions demonstrate unambiguously that collisional stabilization of the intermediate can occur even when all the ion/ion reaction exothermicity is present when $1 \mathrm{mtorr}$ of helium bath gas is present.

\section{APPLICATIONS}

Ion chemistry has long played an important role in applications of mass spectrometry. Although high-mass, multiply charged ion chemistry research is still a relatively new area, unimolecular dissociation of high-mass ions has already proved to be useful, and is widely employed in providing primary structure information on polymeric species. Ion/molecule and ion/ion chemistry can also play useful roles in mass spectrometry applications, particularly in manipulating charge states of multiply charged ions. Based on what has already been learned about proton transfer chemistry that involves multiply protonated molecules in the gas-phase, charge-state manipulation appears to be a role that is particularly well-suited for ion/ion chemistry. Another possible role might be in the chemical modification of ions in the gas phase. This goal might be desirable if such a modification were to alter the unimolecular chemistry of an ion; for example, useful structural information about the precursor molecule can be obtained from the modified ion that is not accessible from the unmodified ion. An example of such an approach with ion/molecule chemistry, alluded to in the Introduction, has been described whereby oligonucleotide anions modified by attachment of a trimethylsilyl group to a phosphodiester linkage yielded more structural information upon MS/MS than did the unmodified anions (O'Hair \& McLuckey, 1997).

The formation of novel ions that are difficult to make otherwise, such as the formation of radical anions of oligonucleotides via ion/ion electron transfer chemistry, might also qualify as a "chemical modification." The radical anions have shown unique unimolecular dissociation reactions, although such reactions have thus far not proved to yield additional structural information. The possible chemical modification of gaseous ions by ion/ion chemistry to this point has been little explored, and no further mention of it will be made here. Most emphasis has been placed on the use of ion/ion chemistry for charge state manipulation. The remainder of this section, therefore, summarizes research performed to date intended to evaluate the potential for ion/ion chemistry in various experiments where charge-state manipulation may be desirable.

\section{A. Electrospray Mixture Analysis}

The mixture complexity that electrospray can deal with directly is limited, in some cases by matrix effects on ionization, and, in others, by the "spectral congestion" that arises from extensive overlap in mass-to-charge by ions that have different masses and charges. The latter limitation can be ameliorated somewhat by use of algorithms intended to convert mass-to-charge spectra to "zero charge" mass spectra (Mann et al., 1989; Ferrige et al., 1991; Reinhold and Reinhold, 1992; Labowsky et al., 1993; Zhang et al., 1998). Nevertheless, as spectral complexity and chemical noise increase, the algorithms become increasing prone to yield artifact peaks and to miss mixture components that give rise to small signals. Means for simplifying electrospray mass spectra that are complicated by the multiple-charging phenomenon by means such as ion/ion chemistry are, therefore, of interest. The high exothermicity of ion/ion reactions, the charge-squared dependence of the kinetics, and the minimal degree of fragmentation that result from most types of ion/ ion reactions make them particularly promising as a means to simplify complex electrospray mass spectra.

For the use of ion/ion reactions in mixture analysis, there are particular figures of merit associated with the reactant used to reduce charge. They are:

(i) Well-defined ion/ion chemistry. This criterion implies that a single reaction leading to charge reduction takes place for each and every charge state. Ideally, this situation leads to a final charge state ion population that is as homogeneous in mass as possible. For example, in the case of cations, the final product ion may be the singly protonated molecule. A reactant that engages in competing reactions, such as proton transfer and anion/cation attachment, is undesirable, because it can complicate the mixture. Fragmentation is also highly undesirable, because it can lead to very complex spectra that are difficult to interpret. However, as discussed above, fragmentation is only observed for highly exothermic reactions (e.g., electron 
transfer to rare gas cations) that involve relatively small multiply charged ions.

(ii) Minimal ion/neutral effects. In cases where the neutral species from which the reactant ion is derived is present at all times, it is important that the neutral species have minimal deleterious effects on the analysis. Examples of such effects include ion/ molecule reactions that might complicate the mixture.

(iii) High number density. The highest rates of reaction are obtained when the singly charged reactant is in great excess over the multiply charged reactant. Reaction rates then follow pseudo-first-order kinetics, and the rates are proportional to the square of the product of the charges of the reactants. It is, therefore, highly desirable to use as large a population of singly charged ions as possible to minimize the reaction time required to reduce bio-ion charge states to the intended level.

(iv) High mass-to-charge. This figure of merit is particular to the ion-trap experiment, because the limit to which charges can be reduced is usually determined by the limit to which ions of opposite polarity and widely different mass-to-charge can be stored simultaneously. As a result of a limited dynamic mass-to-charge storage range, it is, therefore, desirable that the reactant singly charged ion be as high in mass-to-charge as possible, so that high trapping amplitudes can be used to provide a high upper limit to mass-to-charge. Some of these points are amplified further in the following sections, which describe mixture analysis data derived from polypeptides, oligonucleotides, and poly(ethylene glycols).

\section{Protein/Peptide Mixtures}

The major application of ion/ion chemistry in electrospray mixture analysis has been in the area of peptide and protein analysis (Stephenson \& McLuckey, 1996b; Stephenson \& McLuckey, 1998a; McLuckey et al., 1998). This research has involved positive ion electrospray and anions derived from PDCH. Positive ions were largely either singly or multiply protonated. This situation illustrates the optimal case whereby the singly charged positive ions of each component are the $(\mathrm{M}+\mathrm{H})^{+}$species. For a protein initially present as a mixture of charge states that arise from multiple protonation, the final product for the set of consecutive proton transfer reactions that lead to a singly charged ion is the same regardless of initial charge state, as indicated below:

$$
(\mathrm{M}+n \mathrm{H})^{n+}+(n-1) \mathrm{Y}^{-} \rightarrow(\mathrm{M}+\mathrm{H})^{+}+(n-1) H Y
$$

$$
\begin{aligned}
&(\mathrm{M}+(n-1) \mathrm{H})^{(n-1)+}+(n-2) \mathrm{Y}^{-} \rightarrow(\mathrm{M}+\mathrm{H})^{+}+(n-2) \mathrm{HY} \\
&(\mathrm{M}+(n-2) \mathrm{H})^{(n-2)+}+(n-3) \mathrm{Y}^{-} \rightarrow \\
&(\mathrm{M}+\mathrm{H})^{+}+(n-3) \mathrm{HY}
\end{aligned}
$$

etc.

Anion attachment, on the other hand, although as effective as proton transfer in reducing the charges of multiply protonated proteins, leads to a family of singly charged products the complexity of which is determined by the range of initial charge states; i.e.,

$$
\begin{gathered}
(\mathrm{M}+n \mathrm{H})^{n+}+(n-1) \mathrm{Y}^{-} \rightarrow(\mathrm{M}+n \mathrm{H}+(n-1) \mathrm{Y})^{+} \\
(\mathrm{M}+(n-1) \mathrm{H})^{(n-1)+}+(n-2) \mathrm{Y}^{-} \rightarrow \\
(\mathrm{M}+(n-1) \mathrm{H}+(n-2) \mathrm{Y})^{+} \\
(\mathrm{M}+(n-2) \mathrm{H})^{(n-2)+}+(n-3) \mathrm{Y}^{-} \rightarrow \\
(\mathrm{M}+(n-2) \mathrm{H}+(n-3) \mathrm{Y})^{+} \quad(20) \\
\text { etc. }
\end{gathered}
$$

For the current ion-trap arrangement of Fig. 3, the most successful mixture analysis applications are those whereby the mixture components are positively charged protonated species, and proton transfer to anions of perfluorocarbons is the most appropriate ion/ion reaction for charge-state reduction.

Recent analyses of several peptide/protein mixtures of known composition (Stephenson \& McLuckey, 1998a), including a 45-component mixture described below, illustrate the use of ion/ion proton transfer chemistry in electrospray mixture analysis. Table 1 summarizes the list of components in the 45-member mixture, their nominal molecular weights, and their nominal solution concentration. Figure 28 shows the ES mass spectrum of the mixture of Table 1, which is comprised of peptides and proteins that range in mass from ca. 0.5-100 kDa. Almost all the electrospray signal fell within the mass-to-charge range plotted in the figure. This spectrum is far too complex to interpret in a reliable fashion, either manually or with an algorithm to transform the data to a "zero-charge" spectrum. This example illustrates the high degree of spectral congestion that can result from the electrospray of biopolymer mixtures.

Figures 29(a)-(d) show the data recorded after the cations of Fig. 28 were subjected to ion/ion proton transfer reactions with the $(\mathrm{M}-\mathrm{F})^{-}$and $\left(\mathrm{M}-\mathrm{CF}_{3}\right)^{-}$anions derived from glow discharge ionization of $\mathrm{PDCH}$ for $70 \mathrm{~ms}$. Note that the ion/ion reactions dramatically expand the mass-tocharge scale over which the cations are observed. Although the data are plotted over segments of the total mass-to- 
TABLE 1. Oligopeptide mixture components for data of figures 28 and 29

\begin{tabular}{|c|c|c|c|c|c|c|c|}
\hline Name & $M^{\mathrm{a}}$ & $\#^{\mathrm{b}}$ & $C^{\mathrm{c}}$ & Name & $M^{\mathrm{a}}$ & $\#^{\mathrm{b}}$ & $C^{\mathrm{c}}$ \\
\hline Leucine enkephalin & 555 & 1 & 18.0 & $\alpha$-endorphin & 1746 & 23 & 17.6 \\
\hline Laminin fragment & 595 & 2 & 16.8 & Phospho pep II & 1835 & 24 & 16.4 \\
\hline AA peptide & 910 & 3 & 20.8 & Dynorphin A & 2147 & 25 & 23.2 \\
\hline Tryptic peptide V & 922 & 4 & 20.0 & Melittin & 2847 & 27 & 17.5 \\
\hline Xenopsin & 980 & 5 & 18.2 & Glucagon & 3483 & 28 & 14.4 \\
\hline ac-K peptide & 1009 & 6 & 19.8 & Bovine insulin & 5737 & 29 & 10.9 \\
\hline ac-H peptide & 1018 & 7 & 19.6 & BPTI & 6512 & 31 & 15.3 \\
\hline ac-R peptide & 1037 & 8 & 19.2 & Bovine ubiquitin & 8565 & 33 & 14.8 \\
\hline Tryptic peptide $\mathrm{P}$ & 1037 & 9 & 20.0 & Cytochr. $c$ (tuna) & 12170 & 35 & 5.0 \\
\hline Angiotensin II & 1046 & 10 & 19.1 & Cytochr. $c$ (bov.) & 12327 & 36 & 8.8 \\
\hline Bradykinin & 1060 & 11 & 18.8 & Cytochr. $c$ (horse) & 12384 & 37 & 4.5 \\
\hline Bov. ins., (B-f22-30) & 1086 & 12 & 18.4 & Ribonucl. A (eq.) & 13682 & 38 & 5.1 \\
\hline Neuromedin U-8 & 1111 & 13 & 18.6 & $\alpha$-lactalbumin & 14178 & 39 & 4.3 \\
\hline Dephospho pep I & 1130 & 14 & 17.4 & Lysozyme (chicken) & 14305 & 40 & 2.3 \\
\hline Gramacidin S & 1142 & 15 & 18.0 & Apomyoglobin & 16950 & 42 & 3.7 \\
\hline LHRH & 1182 & 16 & 16.8 & $\beta$-lactoglobulin B & 18277 & 43 & 5.4 \\
\hline Phospho pep I & 1211 & 17 & 16.4 & $\beta$-lactoglobulin A & 18363 & 44 & 5.4 \\
\hline Angiotensin I & 1296 & 18 & 14.4 & Carbonic anhyd. & 29219 & 45 & 3.4 \\
\hline Dynorphin B & 1570 & 19 & 15.9 & Ovalbumin & $\approx 45 \mathrm{k}$ & 46 & 2.4 \\
\hline Somatosin & 1637 & 20 & 12.2 & Bovine albumin & 66462 & 47 & 2.9 \\
\hline Neurotensin & 1673 & 21 & 16.1 & Conalbumin (chick.) & 76244 & 48 & 2.2 \\
\hline \multirow[t]{2}{*}{ Dephospho pep II } & 1674 & 22 & 17.9 & Transferrin (bovine) & 78030 & 49 & 2.3 \\
\hline & & & & Phosphorylase $\mathrm{B}^{\mathrm{d}}$ & $\approx 139 \mathrm{k}$ & 50 & 2.8 \\
\hline
\end{tabular}

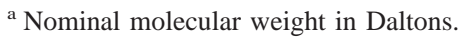

${ }^{\mathrm{b}}$ Arbitrary number assigned to component for figure annotation purposes.

${ }^{c}$ Nominal concentration in mixture in units of $\mu \mathrm{M}$.

${ }^{\mathrm{d}}$ The ES data indicated a protein of molecular weight of roughly $97500 \mathrm{Da}$.

charge scale to facilitate visual inspection, it is important to recognize that all data were collected under a fixed set of ion/ion reaction conditions (viz., the same number of anions and the same reaction time). The data of Figs. 29(a)-(d) are dominated by ions with charges of +1 to +3 . Not surpris-

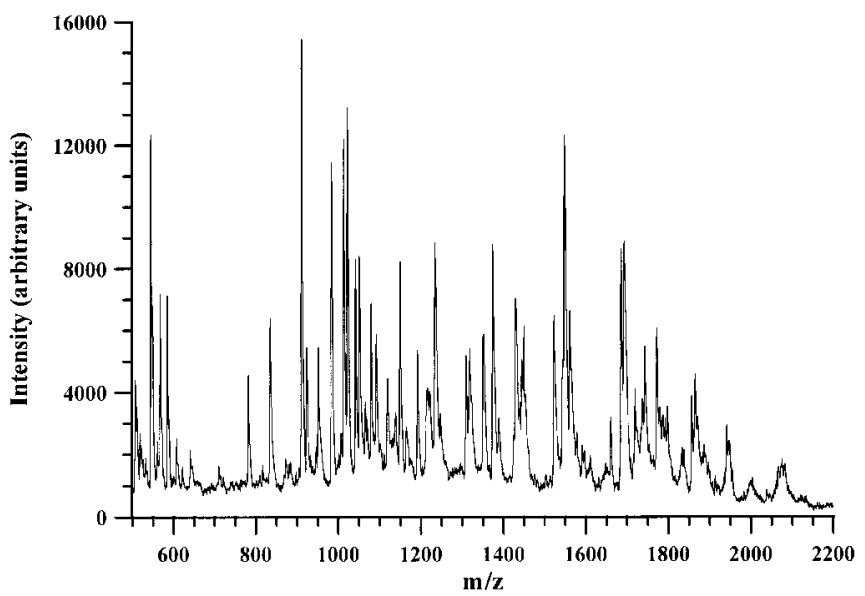

FIGURE 28. Positive ion electrospray mass spectrum obtained for the mixture comprised of the components listed in Table 1. (Reprinted from the J Am Soc Mass Spectrom 1998, 9, 585-596, with permission from Elsevier Science, Inc. Copyright 1998 by the American Society for Mass Spectrometry.) ingly, singly charged ions are most abundant for lower mass mixture components (up to bovine insulin), whereas doubly charged ions tend to be most abundant for the intermediate mass species (components 31-45). The largest observed proteins tend to show triply charged ions of an abundance comparable to or greater than the doubly charged ions. At somewhat longer reaction times (ca. $100 \mathrm{~ms}$ ), the most abundant ions from all species can be driven to the +1 charge state. For the purposes of this particular study, however, an ion/ion reaction period of a duration sufficient to retain a significant number of doubly and triply charged ions from the highest mass components was used so that all the ions fell below a mass-to-charge of 40,000. Signals that can be associated with at least 42 of the known components [and several unknown components, such as those associated with the peaks labeled as (A) and (B) in Fig. 29(b)] can be identified. The components that cannot be located in the mass spectrum are those that yielded quite small signals, even when they were individually subjected to electrospray. These components include ovalbumin, and the singly charged ions from carbonic anhydrase and ribonuclease A. [Singly charged ions of ribonuclease A were observed after the ion/ion reaction experiment was performed on a 5:1 dilution of the mixture (data not shown).] In fact, virtually all the signals were similar to the relative ES responses obtained 

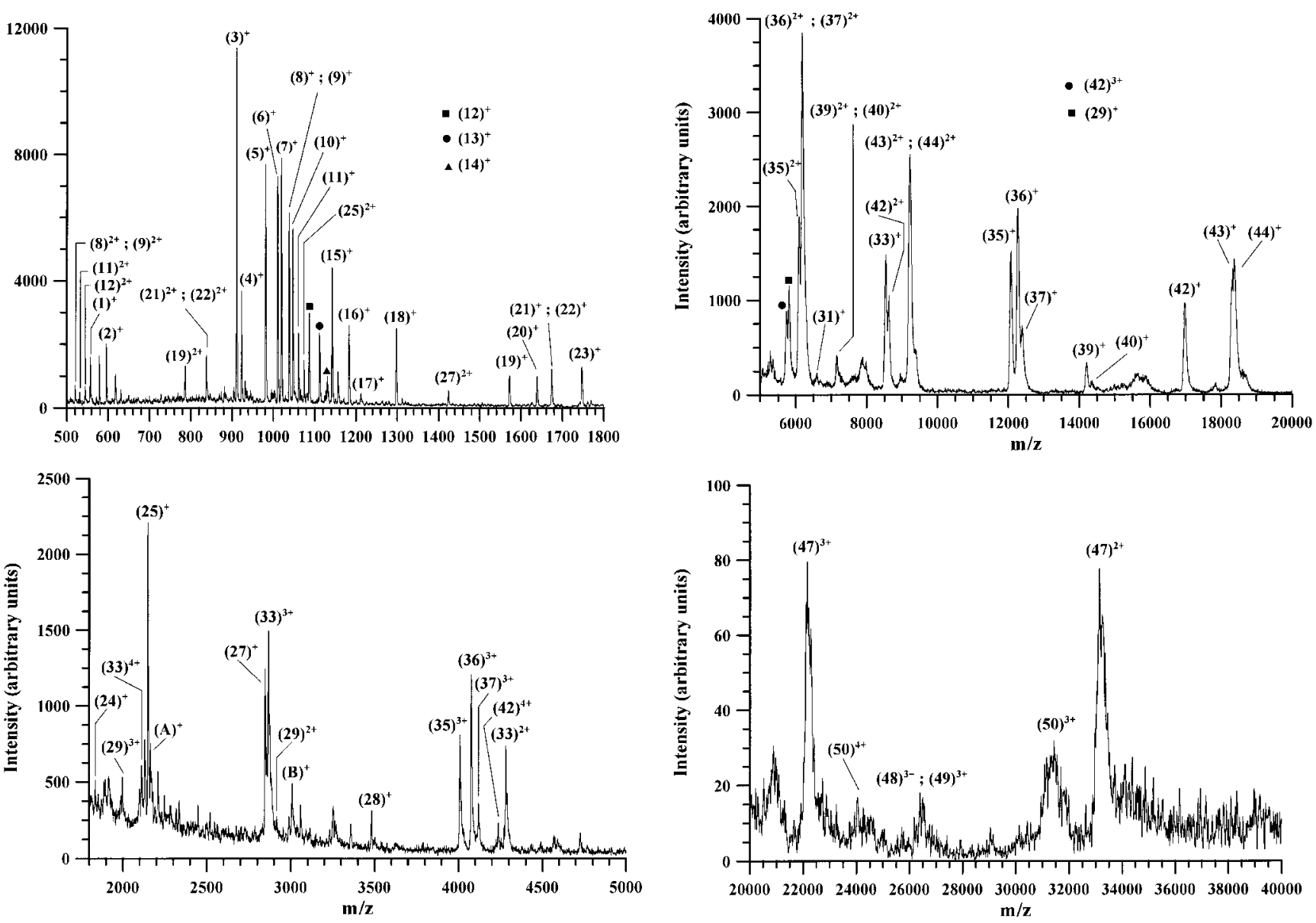

FIGURE 29. Electrospray mass spectra of the mixture of ions reflected in Fig. 28 after being subjected to ion/ion proton transfer reactions for $70 \mathrm{~ms}$ : (a) $\mathrm{m} / \mathrm{z} 500-1,800$, (b) $\mathrm{m} / \mathrm{z} 1,800-5,000$, (c) $\mathrm{m} / \mathrm{z} 5,000-20,000$, and (d) $m / z, 20,000-40,000$. (Reprinted from the J Am Soc Mass Spectrom 1998, 9, 585-596, with permission from Elsevier Science, Inc. Copyright 1998 by the American Society for Mass Spectrometry.)

with the various components when they were subjected to ES in the absence of the other components. The only indications of a possible "matrix effect" were the diminished signals of the three relatively high mass and poorly responding proteins. Such an observation might result from a matrix effect upon ionization, or upon a differential ion storage effect in the ion trap, whereby ions of high mass-to-charge are preferentially lost as a result of scattering due to collisions of ions of like charge. In any case, these results demonstrate that a fairly complex mixture of polypeptides with a wide range of masses, electrospray responses, and, presumably, gas-phase conformations, can be analyzed by electrospray combined with ion/ion chemistry.

\section{Nucleic Acid Mixtures}

Negative ion electrospray is generally used for oligonucleotides and for mononucleotides as a result of their high acidities in solution (Nordhoff et al., 1996; Limbach, 1996).
They are generally observed in electrospray mass spectra as deprotonated species, although they are often comprised of mixtures of proton and sodium counter-ions. As with the opposite polarity combination, proton transfer is the best ion/ion reaction for reducing charge states of deprotonated species to yield the simplest spectrum at low charge states; i.e.:

$$
\begin{gathered}
(\mathrm{M}-n \mathrm{H})^{n-}+(n-1) \mathrm{YH}^{+} \rightarrow(\mathrm{M}-\mathrm{H})^{-}+(n-1) \mathrm{Y} \\
(\mathrm{M}-(n-1) \mathrm{H})^{(n-1)-}+(n-2) \mathrm{YH}^{+} \rightarrow(\mathrm{M}-\mathrm{H})^{-}+(n-2) \mathrm{Y} \\
(\mathrm{M}-(n-2) H)^{(n-2)-}+(n-3) \mathrm{YH}^{+} \rightarrow(\mathrm{M}-\mathrm{H})^{-}+(n
\end{gathered}
$$

etc.

Little attention thus far has been placed on nucleic acid 
mixture analysis by using ion/ion proton transfer to multiply deprotonated nucleic acids due to limitations in producing high abundances of high-mass protonated molecules using the instruments of Figs. 2 and 3.

Cation attachment to nucleic acid anions is clearly not an attractive reaction for mixture analysis, because it leads to a complex set of products at low charge; i.e.:

$$
\begin{gathered}
(\mathrm{M}-n \mathrm{H})^{n-}+(n-1) \mathrm{Y}^{+} \rightarrow(\mathrm{M}-n \mathrm{H}+(n-1) \mathrm{Y})^{-} \\
(\mathrm{M}+(n-1) \mathrm{H})^{(n-1)-}+(n-2) \mathrm{Y}^{+} \rightarrow \\
(\mathrm{M}-(n-1) \mathrm{H}+(n-2) \mathrm{Y})^{-} \\
(\mathrm{M}+(n-2) \mathrm{H})^{(n-2)-}+(n-3) \mathrm{Y}^{+} \rightarrow \\
(\mathrm{M}-(n-2) \mathrm{H}+(n-3) \mathrm{Y})^{-}(22) \\
\text { etc. }
\end{gathered}
$$

Likewise, electron transfer leads to a mixture of singly charged products; i.e.:

$$
(\mathrm{M}-n \mathrm{H})^{n-}+(n-1) \mathrm{Y}^{+} \rightarrow(\mathrm{M}-n \mathrm{H}-(n-1) e-)^{-}+(n
$$

$$
\begin{gathered}
(\mathrm{M}-(n-1) \mathrm{H})^{(n-1)-}+(n-2) \mathrm{Y}^{+} \rightarrow \\
(\mathrm{M}-(n-1) \mathrm{H}-(n-2) e-)^{-}+(n-2) \mathrm{Y} \\
(\mathrm{M}-(n-2) \mathrm{H})^{(n-2)+}+(n-3) \mathrm{Y}^{+} \rightarrow \\
(\mathrm{M}-(n-2) \mathrm{H})-(n-3) e-)^{-}+(n-3) \mathrm{Y} \quad(23)
\end{gathered}
$$

etc.

In the case of electron transfer to multiply deprotonated species, the components making up the mixture of singly charged products are spaced by $1 \mathrm{Da}$. This situation is illustrated in Fig. 30, which shows an overlay of mass spectra plotted over the $m / z$ range of the -5 charge state of $5^{\prime}-\mathrm{d}$ (GTCTTAGCGCTAAGAC)-3'. The filled circles that show the peaks produced directly by electrospray indicate the ions $(\mathrm{M}-5 \mathrm{H})^{5-}$, $(\mathrm{M}-6 \mathrm{H}+\mathrm{Na})^{5-}$, and $(\mathrm{M}-7 \mathrm{H}+2 \mathrm{Na})^{5-}$. The filled squares indicate the peaks produced by electron transfer reactions to the -8 precursor ions produced by electrospray. In this case, the -5 product ions correspond to $\left(\mathrm{M}-8 \mathrm{H}-3 \mathrm{e}^{-}\right)^{5-}$, $\left(\mathrm{M}-9 \mathrm{H}+\mathrm{Na}-3 \mathrm{e}^{-}\right)^{5-}$, and $\left(\mathrm{M}-10 \mathrm{H}+2 \mathrm{Na}-3 \mathrm{e}^{-}\right)^{5-}$. Proton transfer to the -8 ions, on the other hand, would be expected to yield the same nominal ions as derived directly from electrospray. This comparison highlights the problem with the use of electron transfer to deprotonated molecules to manipulate charge for the purpose of mixture analysis, because a mixture of precursor ion masses that results from different numbers of missing protons from the same molecule results in the same nominal mixture of product ion masses (less the mass of one or more electrons).

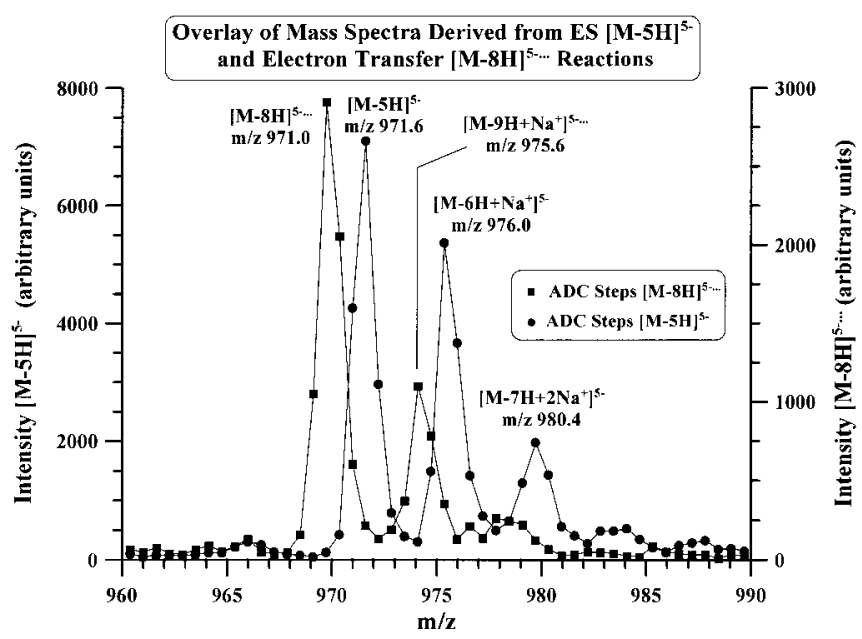

FIGURE 30. Overlay of negative ion electrospray mass spectra over the $\mathrm{m} / \mathrm{z}$ range 960-990 derived directly from electrospray of $5^{\prime}$-d(GTCTTAGCGCTAAGAC)-3' (filled circles) and from electron transfer from the $(\mathrm{M}-8 \mathrm{H})^{8-}$ and $(\mathrm{M}-9 \mathrm{H}+\mathrm{Na})^{8-}$ ions to xenon cations (filled squares). (Reprinted from Rapid Commun Mass Spectrom 1997, 11, 875-880 with permission. Copyright 1997 John Wiley \& Sons, Ltd.)

Another possibility for oligonucleotide mixture analysis might be to use the same polarity combination used for peptides and proteins, viz., multiply charged positive ions/ perfluorocarbon anions. Several reports have recently appeared in which electrospray conditions for ionizing oligonucleotides in the positive ion mode have been reported (Sannes-Lowery et al., 1997; Ni et al., 1997; Wang et al., 1997). These conditions lead to multiply protonated oligonucleotides, whereby all the phosphodiester linkages are neutralized and the protons presumably reside on nucleobases. Figure 31 indicates that positive ion oligonucleotide

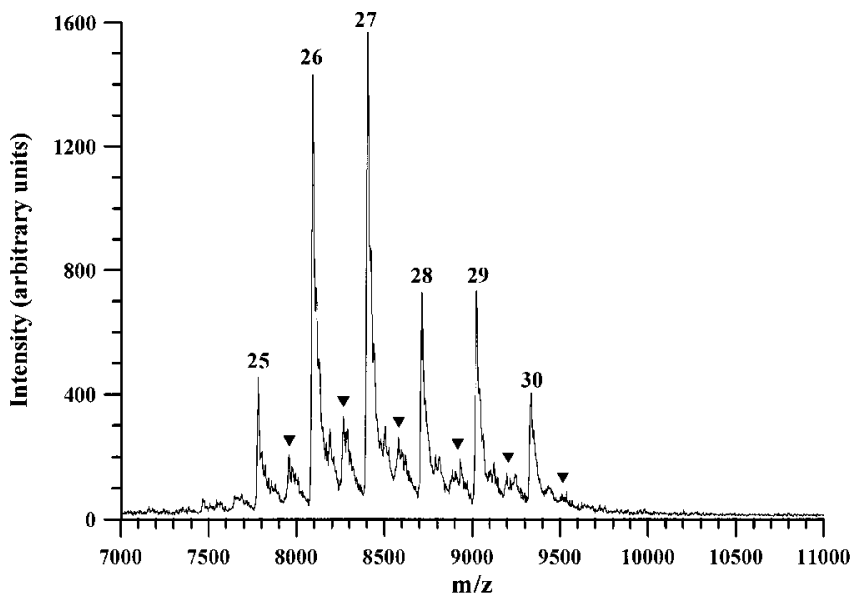

FIGURE 31 . Post-ion/ion reaction positive ion spectrum obtained after a 25-30-mer mixture of $5^{\prime}-\mathrm{d}(\mathrm{A})_{\mathrm{n}}-3^{\prime}$ positive ions was subjected to reaction with anions derived from PDCH. (Peaks indicated with inverted filled triangles are unknown mixture components that were also noted in the pre-ion/ion mass spectrum.) 
mixtures behave very similarly to positive ion polypeptide mixtures in reactions with anions derived from PDCH. This spectrum shows the singly charged product ions, $(\mathrm{M}+\mathrm{H})^{+}$, where $\mathrm{M}=5^{\prime}-\mathrm{d}(\mathrm{A})_{25}-3^{\prime}$ to $5^{\prime}-\mathrm{d}(\mathrm{A})_{30}-3^{\prime}$, from ion/ion proton transfer reactions that result from positive ion electrospray of the nucleic acids and negative ion glow discharge of PDCH. As with other moderately large polymeric species, such as peptides and proteins, little or no evidence for dissociation of the oligonucleotides as a result of ion/ion proton transfer could be identified in this experiment. There appears to be no limitation imposed by ion/ion chemistry for electrospray mixture analysis of nucleic acids in the positive ion mode.

\section{Poly(ethylene glycols)}

Synthetic polymers constitute a large group of species for which ion/ion chemistry in combination with electrospray may find use. (For a recent review of the application of electrospray to nonbiological polymers, see Saf et al., 1997). The multiple-charging phenomenon can pose particularly troublesome problems in determining molecular weight distributions, repeat units, end groups, etc., when it gives rise to significant overlap in mass and charge distributions. By far, most recent attention has been directed toward the application of matrix-assisted laser desorption to synthetic polymers, in part to avoid complications of multiple-charging (Montaudo, 1996). One approach to addressing the mass and charge state overlap that is present in electrospray mass spectra is to apply high resolving power, as has been demonstrated by use of Fourier transform ion cyclotron resonance (O'Connor \& McLafferty, 1995). Another approach might be to collapse the charge state distribution to the singly charged ions.

A recent report has described the ion/ion reactions that involved multiply charged PEGs formed via multiple sodium ion attachment (Stephenson \& McLuckey, 1998d). This study illustrates several important considerations in the use of ion/ion chemistry for electrospray mixture analysis. Figure 8 and its discussion (Section III.A.4) indicated that the high-mass PDCH anions reacted with $(\mathrm{M}+\mathrm{nNa})^{\mathrm{n}+}$ ions by fluoride transfer and the lower mass anions derived from $\mathrm{PDCH}$ reacted by proton abstraction. Fluoride transfer is not a particularly useful reaction for mixture analysis, because it leads to a complicated spectrum of singly charged products that consist of the normal polymer distribution with varying numbers of sodium and fluoride ions attached to them; i.e.:

$$
\begin{aligned}
&(\mathrm{M}+n \mathrm{Na})^{n+}+(n-1) \mathrm{ZY}^{-} \rightarrow(\mathrm{M}+n \mathrm{Na}+(n-1) \mathrm{Y})^{+}+\mathrm{Z} \\
&(\mathrm{M}+(n-1) \mathrm{Na})^{(n-1)+}+(n-2) \mathrm{ZY}^{-} \rightarrow \\
&(\mathrm{M}+(n-1) \mathrm{Na}+(n-2) \mathrm{Y})^{+}+\mathrm{Z}
\end{aligned}
$$

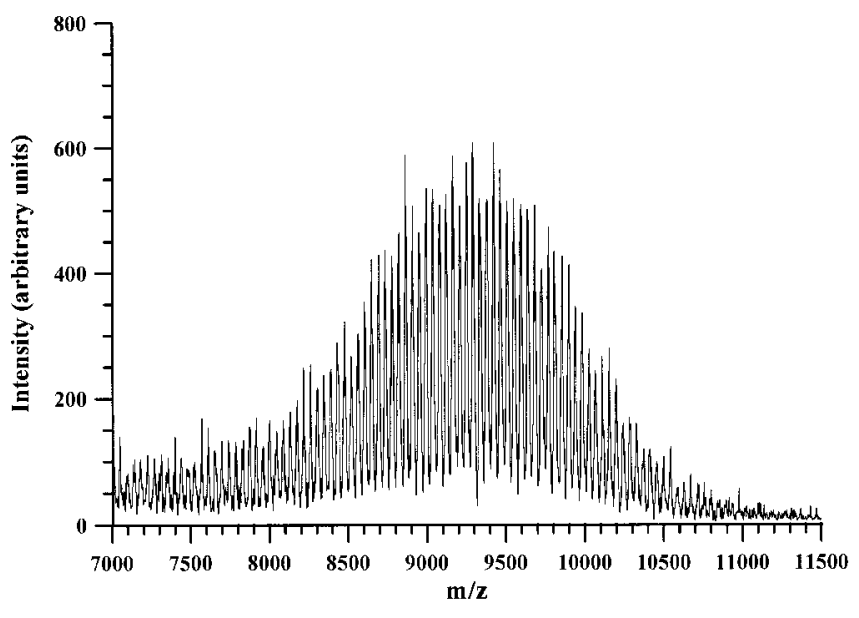

FIGURE 32. Positive ion electrospray mass spectrum that shows the +1 ions of PEG 8000 that result from ion/ion reactions with the $\left(\mathrm{M}-\mathrm{F}^{*}\right)^{-}$ion from perfluoro(methyldecalin). (Reprinted from the J Am Soc Mass Spectrom 1998, 9, 957-965, with permission from Elsevier Science, Inc. Copyright 1998 by the American Society for Mass Spectrometry.)

$$
\begin{gathered}
(\mathrm{M}+(n-2) \mathrm{Na})^{(n-2)+}+(n-3) \mathrm{ZY}^{-} \rightarrow \\
(\mathrm{M}+(n-2) \mathrm{Na}+(n-3) \mathrm{Y})^{+}+\mathrm{Z} \\
\text { etc. }
\end{gathered}
$$

Figure 32 shows the singly charged products that result from the positive ions derived from electrospray of a PEG 8000 sample in reactions with $\left(\mathrm{M}-\mathrm{F}^{\circ}\right)^{-}$ions derived from perfluoro(methyldecalin). These anions react with PEG cations exclusively by $\mathrm{F}^{-}$transfer. The singly charged product ion spectrum shows a distribution of signals that does not reflect accurately the molecular weight distribution of the PEG mixture. The products are comprised of single mixture components with various combinations of sodium cations and fluoride anions with sodium/fluoride ratios of $n /(n-1)$, where $\mathrm{n}$ is the value of an initial precursor ion charge state. This situation is not obvious at first glance, because the sum of the masses of sodium cation and fluoride (42 Da) is almost the same as the oligomer mass spacing for PEG (44 Da), and the spectrum was not acquired with a resolution sufficient to make apparent the mixture of products. The PEG molecular weight distribution, assuming (unrealistically) no other systematic discrimination effects in the experiment, could only be extracted from Fig. 32 if the values and relative abundances of the various initial charge states of the PEG ions were known.

The other major reaction mechanism noted for PEG $(\mathrm{M}+\mathrm{nNa})^{\mathrm{n}+}$ ions with perfluorocarbon anions was proton transfer to the anion. Like fluoride transfer, this reaction is problematic for mixture analysis, because it leads to products with different numbers of sodium ions; i.e.: 


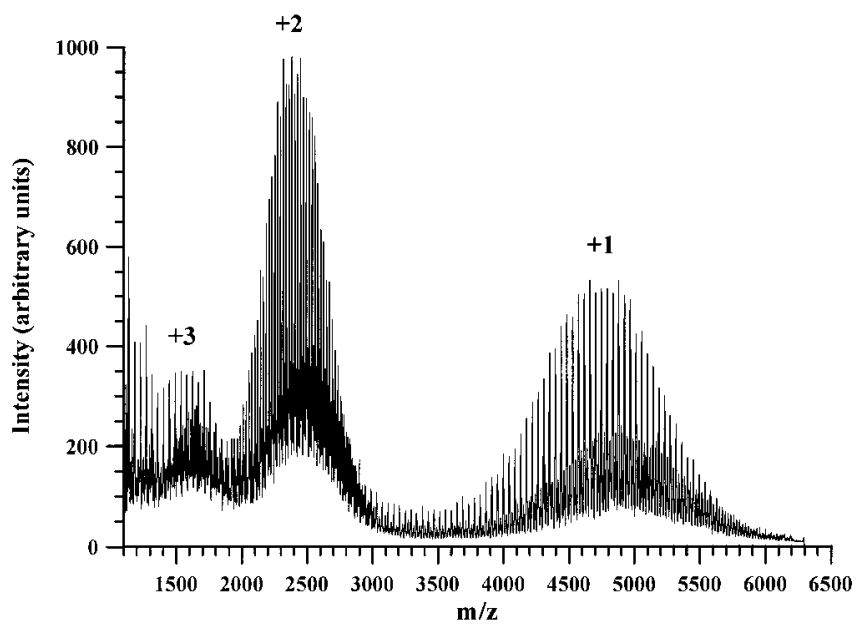

FIGURE 33. Positive ion electrospray mass spectrum obtained after cations formed from PEG 4600 were subjected to reaction with the $\left(\mathrm{M}-\mathrm{F}^{*}\right)^{-},\left(\mathrm{M}-\mathrm{CF}_{3 .}\right)^{-},\left(\mathrm{M}-\mathrm{C}_{4} \mathrm{~F}_{9 .}\right)^{-}$, and $\left(\mathrm{M}-\mathrm{C}_{5} \mathrm{~F}_{9 .}\right)^{-}$anions from PDCH for $143 \mathrm{~ms}$. (Reprinted from the J Am Soc Mass Spectrom 1998, 9, 957-965, with permission from Elsevier Science, Inc. Copyright 1998 by the American Society for Mass Spectrometry.)

$$
\begin{gathered}
(\mathrm{M}+n \mathrm{X})^{n+}+(n-1) \mathrm{Y}^{-} \rightarrow \\
(M-(n-1) \mathrm{H}+n \mathrm{X})^{+}+(n-1) \mathrm{HY} \\
(\mathrm{M}+(n-1) \mathrm{X})^{(n-1)+}+(n-2) \mathrm{Y}^{-} \rightarrow \\
(\mathrm{M}-(n-2) \mathrm{H}+(n-1) \mathrm{X})^{+}+(n-2) \mathrm{HY} \\
(\mathrm{M}+(n-2) \mathrm{X})^{(n-2)+}+(n-3) \mathrm{Y}^{-} \rightarrow \\
(\mathrm{M}-(n-3) \mathrm{H}+(n-1) \mathrm{X})^{+}+(n-3) \mathrm{HY} \quad(25)
\end{gathered}
$$

etc.

Figure 33 shows the post-ion/ion reaction spectrum that involved cations derived from PEG 4600 and used highmass anions $\left(\mathrm{F}^{-}\right.$transfer $)$and low mass anions $\left(\mathrm{H}^{+}\right.$transfer $)$ from PDCH. Clearly, two distributions are observed in the ion/ion reaction products. One results from $\mathrm{F}^{-}$transfer, and the other results from $\mathrm{H}^{+}$transfer. A spacing of $22 \mathrm{Da}$ between adjacent peaks is observed as a result of the proton transfer mechanism due to the difference between the mass of sodium and that of the proton. For example, the quadruply charged oligomer $(\mathrm{M}+4 \mathrm{Na})^{4+}$ yields a singly charged product of $(\mathrm{M}+4 \mathrm{Na}-3 \mathrm{H})^{+}$, whereas the triply charged oligomer yields a singly charged product of $(\mathrm{M}+3 \mathrm{Na}-2 \mathrm{H})^{+}$, which is a difference in mass of 22 Da.

For ions of the type $(\mathrm{M}+\mathrm{nNa})^{\mathrm{n}+}$, sodium ion transfer is the best reaction type for mixture analysis. This reaction was not observed with any of the limited set of anions used to study PEG cations. However, the behavior of $\mathrm{I}^{-}$in reactions with PEG cations suggests that there may be anions that react by $\mathrm{Na}^{+}$transfer. In direct analogy with cations derived from proteins, iodide anion was found to attach to multiply charged PEG ions. As already discussed in relation to reaction (20), anion attachment is undesirable for mixture analysis. However, collisional activation studies showed that the iodide/PEG attachment product is an intermediate to sodium cation transfer, and not an intermediate to proton transfer as with multiply protonated protein/iodide adducts. This observation implies that, if sufficient energy is imparted to the PEG/iodide adducts, then they can be disrupted so that the net effect is the desired transfer of sodium ions, leading to a distribution of $(\mathrm{M}+\mathrm{Na})^{+}$ions.

Figure 34 illustrates this possibility. Figure 34(a) shows the spectrum acquired after the initial charge states of PEG 2000 (mostly the +2 ions with a lesser abundance of +3 ions) were subjected to reactions with iodide anions. Two major distributions are apparent in the singly charged product ions. One is associated with single iodide attachment to the $(\mathrm{M}+2 \mathrm{Na})^{2+}$ precursor ions, and the other is associated with the attachment of two iodide anions to the $(\mathrm{M}+3 \mathrm{Na})^{3+}$ precursor ions. The spectrum of Fig. 34(b) was acquired after adding a collisional activation period following the ion/ion reaction period that produced the product ions of Fig. 34(a). This collisional activation period involved a sweep of the rf amplitude applied to the ring-electrode while applying a $75-\mathrm{kHz}$ sine-wave to the end-cap electrodes. The amplitude was selected to maximize the collisional dissociation of the singly charged adduct ions and to avoid ejecting them from the ion trap. The doubly charged ions are ejected at this amplitude. Note that the initial singly charged product ion distribution is largely converted to $\mathrm{M}+\mathrm{Na}^{+}$ions. There is a distribution of relatively low abundance $(\mathrm{M}+2 \mathrm{Na}+\mathrm{I})^{+}$ions that arises from either incomplete dissociation of the adducts that contain a single iodide, or, more likely, from a fraction of the $(\mathrm{M}+3 \mathrm{Na}+2 \mathrm{I})^{+}$ions that do not fragment by loss of two molecules of NaI (or a combination of both). However, this group of ions could probably be dissociated with a second sweep of collisional activation. In any case, this experiment shows the desirability of reducing the charge on PEG ions to the +1 charge-state via sodium ion transfer. Unfortunately, the technique demonstrated here for the PEG 2000 ions is decreasingly effective in the ion trap for larger PEGs, because ion ejection becomes increasingly competitive with collisional dissociation as the mass of the PEG ions increases. The behavior of $\mathrm{I}^{-}$shows that there are anions that prefer to react by sodium transfer rather than by proton transfer, and suggests that there may be a more appropriate choice of anion, specifically one that leads to a weaker binding interaction for the adduct (see discussion of $\mathrm{HI}$ attachment to proteins in Section IV.C).

\section{B. Formation of Precursor lons of Low Charge for MS/MS}

In addition to its use in complex mixture analysis, as described in the previous section, charge-state manipulation 

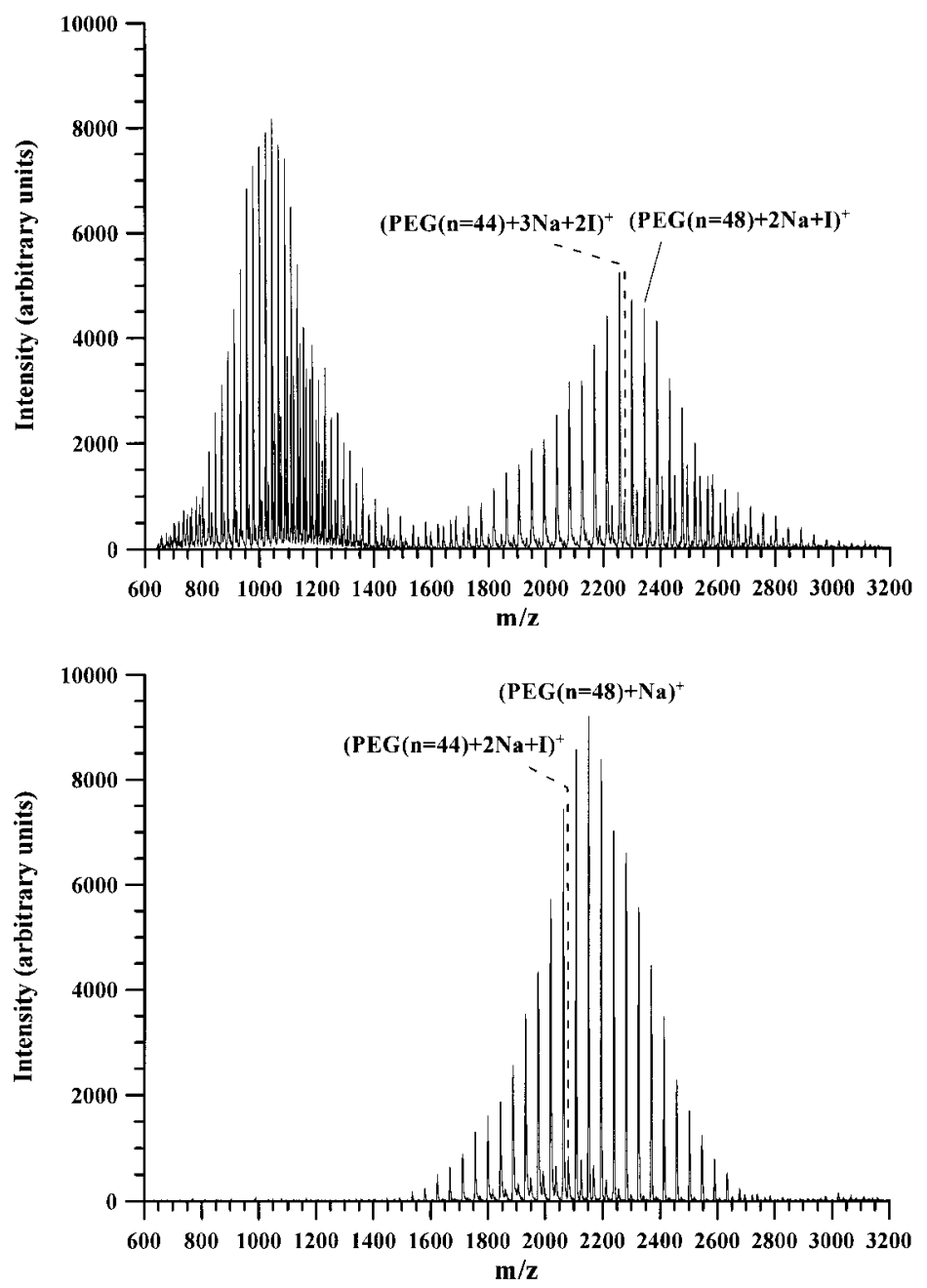

FIGURE 34. (a) Positive ion electropsray mass spectrum of PEG 2000 after a $196-\mathrm{ms}$ reaction period with $\mathrm{I}^{-}$. (b) Spectrum obtained after the ions of (a) were subjected to a short collisional excitation period. (Reprinted from the J Am Soc Mass Spectrom 1998, 9, 957-965, with permission from Elsevier Science, Inc. Copyright 1998 by the American Society for Mass Spectrometry.)

may also be desirable in some instances in which electrospray is applied to pure compounds. One such application is in the formation of precursor ions of lower charge than those produced directly by electrospray. The ions thus formed might be studied, for example, for their mobilities (Clemmer \& Jarrold, 1997), unimolecular chemistry, or bimolecular chemistry. It is well known that electrospray tends to yield a distribution of charge states, the composition of which tends to be dependent upon the molecule of interest and ionization/ interface conditions (Wang \& Cole, 1997). It is often difficult, by modification of ionization conditions alone, to produce a useful abundance of each and every charge state that extends from \pm 1 to the highest charge states achievable with electrospray. For this reason, it is desirable to have a means to reduce high ion charge states to lower charge states that are independent of ionization/interface conditions. As already developed in previous sections, ion/ion chemistry is particularly advantageous in this regard, especially in producing ions of very low charge from high charge-state ions. For the purpose of illustration, two examples are given here in which ion/ion chemistry was used to produce sufficient numbers of precursor ions from charge states not formed directly by electrospray. Two examples describe the production of precursor ions for (i) subsequent collisional activation and (ii) ion/molecule reaction chemistry studies.

Figure 35 shows four MS/MS spectra that are derived from the ion trap collisional activation of the $(\mathrm{M}+\mathrm{nH})^{\mathrm{n}+}$ ions of melittin, where $\mathrm{n}=1-4$. The electrospray mass spectrum for melittin was dominated by the $(\mathrm{M}+4 \mathrm{H})^{4+}$ ion with a much lower abundance of the $(\mathrm{M}+3 \mathrm{H})^{3+}$ ion. Essentially no doubly or singly protonated ions were formed directly via electrospray. A greater abundance of $(\mathrm{M}+3 \mathrm{H})^{3+}$ could be formed for subsequent MS/MS with a short reaction period (few tens of ms) with anions derived from PDCH. At 

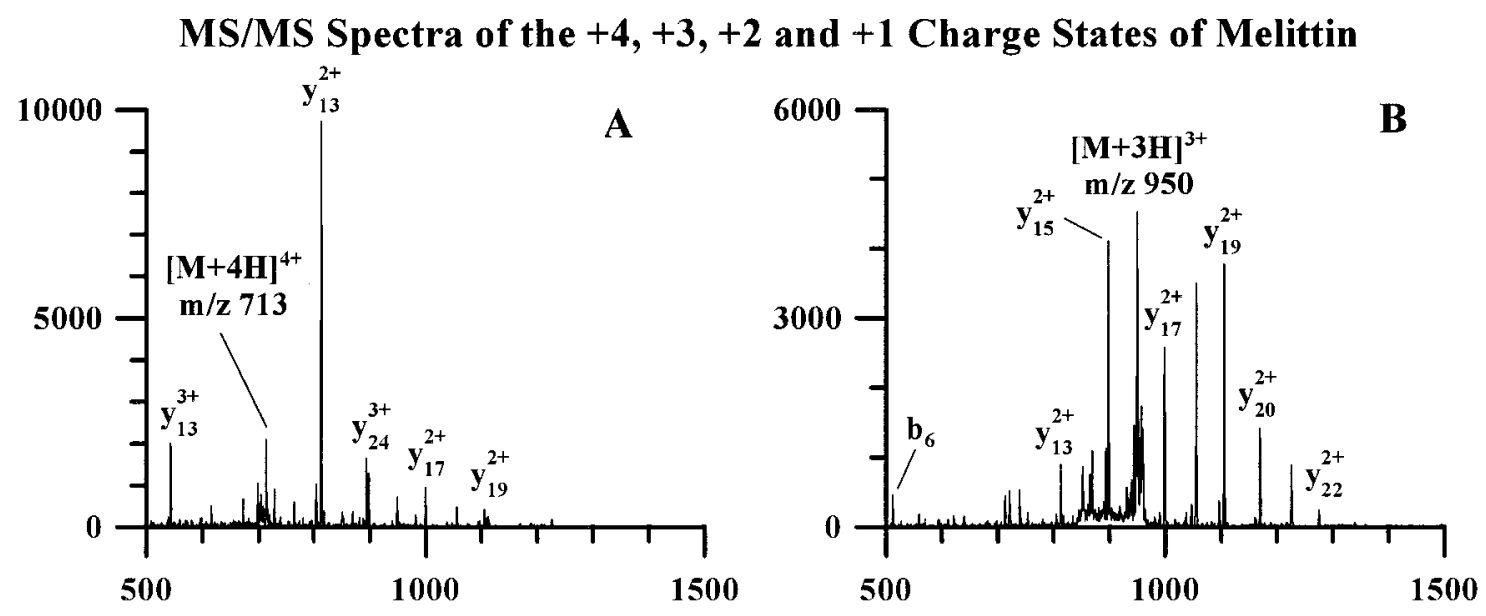

ge States of Melittin
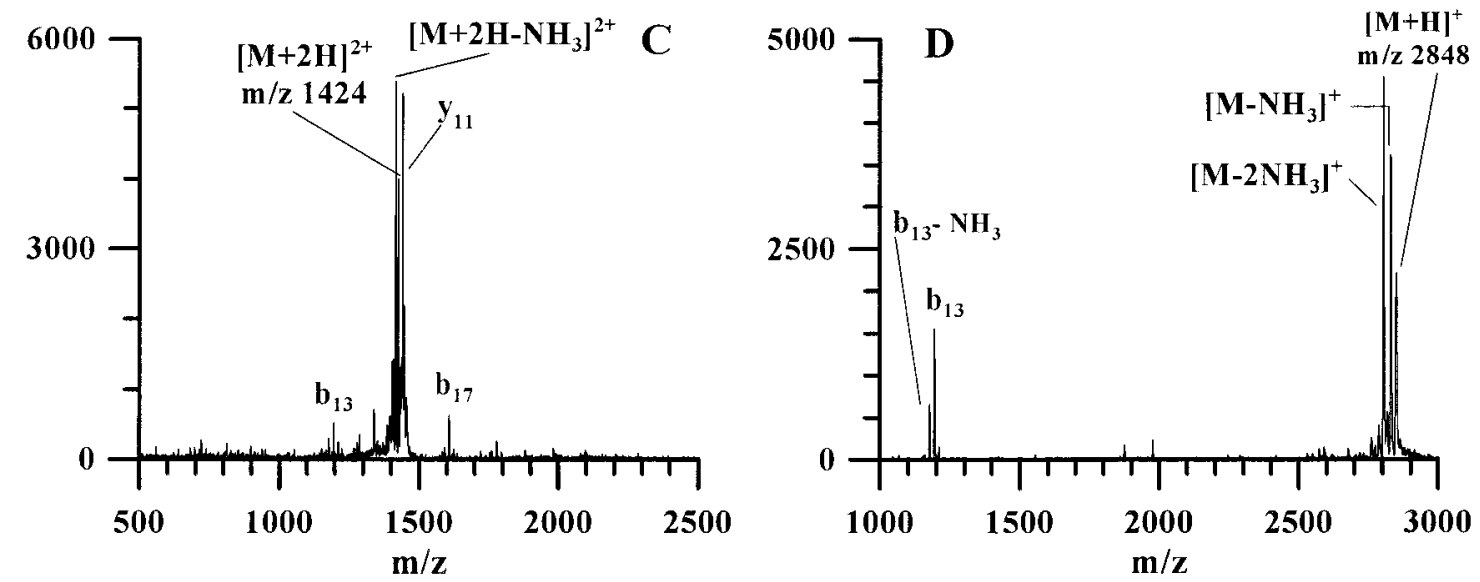

FIGURE 35. Ion trap MS/MS spectra derived from collisional activation of the (a) +4 , (b) +3 , (c) +2 , and (d) +1 charge states of melittin. Most of the $(\mathrm{M}+3 \mathrm{H})^{+}$and essentially all the $(\mathrm{M}+2 \mathrm{H})^{2+}$ and $(\mathrm{M}+\mathrm{H})^{+}$ions were formed via ion/ion proton transfer reactions that involved the $(\mathrm{M}+4 \mathrm{H})^{4+}$ ions.

increasingly longer reaction times, the doubly and singly protonated species could, in turn, be made the base peak in the spectrum. The formation of the singly protonated species is particularly noteworthy ,because, in a previous ion/molecule reaction study, no doubly charged ions derived from melittin (precursor or product) could be deprotonated by the strong gaseous base 1,6-hexanediamine (McLuckey et al., 1991a). Ion/ion chemistry, on the other hand, forms the singly protonated species from the higher charge states with the expected $Z^{2}$ rate dependence. It has commonly been observed for MS/MS spectra of biopolymers as a function of charge state that the identities and relative abundances of product ions can change dramatically from one charge state to the next. Melittin is no exception, as is apparent from the differences in the spectra of Fig. 35(a)-(d).

Figure 5(a) shows an electrospray mass spectrum of ubiquitin acquired under commonly used electrospray conditions. A range of charge states is observed, with the lowest being the +7 charge state. In a study of the ion/molecule chemistry of hydroiodic acid attachment to protein cations (Stephenson \& McLuckey, 1997e), the number of HI mole- cules that attached to the protein cation as a function of charge state was of interest. Under normal electrospray conditions, this study was, therefore, limited to the charge states +13 to +7 . Ion/ion reactions, however, could be used to generate sufficient numbers of +6 to +1 ions for study as well. Figure 36 gives an example of the ion/molecule reaction results for the +1 charge state. Anions derived from PDCH were used to reduce the multiply charged ubiquitin ions to the +1 charge state, and the +1 ions were stored in the presence of ca. $2 \times 1)^{-5}$ torr of HI for about $2 \mathrm{~s}$. By this time, the spectrum of products became essentially invariant. As can be seen in the figure, the distribution of clusters that resulted from this experiment reflects the attachment of 512 molecules of HI to the singly charged ion. This experiment, and those of all other charge states, supported the hypothesis that the sum of the maximum number of $\mathrm{HI}$ molecules to attach to the ion $\left[12\right.$ in the case of the $(\mathrm{M}+\mathrm{H})^{+}$ ion] and the charge [1 in the case of the $(\mathrm{M}+\mathrm{H})+$ ion] should equal the sum of the number of lysines, histidines, arginines, and $\mathrm{N}$-termini in the protein (13 in the case of ubiquitin). 


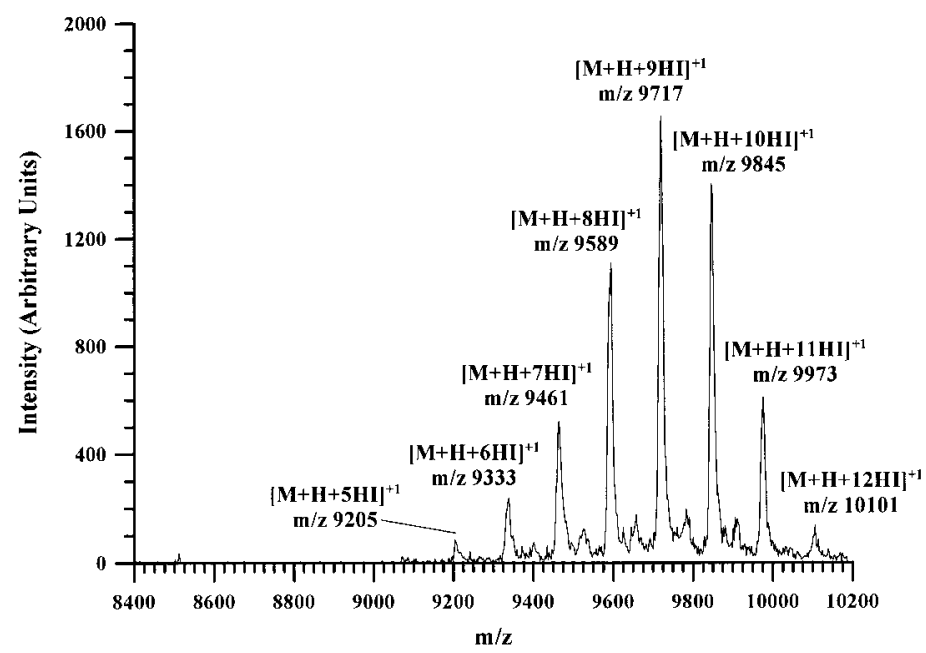

FIGURE 36. Positive ion mass spectrum obtained after +1 ions of bovine ubiquitin were formed via ion/ion proton transfer reactions from the +13 to +7 charge states and allowed to react with the hydroiodic acid admitted into the ion-trap vacuum system (roughly $2 \times 10^{-5}$ torr ) for $2 \mathrm{~s}$.

\section{Ion/Ion Reactions and Mass Determination}

Part A of this section emphasized the reduction of charge states to low values, principally +1 or -1 , within the context of complex mixture analysis. There are instances in which it may not be necessary, or desirable, to reduce charge states to such low values. In some cases, it may be preferable to retain a number of charge states to take advantage of multiple charging to make mass determinations via algorithms designed to yield "zero-charge"-spectra. Such algorithms are most likely to provide erroneous or ambiguous results when individual charge states are not base-line resolved and when chemical noise is high. Ion/ion chemistry can be used to provide better charge state resolution, by reducing charge states, and can lead to reduced relative levels of chemical noise. The latter effect has been attributed to a reversal of the normal tendency of electrospray to yield ions within a relatively narrow mass-to-charge window. That is, by reducing highly charged high-mass ions to lower charge states, ions of lower charge and lower mass that contribute to chemical noise in the normal electrospray mass spectrum no longer fall in the same mass-to-charge range as the higher mass species.

As analyte mass increases, the envelope of peaks for each charge state increases in width due to the increasingly broad isotopic distribution. Furthermore, in the case of proteins, the likelihood for microheterogeneity and glycosylation also tends to increase with biopolymer size. The absolute number of charges also tends to increase with mass, and leads to a decrease in the spacing between charge states. Therefore, high-mass analytes and mixtures of analytes can lead to poor peak definition. Compounding the situation further is the fact that most chemical noise also appears in the same electrospray mass-to-charge window. The resulting spectral congestion can, therefore, challenge the capability of an algorithm to yield a reliable zero-charge spectrum. Figures 37 and 38 illustrate the utility of partial charge state reduction for mass determination and for the identification of minor mixture components (Stephenson \& McLuckey, 1998b). Figure 37(a) shows the normal electrospray mass spectrum of chicken conalbumin, and Fig. 37(b) shows the transformed spectrum. The transformed spectrum shows as many as seven "peaks" at regular spacing. There is no obvious basis on which to select one of these peaks as that representing the molecular mass of the protein. The multiple peaks that appear in the transformed spectrum are analogous to the multiple candidate masses that can arise from the uncertainty in assigning charge states when making manual interpretations of spectra with closely spaced, poorly defined peaks.

Figure 38(a) shows the spectrum of conalbumin obtained after the ion population reflected in Fig. 37(a) was subjected to ion/ion proton transfer reactions with anions derived from PDCH for $100 \mathrm{~ms}$. Figure 38(a) shows a charge state range of +2 to +6 , in contrast to the range of +36 to +52 shown in Fig. 37(a). In the case of Fig. 38(a), charge states can easily be assigned with confidence with manual interpretation of the spectrum. Likewise, the transformed spectrum of Fig. 38(b) yields far less ambiguous information than that of Fig. 37(b). Also apparent in both spectra of Fig. 38 are minor components in the spectra that are not clearly apparent in the data of Fig. 37. For example, a peak on the low mass-to-charge side of each of the conalbumin ion signals is clearly observed for all charge states in Fig. 38(a), and the species that give rise to these ions is also reflected in the transformed spectrum. At least one other component that yields much lower ion abundance than either conalbumin or its low mass neighbor is also reflected in Fig. 38 (a), and a small transformed peak appears at roughly $66 \mathrm{kDa}$ 

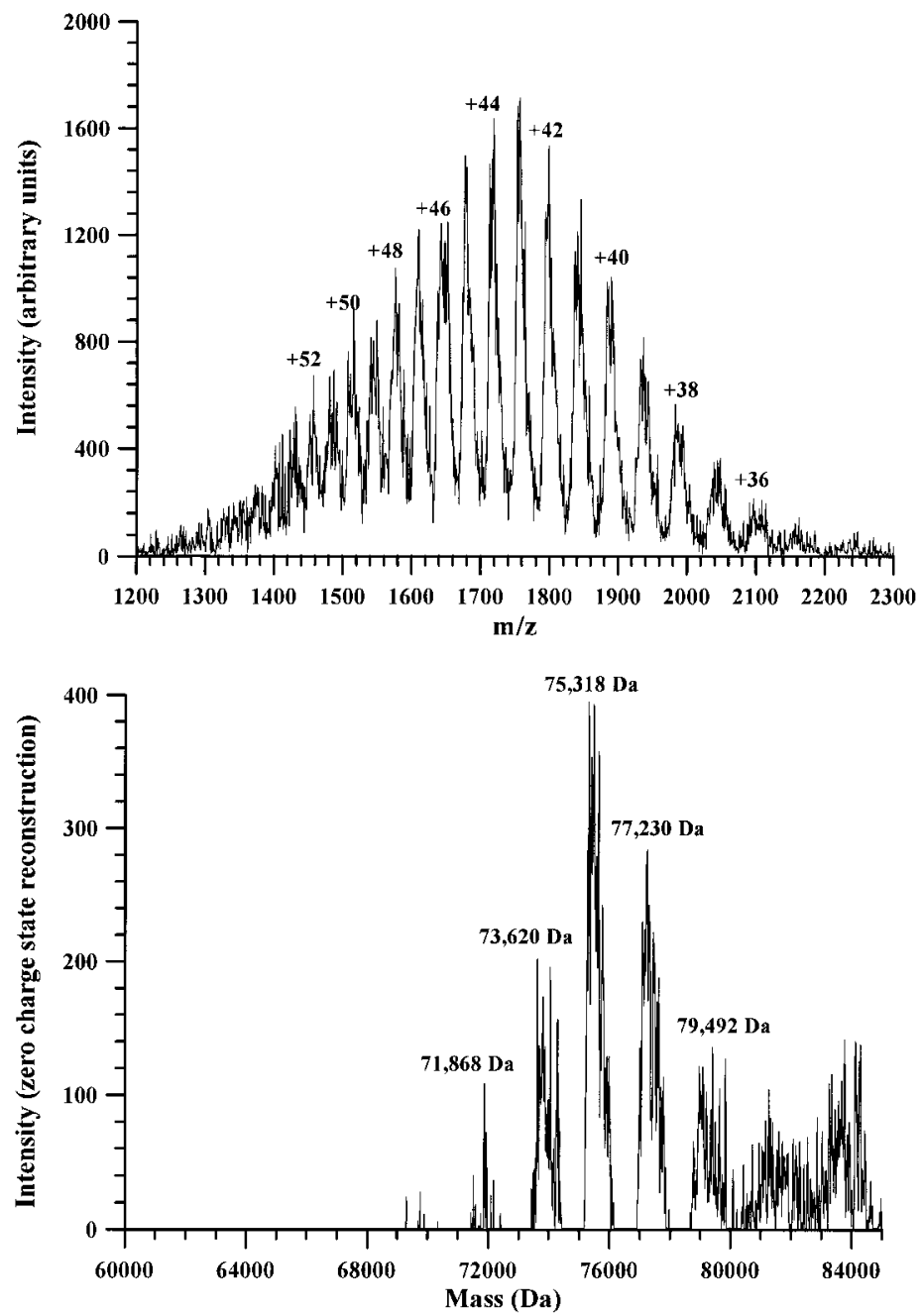

FIGURE 37. (a) Electrospray mass spectrum of chicken conalbumin and (b) the transformed spectrum. (Reprinted from J Mass Spectrom 1998, 33, 664-672 with permission. Copyright 1998 John Wiley \& Sons, Ltd.)

in Fig. 38(b) that corresponds to this component. Clearly, the data of Fig. 38 provide more reliable mass assignments than the data of Fig. 37, and also exhibit minor components that are present in the original ion population that are not apparent, but must be present, in the original electrospray mass spectrum. This improved performance can be attributed to the betterresolved and better-defined peaks after charge state manipulation, and the lower noise levels in the data acquired after the ion/ion reactions.

\section{Interpretation of Product-Ion Spectra}

The determination of ion charge is an issue common to most electrospray mass spectrometry experiments that involve high-mass species. For mass spectra of high-mass species that show a series of coherent charge states, the determination of charge is straightforward and involves the solution of a set of simultaneous equations (Mann et al., 1989). MS/MS spectra of multiply charged precursor ions, on the other hand, can be more problematic, because there is no defined relationship between adjacent peaks. When two charged products from the same dissociation (referred to as complementary ions) appear in the MS/MS spectrum, they can often be identified based on the ratio of $\mathrm{m} / \mathrm{z}$ spacings between the product ions and the precursor ion. However, both complementary ions are often not observed. Measurement of the spacings of the isotope peaks is a definitive way to determine charge states (Kent \& McLafferty, 1990), and this approach is often used to determine product-ion charge states. The ability to determine charge states for high-mass/ high-charge fragments, however, is ultimately limited by the resolving power of the mass analyzer, and is, therefore, most effectively accomplished with Fourier-transform ion cyclotron resonance mass spectrometry. 

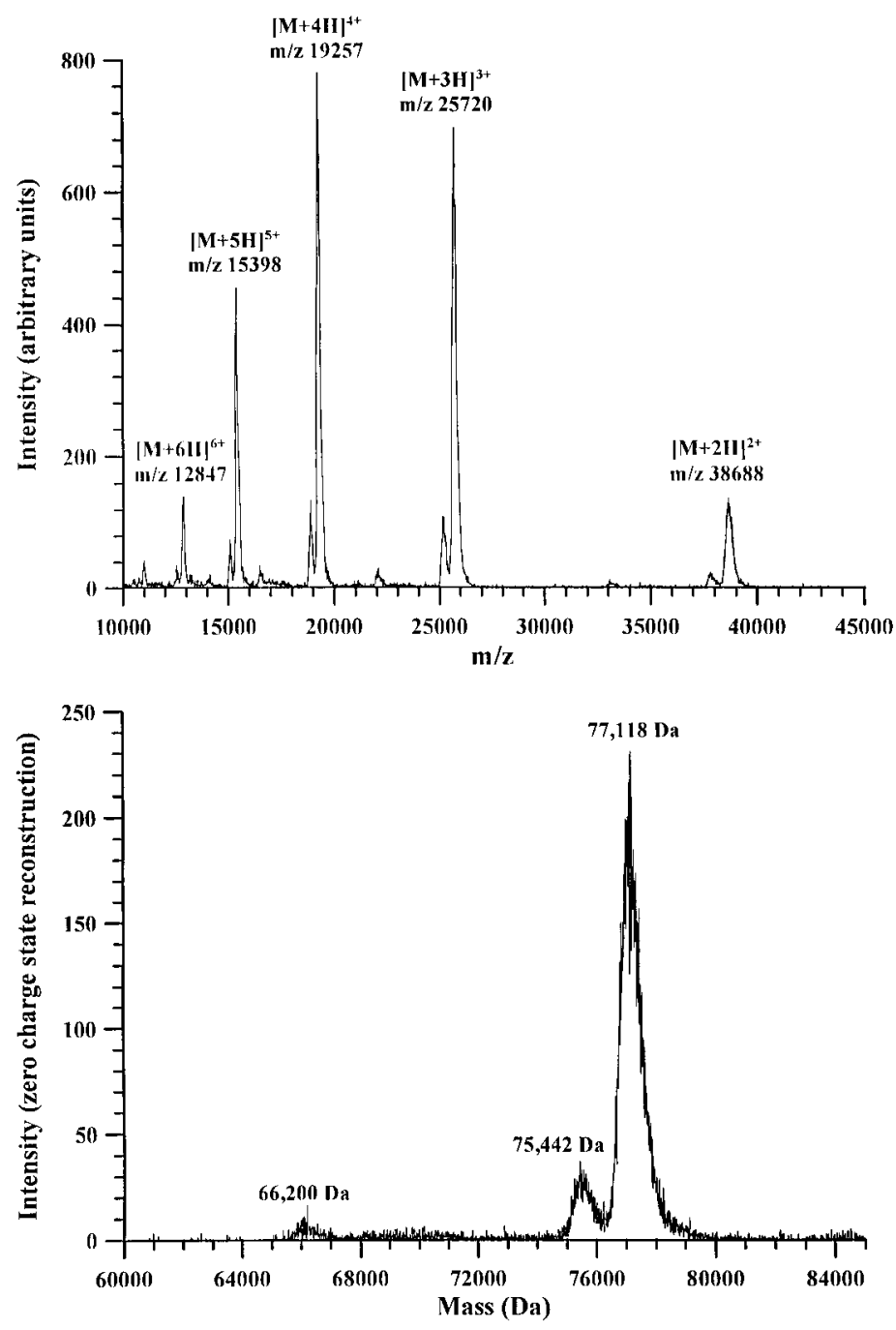

FIGURE 38. Positive ion electrospray mass spectrum of chicken conalbumin obtained after the ion population of Fig. 37(a) was subjected to ion/ion proton transfer reactions with $\mathrm{PDCH}$ anions for $100 \mathrm{~ms}$ and (b) the transformed spectrum. (Reprinted from J Mass Spectrom 1998, 33, 664-672 with permission. Copyright 1998 John Wiley \& Sons, Ltd.)

Product-ion charge states can also be determined via charge transfer reactions. Product-ion charge state determination via ion chemistry was first demonstrated with proton transfer ion/molecule chemistry with multiply charged polypeptides (McLuckey et al., 1991a; Hunter et al., 1994). In this approach, proton transfer reactions are used to provide at least two charge states from a product ion so that ion charges can be established in the same way that they usually are with electrospray mass spectra. Unfortunately, ion/molecule proton transfer chemistry proved not to be universal, and clustering reactions sometimes compete with proton transfer (McLuckey et al., 1991a). As already demonstrated earlier in this review, ion/ion chemistry is "universal," and with the proper selection of reactant ions, proton transfer can be the exclusive reaction type. The use of ion/ion reactions for product-ion charge-state determination has been de- scribed for multiply charged negative ions in reactions with protonated pyridine (Herron, et al., 1996b). Figure 39 shows, for example, the results of an $\mathrm{MS}^{3}$ experiment whereby two product ions from the $(\mathrm{M}-6 \mathrm{H})^{6-}$ precursor ion from oxidized bovine insulin A-chain that fell within a mass-tocharge window of 475-505 were subjected to ion/ion proton transfer reactions with protonated pyridine. The peak spacings indicate that the most abundant of the two product ions was a -5 ion, and that the ion of lower abundance was a -4 ion. With the charge being established, it was possible to assign these ions as being the $\mathrm{C}_{20}$ and $\mathrm{Z}_{16}$ products, respectively. By use of a series of $\mathrm{MS}^{3}$ experiments, it was possible to assign the charge state of every major product ion in the MS/MS spectrum.

Armed with a more thorough understanding of ion/ion reaction kinetics, it became apparent that another approach 


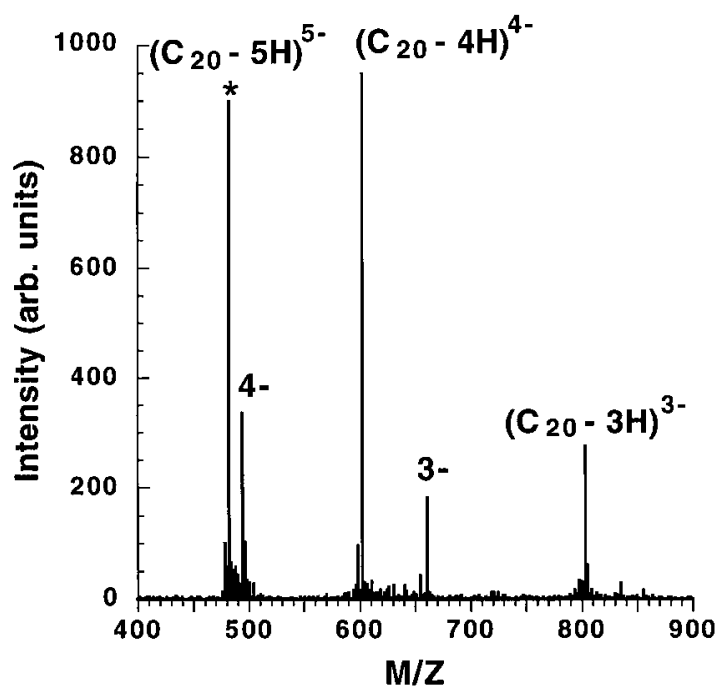

FIGURE 39. Product-ion spectrum that arises from the $\mathrm{MS}^{3}$ experiment in which the collisional activation product ions from $(\mathrm{M}-6 \mathrm{H})^{6-}$ of oxidized bovine insulin A-chain within the $\mathrm{m} / z$ range of $475-505$ were subjected to ion/ion proton transfer reactions with PDCH anions. (Reprinted with permission from Anal Chem1996, 68, 257-262, copyright 1996 American Chemical Society.)

to simplify the interpretation of product ion spectra derived from multiply charged precursor ions would be to convert each product ion to a singly charged ion by ion/ion chemistry (Stephenson \& McLuckey, 1998c). A product ion spectrum is simply a mixture of ions of varying mass and charge [similar to the electrospray mass spectrum of a mixture of components (see Section V.A)], and is therefore amenable to conversion to a largely singly charged product ion spectrum without complete loss of any of the product ions due to total neutralization. Conversion of the entire set of product ions to singly charged ions obviates the use of a series of $\mathrm{MS}^{3}$ experiments to determine product ion charge states. Figure 40 provides a comparison of pre- and post-ion/ion reaction data for the product ions generated by ion-trap collisional activation from the $(\mathrm{M}+8 \mathrm{H})^{8+}$ precursor ions of ubiquitin. The ion/ion reactions were allowed to proceed for times long enough (ca. $120 \mathrm{~ms}$ ) to ensure that all ions were driven primarily to the +1 charge state. Residual doubly charged ions can be readily identified by their positions on the mass scale. Under these conditions, a much more abundant singly charged ion of twice the mass-to-charge (less the mass of a proton) must appear in the spectrum for a peak to be ascribed to a doubly charged ion. This comparison, and a variety of others, demonstrated that the post-ion/ion reaction product ion spectra were more readily interpreted, because there were far fewer ambiguities on the assignment of charge.

Perhaps a more dramatic demonstration of the use of ion/ion reactions to simplify product ion spectra is shown in the comparison of the spectra shown in Fig. 41(a) and 41(b). In this case, product ions were formed by creating a voltage gradient in the atmosphere/vacuum interface sufficient to induce dissociation (no precursor ion isolation). The spectrum derived from tuna cytochrome $c$ with such interface conditions is shown in Fig. 41(a), with the intensity scale multiplied by a factor of 6.25 to highlight the ill-defined signals between the charge states. These signals appear as a result of the use of relatively violent interface conditions, and are due primarily to fragmentation of the higher charge states of cytochrome $c$. The product ions thus produced constitute a mixture of ions far too complex for the ion-trap mass analyzer. Figure 41(b), on the other hand, shows a spectrum of the same ion population after ion/ion proton transfer reactions drive the charges predominantly to +1 . A large majority of product ions could be assigned as either y-type or b-type ions. The comparison of Fig. 41 clearly shows that more primary sequence information can be obtained from interface-induced dissociation of a small-tomoderate sized protein with an ion-trap mass spectrometer when ion/ion reactions are used to reduce product ion charges to +1 .

\section{CONCLUSIONS}

Multiply charged ion/ion chemistry has been studied at near-atmospheric pressure in a reaction region that leads to the atmospheric/vacuum interface of a mass spectrometer, and within a quadrupole ion trap operated with a bath gas at a pressure of 1 mtorr. Even for the relatively limited set of positive ion/negative ion reactant combinations thus far investigated, a wide range of reaction phenomenology has been noted. Proton transfer has been the most common reaction type for high-mass ions derived from electrospray, but other forms of "charge transfer," such as electron transfer and fluoride transfer, have also been observed. For some ion/ion reactions, the attachment of the two reactants has been observed. For the latter cases, a mechanism for cooling the ion/ion collision complex that is fast enough to compete with its dissociation is required. For the techniques used to study multiply charged ion/ion chemistry to date, this role has been fulfilled by collisions with a background gas. The reaction type that is observed is determined by the chemical nature of the reactants. For a given singly charged reagent ion, multiply charged ions of opposite polarity tend to react via the same major mechanism within a compound class. For example, all positively charged polypeptides studied thus far react with even-electron perfluorocarbon anions by proton transfer.

In considering the kinetics and thermodynamics of the reactions, it is apparent that multiply charged ion/ion reactions are relatively fast, due to the long-range Coulombic attraction. They are also universal,because any pair of oppositely charged ions is expected to react in some way due to the high exothermicity that is associated with mutual neu- 

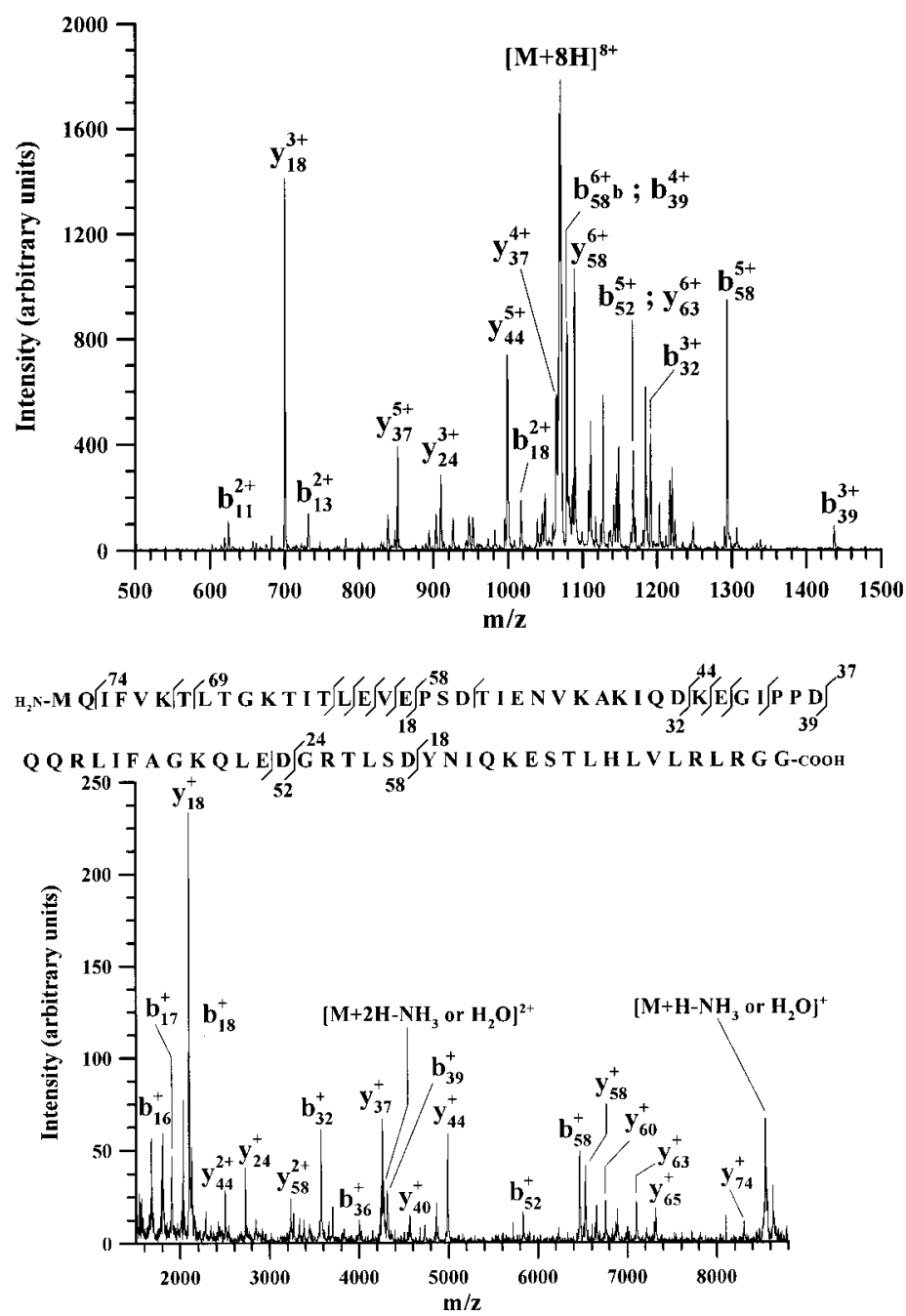

FIGURE 40. (a) Pre-ion/ion reaction product ion spectrum derived from ion-trap collisional activation of the $(\mathrm{M}+8 \mathrm{H})^{8+}$ ion of bovine ubiquitin and (b) the post-ion/ion reaction product-ion spectrum. (Reprinted with permission from Anal Chem 1998, 70, 3533-3544, copyright 1998 American Chemical Society.)

tralization. The kinetics of reaction for multiply charged ions derived from the same molecule with a given singly charged reactant ion follow a $\mathrm{Z}^{2}$ dependence, at least under usual quadrupole ion-trap conditions. This dependence suggests that reaction rates are determined by the long-range Coulomb attraction, and that the ions react with constant efficiency as a function of charge state. As a result of the high reaction exothermicities, ion/ion reaction kinetics are not expected to be sensitive to the three-dimensional structures of the ions.

The degree to which products of ion/ion reactions dissociate as a result of the high exothermicity associated with the reaction depends upon the magnitude of the exothermicity, the partitioning of the reaction energy between all available states, the kinetic stability of the product ions, production size, and the rate at which excess product-ion internal energy is removed by collisions and emission. In the case of proton transfer reactions from polypeptides to even-electron perfluorocarbon anions, no fragmentation of the polypeptide product ions has as yet been observed. Electron transfer from small oligonucleotide anions to rare gas cations, on the other hand, results in extensive fragmentation of the nucleic acid product ions. The extent of fragmentation decreases as the size of the oligonucleotide anions increases; that relationship reflects an increase in ion lifetimes. When ion-cooling rates become competitive with dissociation rates, the initially formed product ions are stabilized, and fragmentation is avoided. Reaction models suggest that a significant degree of collisional cooling in the quadrupole ion trap will be experienced by product ions formed with dissociation rates less than about $10^{4} \mathrm{~s}^{-1}$. Collisional cooling, therefore, likely plays an important role in the relative lack of dissociation observed for most high-mass ions in the ion trap as a result of ion/ion reactions. 

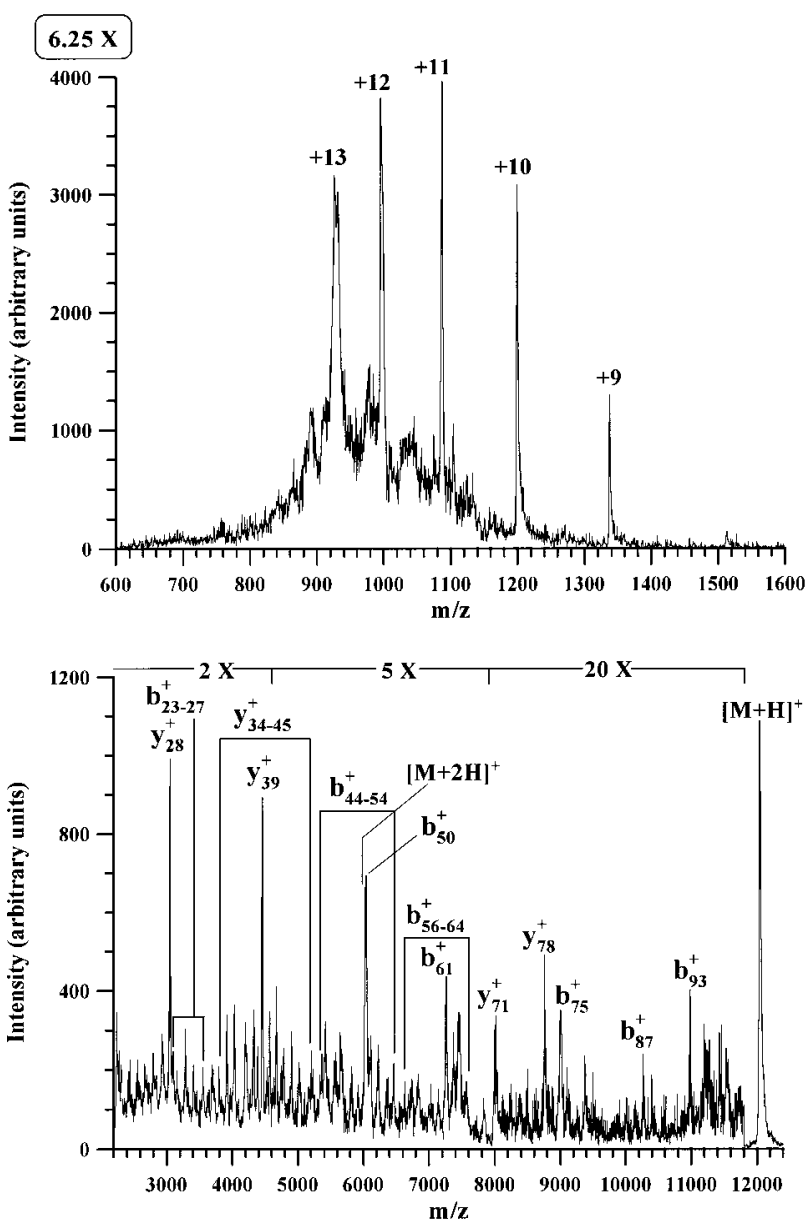

FIGURE 41. (a) Positive ion electrospray mass spectrum of tuna cytochrome $\mathrm{c}$ obtained under interface conditions chosen to induce fragmentation, and (b) the spectrum obtained after the ions of (a) were subjected to ion/ion proton transfer reactions with anions from PDCH. (Reprinted with permission from Anal Chem 1998, 70, 3533-3544, copyright 1998 American Chemical Society.)

The observed dependence of ion/ion reaction rates on the square of the ion charge, the universal nature of mutual neutralization (arising from the high exothermicities of the reactions), and the relative lack of fragmentation from ion/ ion reactions, at least for high-mass ions stored in the presence of a light bath gas at 1 mtorr, makes ion/ion chemistry a particularly useful means to manipulate charge states. Application to mixtures of like species (e.g., proteins, oligonucleotides, etc.) that yield ions of a wide range of masses and charges when subjected to electrospray can greatly reduce the complexity of interpreting mass spectra. Ion/ion chemistry can be used to reduce charge states to mostly +1 or -1 without completely neutralizing any of the mixture components, or ion/ion chemistry can be used simply to reduce charge states to the point where they are readily resolved so that algorithms intended to yield "zero-charge" spectra are more effective. Ion/ion reactions are particularly useful to form low-charge state precursor ions from more highly charged precursor ions for subsequent MS/MS or $\mathrm{MS}^{n}$ experiments. Ion/ion chemistry can also be used to simplify the interpretation of product ion spectra derived from multiply charged precursor ions. One strategy might involve a series of $\mathrm{MS}^{3}$ experiments, whereby the charge state of the various product ions could be determined unambiguously. Another strategy might involve the conversion of the product-ion spectrum largely to a spectrum of singly charged products.

The major potential application areas for gas-phase ion/ ion chemistry described here involve proton transfer. Based on the interesting, but still relatively small, number of observations thus far made for multiply charged ion/ion gasphase reactions, it is clear that the chemistry associated with them can be far richer than proton transfer. Multiply charged ion/ion reactions have thus far been studied over a very limited set of experimental conditions. Other instrumental approaches to study these reactions will likely lead to new insights and to new phenomena. Furthermore, the range of possible positive ion/negative ion combinations is still largely unexplored. In fact, the variety of multiply charged ion/ion reactant combinations that can be readily studied is potentially far greater than the range of multiply charged ion/molecule reactant combinations. The neutral reactants for gas-phase ion/molecule chemistry are largely restricted to relatively small volatile species, whereas the mass spectrometry community has developed a variety of means for forming a dazzling array of gaseous ions. For this reason, it seems likely that many new reaction phenomenologies will be observed, some of which can lead to novel or improved mass spectrometry applications.

Note added in proof: The reader is directed to an ion/ion chemistry paper that appeared after acceptance of this review. The citation is "Scalf, M.; Westphall, M. S.; Krause, J.; Kaufman, S. L.; Smith, L. M. Science, 1999; 283, $194-$ 197.',

\section{REFERENCES}

1. Axelsson, J.; Reimann, C. T.; Sundqvist, B. U. R. Int J Mass Spectrom Ion Proc 1994, 133, 141-155.

2. Baranov, V.; Bohme, D. E. Int J Mass Spectrom Ion Proc 1997, 165/166, 249- 255.

3. Bates, D. R. In: Advances in Atomic and Molecular Physics, Vol. 20; Bates, D. R.; Bederson, B. (Eds.); Academic Press: Orlando, 1985; pp. 1-37.

4. Berberich, D. W.; Yost, R. A. J Am Soc Mass Spectrom 1994, 5, 757-764.

5. Brauman, J. I. J Mass Spectrom 1995, 30, 1649-1651.

6. Cassady, C. J.; Carr, S. R. J Mass Spectrom 1996, 31, 247254.

7. Cassady, C. J.; Wronka, J.; Kruppa, G. H.; Laukien, F. H. Rapid Commun Mass Spectrom 1994, 8, 394-400. 
8. Chen, Y.-L.; Campbell, J. M.; Collings, B. A.; Konermann, L.; Douglas, D. J. Rapid Commun Mass Spectrom1998, 12, 1003-1010.

9. Chen, Y.-L.; Collings, B. A.; Douglas, D. J. J Am Soc Mass Spectrom 1997, 8, 681-687.

10. Clemmer, D. E.; Jarrold, M. F. J Mass Spectrom 1997, 32, 577-592.

11. Cole, R. B., (Ed.); Electrospray Ionization Mass Spectrometry, Fundamentals, Instrumentation, and Applications; Wiley: New York, 1997.

12. Covey, T. R.; Douglas, D. J. J Am Soc Mass Spectrom 1993, 4, 616-623.

13. Creaser, C. S. In: Practical Aspects of Ion Trap Mass Spectrometry, Volume III; March, R. E.; Todd, J. F. J. (Eds.); CRC Press: Boca Raton, 1995; pp. 239-253.

14. Eckenrode, B. A.; Glish, G. L. ; McLuckey, S. A. Int J Mass Spectrom Ion Proc 1990, 99, 151-167.

15. Fenn, J. B.; Mann, M.; Meng, C. K.; Wong, S. F.; Whitehouse, C. M. Science 1990, 246, 64-71.

16. Ferrige, A. G.; Seddon, M. J.; Jarvis, S. Rapid Commun Mass Spectrom 1991, 5, 374-379.

17. Flannery, M. R. In: Applied Atomic Collision Physics, Vol. 3; McDaniel, E. W.; Nighan, W. L. (Eds.); Academic: New York, 1982a; pp. 141-172.

18. Flannery, M. R. Phil Trans R Soc Lond A 1982b, 304, $447-$ 497.

19. Gaskell, S. J. J Mass Spectrom 1997, 32, 677-688.

20. Goeringer, D. E.; McLuckey, S. A. Int J Mass Spectrom 1998, 177, 163-174.

21. Green, M. K.; Lebrilla, C. B. Mass Spectrom Rev. 1997, 16, 53-71.

22. Griffin, L. L.; McAdoo, D. J. J Am Soc Mass Spectrom 1993 , 4, 11-15.

23. Gross, D. S.; Zhao, Y. X.; Williams, E. R. J Am Soc Mass Spectrom 1997, 8, 519-524.

24. Herron, W. J.; Goeringer, D. E.; McLuckey, S. A. J Am Soc Mass Spectrom 1995a, 6, 529-532.

25. Herron, W. J.; Goeringer, D. E. ; McLuckey, S. A. J Am Chem Soc 1995b, 117, 11555-11562.

26. Herron, W. J.; Goeringer, D. E.; McLuckey, S. A. Rapid Commun Mass Spectrom 1996a, 10, 277-281.

27. Herron, W. J.; Goeringer, D. E. ; McLuckey, S. A. Anal Chem1996b, 68, 257-262.

28. Hunter, A. P.; Severs, J. C.; Harris, F. M.; Games, D. E. Rapid Commun Mass Spectrom 1994, 8, 417-422.

29. Ikonomou, M. G.; Kebarle, P. Int J Mass Spectrom Ion Proc 1992, 117, 283-298.

30. Johnson, J. V.; Yost, R. A.; Kelley, P. E.; Bradford, D. C. Anal Chem 1990, 62, 2162-2172.

31. Kaiser, R. E., Jr.; Cooks, R. G.; Stafford, G. C., Jr.; Syka, J. E. P.; Hemberger, P. H. Int J Mass Spectrom Ion Proc 1991, 106, 79-115.
32. Kaltashov, I. A.; Fenselau, C. C. J Am Chem Soc 1995, 117, 9906-9910.

33. Labowsky, M.; Whitehouse, C.; Fenn, J. B. Rapid Commun Mass Spectrom 1993, 7, 71-84.

34. Lifshitz, C.; Gibson, D.; Levsen, K.; Dotan, I. Int J Mass Spectrom Ion Proc 1981, 40, 157-165.

35. Limbach, P. A. Mass Spectrom Rev 1996, 15, 297-336.

36. Little, D. P.; Speir, J. P.; Senko, M. W.; O’Connor, P. B.; McLafferty, F. W. Anal Chem 1994, 66, 2809-2815.

37. Loo, J. A.; Pesch, R. Anal Chem 1994, 66, 3659-3663.

38. Louris, J. N.; Brodbelt-Lustig, J. S.; Cooks, R. G.; Glish, G. L.; Van Berkel, G. J.; McLuckey, S. A. Int J Mass Spectrom Ion Proc 1990, 96, 117-137.

39. Mahan, B. H. In: Advances in Chemical Physics, Vol. 23; Prigogine, I.; Rice, S. A. (Eds.); Wiley: New York, 1973;140.

40. Malek, R.; Wanczek, K. P. Int J Mass Spectrom Ion Proc 1996, 158, 199-214.

41. Mann, M; Meng, C. K.; Fenn, J. B. Anal Chem 1989, 61, 1702-1708

42. Mather, R. E.; Todd, J. F. J. Int J Mass Spectrom Ion Phys.1980, 33, 159-165.

43. McLafferty, F. W.; Guan, Z. Q.; Haupts, U.; Wood, T. D.; Kelleher, N. L. J Am Chem Soc 1998, 120, 4732-4740.

44. McLuckey, S. A.; Stephenson, Jr., J. L.; Asano, K. G. Anal Chem 1998, 70, 1198-1202.

45. McLuckey, S. A.; Vaidyanathan, G. Int J Mass Spectrom Ion Proc 1997a, 162, 1-16.

46. McLuckey, S. A.; Stephenson, Jr., J. L.; O’Hair, R. A. J. J Am Soc Mass Spectrom 1997b, 8, 148-154.

47. McLuckey, S. A.; Herron, W. J.; Stephenson, Jr., J. L.; Goeringer, D. E. J Mass Spectrom 1996a, 31, 1093-1100.

48. McLuckey, S. A.; Goeringer, D. E.; Asano, K. G.; Vaidyanathan, G.; Stephenson, Jr., J. L. Rapid Commun Mass Spectrom 1996b, 10, 287-298.

49. McLuckey, S. A.; Goeringer, D. E. Anal Chem 1995a, 67, 2493-2497.

50. McLuckey, S. A.; Van Berkel, G. J.; Glish, G. L.; Schwartz, J. C. In: Practical Aspects of Ion Trap Mass Spectrometry, Volume II; March, R. E.; Todd, J. F. J. (Eds.); CRC Press: Boca Raton, 1995b; 89-141.

51. McLuckey, S. A.; Ramsey, R. S. J Am Soc Mass Spectrom 1994, 5, 324-327.

52. McLuckey, S. A.; Glish, G. L.; Van Berkel, G. J Anal Chem 1991a, 63, 1971-1978.

53. McLuckey, S. A.; Glish, G. L.; Van Berkel, G. J. Int J Mass Spectrom Ion Proc 1991b, 106, 213-235.

54. McLuckey, S. A.; Van Berkel, G. J.; Glish, G. L. J Am Chem Soc 1990, 112, 5668-5670.

55. McLuckey, S. A.; Glish, G. L.; Asano, K. G.; Grant, B. C. Anal Chem 1988, 60, 2220-2228.

56. Montaudo, G. Trends Polym Sci 1996, 4, 81-86. 
57. Ni, J.; Mathews, M. A. A.; McCloskey, J. A. Rapid Commun Mass Spectrom 1997, 11, 535-540.

58. Nordhoff, E.; Kirpekar, F.; Roepstorff, P. Mass Spectrom Rev. 1996, 15, 67-138.

59. O’Connor P. B.; McLafferty, F. W. J. Am Chem Soc 1995, $117,12826-12831$.

60. O’Hair, R. A. J.; McLuckey, S. A. Int J Mass Spectrom Ion Proc 1997, 162, 183-202.

61. Ogorzalek-Loo, R. R.; Smith, R. D. J Mass Spectrom 1995, 30, 339-347.

62. Ogorzalek-Loo, R. R.; Loo, J. A.; Udseth, H. R.; Fulton, J. L.; Smith, R. D. Rapid Commun Mass Spectrom 1992a, 6, $159-165$

63. Ogozalek-Loo, R. R.; Udseth, H. R.; Smith, R. D. J Amer Soc Mass Spectrom 1992b, 3, 695-705.

64. Ogorzalek-Loo, R. R.; Usdeth, H. R.; Smith, R. D. J Phys Chem 1991, 95, 6412-6415.

65. Petrie, S.; Javahery, G.; Wincel, H.; Bohme, D. K. J Am Chem Soc 1993, 115, 6290-6294.

66. Price, W. D.; Schnier, P. D.; Williams, E. R. Anal Chem 1996, 68, 859-866

67. Ramsey, R. S.; McLuckey, S. A. J Microcol Sep 1997, 9, 523-528.

68. Roth, L. M.; Freiser, B. S. Mass Spectrom Rev. 1991, 10, 303-328.

69. Rutherford, E. Phil Mag 1897, 44, 422-440.

70. Saf, R.; Mirtl, C.; Hummel, K. Acta Polymer 1997, 48 513-526.

71. Sannes-Lowery, K. A.; Mack, D. P.; Hu, P.; Loo, J. A. J Am Soc Mass Spectrom 1997, 8, 90-95.

72. Scheller, M. K.; Compton, R. N.; Cederbaum, L. S. Science 1995, 270, 1160-1166.

73. Schnier, P. D.; Gross, D. S.; Williams, E. R. J Am Chem Soc 1995, 117, 6747-6757.

74. Senko, M. W.; Speir, J. P.; McLafferty, F. W. Anal Chem 1994, 66, 2801-2808

75. Smith, D.; Adams, N. G.; Church, M. J. Planet Space Sci 1976, 24, 697-703.

76. Smith, R. D.; Cheng, X.; Bruce, J. E.; Hofstadler, S. A.; Anderson, G. A. Nature 1994, 369, 137-139.

77. Smith, R. D.; Loo, J. A.; Edmonds, C. G.; Barinaga, C. J.; Udseth, H. R. Anal Chem 1990a, 62, 882-899.

78. Smith, R. D.; Loo, J. A.; Barinaga, C. J.; Edmonds, C. G.; Udseth, H. R. J Am Soc Mass Spectrom 1990b, 1, 53-65.

79. Stephenson, Jr., J. L.; McLuckey, S. A. J Am Soc Mass Spectrom 1998a, 9, 585-596.

80. Stephenson, Jr., J. L.; McLuckey, S. A. J Mass Spectrom 1998b, 33, 664-672.

81. Stephenson, Jr., J. L.; McLuckey, S. A. Anal Chem 1998c, 70, 3533-3544.

82. Stephenson, Jr., J. L.; McLuckey, S. A. J Am Soc Mass Spectrom 1998d, 9, 957-965.
83. Stephenson, Jr., J. L.; McLuckey, S. A. Int J Mass Spectrom Ion Proc 1997a, 162, 89-106.

84. Stephenson, Jr., J. L.; Van Berkel, G. J.; McLuckey, S. A. J Am Soc Mass Spectrom 1997b, 8, 637-644.

85. Stephenson, Jr., J. L.; McLuckey, S. A. Int J Mass Spectrom Ion Proc 1997c, 165/166, 419-431.

86. Stephenson, Jr., J. L.; McLuckey, S. A. Anal Chem 1997d, $69,3760-3766$.

87. Stephenson, Jr., J. L.; McLuckey, S. A. J Am Chem Soc 1997e, 119, 1688-1696.

88. Stephenson, Jr., J. L.; McLuckey, S. A. Anal Chem 1997f, 69 , 281-285.

89. Stephenson, Jr., J. L.; McLuckey, S. A. Rapid Commun Mass Spectrom 1997g, 11, 875-880.

90. Stephenson, Jr., J. L.; McLuckey, S. A. J Am Chem Soc 1996a, 118, 7390-7397.

91. Stephenson, Jr., J. L.; McLuckey, S. A. Anal Chem 1996b, $68,4026-4032$

92. Suckau, D.; Shi, Y.; Beu, S. C.; Senko, M. W.; Quinn, J. P.; Wampler, F. M. III; McLafferty, F. W. Proc Natl Acad Sci USA 1993, 90, 790-793.

93. Tang, X.-J.; Boyd, R. K. Rapid Commun Mass Spectrom $1992,6,651-657$

94. Tardy, D. C.; Rabinovitch, B. S. Chem Rev 1977, 11, 369 408 .

95. Thomson, J. J.; Rutherford, E. Phil Mag 1896, 42, 392-407.

96. Tonkyn, R.; Weisshaar, J. C. J Am Chem Soc 1986, 108, $7128-7130$.

97. Vékey, K. Mass Spectrom Rev 1995, 14, 195-225.

98. Wang, G.; Cole, R. B. In: Electrospray Ionization Mass Spectrometry; Cole, R. B. (Ed.); Wiley-Interscience: New York, 1997; pp. 138-174.

99. Wang, P.; Bartlett, M. G.; Martin, L. B. Rapid Commun Mass Spectrom 1997, 11, 846-856.

100. Williams, E. R. J Mass Spectrom 1996, 31, 831-842.

101. Williams, J. D.; Cooks, R. G. Rapid Commun Mass Spectrom 1993, 7, 380-382.

102. Winger, B. E.; Light-Wahl, K. J.; Rockwood, A. L.; Smith, R. D. J Am Chem Soc 1992, 114, 5897-5898.

103. Wong, S. F.; Meng, C. K.; Fenn, J. B. J Phys Chem 1988, 92, $546-550$

104. Wood, T. D.; Chorush, R. A.; Wampler, F. M. III; Little, D. P.; O'Connor, P. B.; McLafferty, F. W. Proc Natl Acad Sci USA $1995,92,2451-2454$.

105. Yu, S. J.; Holliman, C. L.; Remple, D. L.; Gross, M. L. J Am Chem Soc1993, 115, 9676-9682.

106. Zhang, Z. Q.; Marshall, A. G. J Am Soc Mass Spectrom 1998, $9,225-233$

107. Zubarev, R. A.; Kelleher, N. L.; McLafferty, F. W. J Am Chem Soc 1998, 120, 3265-3266. 